\author{
BANACH CENTER PUBLICATIONS, VOLUME ** \\ INSTITUTE OF MATHEMATICS \\ POLISH ACADEMY OF SCIENCES \\ WARSZAWA 201*
}

\title{
ANTIUNITARY REPRESENTATIONS AND MODULAR THEORY
}

\author{
KARL-HERMANN NEEB \\ Department Mathematik, Universität Erlangen-Nürnberg \\ Cauerstrasse 11, 91058 Erlangen, Germany \\ E-mail: neeb@math.fau.de \\ GESTUR ÓLAFSSON \\ Department of mathematics, Louisiana State University \\ Baton Rouge, LA 70803, USA \\ E-mail: olafsson@math.lsu.edu
}

\begin{abstract}
Antiunitary representations of Lie groups take values in the group of unitary and antiunitary operators on a Hilbert space $\mathcal{H}$. In quantum physics, antiunitary operators implement time inversion or a PCT symmetry, and in the modular theory of operator algebras they arise as modular conjugations from cyclic separating vectors of von Neumann algebras. We survey some of the key concepts at the borderline between the theory of local observables (Quantum Field Theory (QFT) in the sense of Araki-Haag-Kastler) and modular theory of operator algebras from the perspective of antiunitary group representations. Here a central point is to encode modular objects in standard subspaces $V \subseteq \mathcal{H}$ which in turn are in one-to-one correspondence with antiunitary representations of the multiplicative group $\mathbb{R}^{\times}$. Half-sided modular inclusions and modular intersections of standard subspaces correspond to antiunitary representations of $\operatorname{Aff}(\mathbb{R})$, and these provide the basic building blocks for a general theory started in the 90s with the ground breaking work of Borchers and Wiesbrock and developed in various directions in the QFT context. The emphasis of these notes lies on the translation between configurations of standard subspaces as they arise in the context of modular localization developed by Brunetti, Guido and Longo, and the more classical context of von Neumann algebras with cyclic separating vectors. Our main point is that configurations of standard subspaces can be studied from the perspective of antiunitary Lie group representations and the geometry of the corresponding spaces, which are often fiber bundles over ordered symmetric spaces. We expect this perspective to provide
\end{abstract}

2010 Mathematics Subject Classification: Primary 22E45; Secondary 81R05, 81T05.

Key words and phrases: antiunitary representation, modular operator, modular conjugation, von Neumann algebra, local observables, half-sided modular inclusion quantum field theory The paper is in final form and no version of it will be published elsewhere. 
new and systematic insight into the much richer configurations of nets of local observables in QFT.

\section{Contents}

1. Introduction . . . . . . . . . . . . . . . . . . 3

2. Antiunitary representations .................... 7

2.1. Involutive group pairs . . . . . . . . . . . . . . . 8

2.2. Extending unitary representations . . . . . . . . . . . . . 10

2.3. One-parameter groups . . . . . . . . . . . . . . . 15

2.4. Some low-dimensional groups . . . . . . . . . . . . . . . 18

3. Modular objects and standard subspaces . . . . . . . . . . . . . 22

3.1. Standard subspaces . . . . . . . . . . . . . . . 22

3.2. Symplectic aspects of standard subspaces . . . . . . . . . . . . 24

3.3. Orthogonal real one-parameter groups . . . . . . . . . . . . . . 25

3.4. Half-sided modular inclusions of standard subspaces . . . . . . . . . . . 26

3.5. Half-sided modular intersections . . . . . . . . . . . . . . . . 31

4. A glimpse of modular theory . . . . . . . . . . . . . . . . . 34

4.1. The Tomita-Takesaki Theorem . . . . . . . . . . . . . . 34

4.2. Translating between standard subspaces and von Neumann algebras . . . 36

4.3. Borchers triples . . . . . . . . . . . . . . . . . . 39

4.4. Modular geometry . . . . . . . . . . . . . . . . 43

5. Nets of standard subspaces and von Neumann algebras . . . . . . . . . . . . 46

5.1. Nets of standard subspaces . . . . . . . . . . . . . . . . 47

5.2. Nets of von Neumann algebras . . . . . . . . . . . . . . . 52

6. Second quantization and modular localization . . . . . . . . . . . . . 55

6.1. Bosonic Fock space . . . . . . . . . . . . . . . . . 55

6.2. Fermionic Fock space . . . . . . . . . . . . . . . 57

6.3. From antiunitary representations to local nets . . . . . . . . . . 60

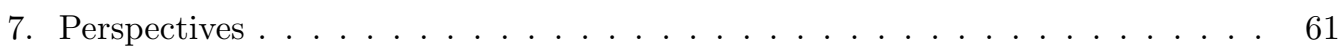

7.1. The Virasoro group . . . . . . . . . . . . . . . . . 61 61

7.2. Euclidean Jordan algebras . . . . . . . . . . . . . . . . . . 61 61

7.3. Hermitian groups . . . . . . . . . . . . . . . . . . 61

7.4. Analytic extension . . . . . . . . . . . . . . . . 62 62

7.5. Geometric standard subspaces . . . . . . . . . . . . . 62 62

7.6. Dual pairs in the Heisenberg group . . . . . . . . . . . . . . . . 63

7.7. A representation theoretic perspective on modular localization . . . . . . 64

A. Appendices .......................... 64

A.1. A lemma on von Neumann algebras . . . . . . . . . . . . . . 64

A.2. Two lemmas on groups . . . . . . . . . . . . . . . . . 65

A.3. Symmetric spaces . . . . . . . . . . . . . . . 65 
1. Introduction. One of the core ideas of quantum theory is that the states of a quantum system correspond to one-dimensional subspaces of a complex Hilbert space $\mathcal{H}$, i.e., the elements $[v]=\mathbb{C} v, v \neq 0$, of its projective space $\mathbb{P}(\mathcal{H})$. This set carries a geometric structure defined by the transition probability

$$
\tau([v],[w]):=\frac{|\langle v, w\rangle|^{2}}{\langle v, v\rangle\langle w, w\rangle} \in[0,1]
$$

between two states $[v]$ and $[w]$, where $d([v],[w])=\arccos \sqrt{\tau([v],[w])} \in[0, \pi / 2]$ is the corresponding Riemannian metric (the Fubini-Study metric), turning it into a RiemannHilbert manifold. Wigner's Theorem characterizes the automorphisms of $(\mathbb{P}(\mathcal{H}), \tau)$, resp., the isometries for the metric, as those bijections induced on $\mathbb{P}(\mathcal{H})$ by elements of the antiunitary group $\mathrm{AU}(\mathcal{H})$ of all linear and antilinear surjective isometries of $\mathcal{H}([\mathrm{Ba64}])$. Accordingly, we have an isomorphism

$$
\operatorname{Aut}(\mathbb{P}(\mathcal{H}), \tau) \cong \operatorname{AU}(\mathcal{H}) / \mathbb{T} \mathbf{1}=: \operatorname{PAU}(\mathcal{H})
$$

of $\operatorname{Aut}(\mathbb{P}(\mathcal{H}), \tau)$ with the projective antiunitary group $\operatorname{PAU}(\mathcal{H})$. So any action of a group $G$ by symmetries of a quantum system leads to a homomorphism $\bar{\pi}: G \rightarrow \operatorname{PAU}(\mathcal{H})$ and further to a homomorphism of the pullback extension $G^{\sharp}=\bar{\pi}^{*} \mathrm{AU}(\mathcal{H})$ of $G$ by the circle group $\mathbb{T}$ to $\mathrm{AU}(\mathcal{H})$, i.e., an antiunitary representation. More precisely, for a pair $\left(G, G_{1}\right)$, where $G_{1} \subseteq G$ is a subgroup of index 2 , a homomorphism $U: G \rightarrow \operatorname{AU}(\mathcal{H})$ is called an antiunitary representation of $\left(G, G_{1}\right)$ if $U_{g}$ is antiunitary for $g \notin G_{1}$. If $G$ is a topological group with two connected components, then we obtain a canonical group pair by $G_{1}:=G_{0}$ (the identity component). In this case an antiunitary representation of $G$ is a continuous homomorphism $U: G \rightarrow \operatorname{AU}(\mathcal{H})$ mapping $G \backslash G_{0}$ into antiunitary operators.

In the mathematical literature on representations, antiunitary operators have never been in the focus, whereas in quantum physics one is forced to consider antiunitary operators to implement a time-reversal symmetry (|Wig59 ). If the dynamics of a quantum system is described by a unitary one-parameter group $U_{t}=e^{i t H}$, where the Hamiltonian $H$ is unbounded and bounded from below, then a unitary time reversal operator $\mathcal{T}$ would lead to the relation $\mathcal{T} H \mathcal{T}=-H$, which is incompatible with $H$ being bounded from below. This problem is overcome by implementing time reversal by an antiunitary operator because it imposes no restrictions on the spectrum of the Hamiltonian. In particular, the PCT Theorem in Quantum Field Theory (QFT) which concerns the implementation of a symmetry reversing parity $(\mathrm{P})$, charge $(\mathrm{C})$ and time $(\mathrm{T})$, leads to an extension of a unitary representation of the Poincaré group $P(d)_{+}^{\uparrow}=\mathbb{R}^{d} \rtimes \mathrm{SO}_{1, d-1}(\mathbb{R})^{\uparrow}$ to an antiunitary representation of the larger group $P(d)_{+} \cong \mathbb{R}^{d} \rtimes \mathrm{SO}_{1, d-1}(\mathbb{R})([$ Ha96, Thm. II.5.1.4]).

In the modular theory of operator algebras one studies pairs $(\mathcal{M}, \Omega)$ consisting of a von Neumann algebra $\mathcal{M} \subseteq B(\mathcal{H})$ and a cyclic separating unit vector $\Omega \in \mathcal{H}$. Then $S(M \Omega):=M^{*} \Omega$ for $M \in \mathcal{M}$ defines an unbounded antilinear involution, and the polar decomposition of its closure $\bar{S}=J \Delta^{1 / 2}$ leads to a positive selfadjoint operator $\Delta=S^{*} \bar{S}$, an antiunitary involution $J$ satisfying the modular relation $J \Delta J=\Delta^{-1}$, and $\alpha_{t}(M):=$ $\Delta^{i t} M \Delta^{-i t}$ defines automorphisms of $\mathcal{M}$ (see [BR87] and 44.1). In particular, we are naturally led to antiunitary symmetries. We say that $(\Delta, J)$ is a pairs of modular objects 
if $J$ is a conjugation and $\Delta$ a positive selfadjoint operator satisfying the modular relation.

To connect this with QFT, we recall the notion of a Haag-Kastler net of $C^{*}$-subalgebras $\mathcal{A}(\mathcal{O})$ of a $C^{*}$-algebra $\mathcal{A}$, associated to (bounded) regions $\mathcal{O}$ in $d$-dimensional Minkowski space. The algebra $\mathcal{A}(\mathcal{O})$ is interpreted as observables that can be measured in the "laboratory" $\mathcal{O}$. Accordingly, one requires isotony, i.e., that $\mathcal{O}_{1} \subseteq \mathcal{O}_{2}$ implies $\mathcal{A}\left(\mathcal{O}_{1}\right) \subseteq$ $\mathcal{A}\left(\mathcal{O}_{2}\right)$ and that the $\mathcal{A}(\mathcal{O})$ generate $\mathcal{A}$. Causality enters by the locality assumption that $\mathcal{A}\left(\mathcal{O}_{1}\right)$ and $\mathcal{A}\left(\mathcal{O}_{2}\right)$ commute if $\mathcal{O}_{1}$ and $\mathcal{O}_{2}$ are space-like separated, i.e., cannot correspond with each other (cf. Example 5.4). Finally one assumes an action $\sigma: P(d)_{+}^{\uparrow} \rightarrow \operatorname{Aut}(\mathcal{A})$ of the connected Poincaré group such that $\sigma_{g}(\mathcal{A}(\mathcal{O}))=\mathcal{A}(g \mathcal{O})$. Every Poincaré invariant state $\omega$ of the algebra $\mathcal{A}$ now leads by the GNS construction to a covariant representation $\left(\pi_{\omega}, \mathcal{H}_{\omega}, \Omega\right)$ of $\mathcal{A}$, and hence to a net $\mathcal{M}(\mathcal{O}):=\pi_{\omega}(\mathcal{A}(\mathcal{O}))^{\prime \prime}$ of von Neumann algebras on $\mathcal{H}_{\omega}$. Whenever $\Omega$ is cyclic and separating for $\mathcal{M}(\mathcal{O})$, we obtain modular objects $\left(\Delta_{\mathcal{O}}, J_{\mathcal{O}}\right)$. This connection between the Araki-Haag-Kastler theory of local observables and modular theory leads naturally to antiunitary group representations (cf. Section 5).

The starting point for the recent development that led to fruitful applications of modular theory in QFT was the Bisognano-Wichmann Theorem, asserting that, the modular automorphisms $\alpha_{t}(M)=\Delta^{i t} M \Delta^{-i t}$ corresponding to the algebra $\mathcal{M}(W)$ of observables corresponding to a wedge domain $W$ in Minkowski space (cf. Definition 4.11) are implemented by the unitary action of a one-parameter group of Lorentz boosts preserving $W$ ([BW76] $)$. This geometric implementation of modular automorphisms in terms of Poincaré transformations was an important first step in a rich development based on the work of Borchers and Wiesbrock in the 1990s Bo92, Bo95, Bo97, Wi92, Wi93, Wi93c. They managed to distill the abstract essence from the Bisognano-Wichmann Theorem which led to a better understanding of the basic configurations of von Neumann algebras in terms of half-sided modular inclusions and modular intersections. This immediately led to very tight connections between the geometry of homogeneous spaces and modular theory BGL93. In his survey Bo00, Borchers described how these concepts have revolutionized quantum field theory. Subsequent developments can be found in [Tr97, Sch97, Ar99, BGL02, Lo08, JM17]; for the approach to Quantum Gravity based on Non-commutative Geometry and Tomita-Takesaki Theory, see in particular [BCL10.

A key insight that simplifies matters considerably is that modular objects $(\Delta, J)$ associated to a pair $(\mathcal{M}, \Omega)$ of a von Neumann algebra $\mathcal{M}$ and a cyclic separating vector $\Omega$ are completely determined by the real subspace

$$
V_{\mathcal{M}}:=\overline{\mathcal{M}_{h} \Omega}, \quad \text { where } \quad \mathcal{M}_{h}=\left\{M \in \mathcal{M}: M^{*}=M\right\} .
$$

It satisfies $V_{\mathcal{M}} \cap i V_{\mathcal{M}}=\{0\}$ and $V_{\mathcal{M}}+i V_{\mathcal{M}}$ is dense in $\mathcal{H}$. Closed real subspaces $V \subseteq \mathcal{H}$ with these two properties are called standard. Every standard subspace $V$ determines by the polar decomposition of the closed operator $S_{V}$ defined on $V+i V$ by $S_{V}(x+i y)=x-i y$ a pair $\left(\Delta_{V}, J_{V}\right)$ of modular objects and, conversely, any such pair $(\Delta, J)$ determines a standard subspace as the fixed point space of $J \Delta^{1 / 2}$ (see Section [3). We refer to [Lo8] for an excellent survey on this correspondence. In QFT, standard subspaces provide the basis for the technique of modular localization, developed by Brunetti, Guido and Longo in [BGL02]. For some applications we refer to [Sch97, MSY06, Sch06, LW11, Ta12, LL14, Mo17. 
From the perspective of antiunitary representations, standard subspaces $V$ with modular objects $(\Delta, J)$ are in one-to-one correspondence with antiunitary representations

$$
U: \mathbb{R}^{\times} \rightarrow \mathrm{AU}(\mathcal{H}) \quad \text { by } \quad U_{-1}=J \quad \text { and } \quad U_{e^{t}}=\Delta^{-i t / 2 \pi}
$$

(Proposition 3.2). Accordingly, antiunitary representations $(U, \mathcal{H})$ of the affine group $\mathrm{Aff}(\mathbb{R}) \cong \mathbb{R} \rtimes \mathbb{R}^{\times}$correspond to one-parameter families of standard subspaces $\left(V_{x}\right)_{x \in \mathbb{R}}$, where $V_{x}$ corresponds to the affine stabilizer group of $x$. Borchers' key insight was that the positive energy condition on the representation of the translation group is intimately related to inclusions of these subspaces. More precisely, $U_{(t, 1)}=e^{i t P}$ satisfies $P \geq 0$ if and only if $U_{(t, 1)} V_{0} \subseteq V_{0}$ holds for all $t \geq 0$ (93.4). This leads to Borchers pairs $(V, U)$ of a standard subspace $V$ and a unitary one-parameter group $\left(U_{t}\right)_{t \in \mathbb{R}}$, a concept that is equivalent to the so-called half-sided modular inclusions $V_{1} \subseteq V_{2}$ of pairs of standard subspaces, which was condensed from the corresponding concept of a half-sided modular inclusion of von Neumann algebras ( $\$ 3.44 .2$ ).

The main objective of this article is to describe certain structures arising in QFT, such as nets of von Neumann algebras and standard subspaces, from the perspective of antiunitary group representations. Since any standard subspace $V$ corresponds to a representation of $\mathbb{R}^{\times}$and inclusions of standard subspaces correspond to antiunitary positive energy representations of $\operatorname{Aff}(\mathbb{R})$, it is very likely that a better understanding of antiunitary representations and corresponding families of standard subspaces provides new insight into the geometric structures underlying QFT. This article is written from a mathematical perspective and we are rather brief on the concrete physical aspects mentioned in \$5.2. We tried to describe the mathematical side of the theory as clearly as possible to make it easier for mathematicians to understand the relevant aspects without going to much into physics. For more details of the physical side, we recommend BDFS00, BGL02, Lo08, LL14. In particular, the programs outlined by Borchers and Wiesbrock, see f.i., Bo97, Bo00, Wi93c, leave much potential for an analysis from the representation theoretic perspective.

The structure of this paper is as follows. In Section 2 we discuss antiunitary representations of group pairs $\left(G, G_{1}\right)$ and criteria for a unitary representation of $G_{1}$ to extend to an antiunitary representation of $G$. An interesting simplifying feature is that, whenever antiunitary extensions exist, they are unique up to equivalence (Theorem 2.11). We show that irreducible unitary representations of $G_{1}$ fall into three types (real, complex and quaternionic) with respect to their extendability behavior to antiunitary representations of $G$. We also take a closer look at antiunitary representations of one-dimensional Lie groups $(2.3)$. Here $\mathbb{R}^{\times}$plays a central role because its antiunitary representations encode modular objects $(\Delta, J)$ as in (1). We conclude Section 2 with a discussion of antiunitary representations of the affine group $\operatorname{Aff}(\mathbb{R})$, the projective group $\mathrm{PGL}_{2}(\mathbb{R})$ and the 3-dimensional Heisenberg group $\operatorname{Heis}\left(\mathbb{R}^{2}\right)$.

Section 3 is devoted to various aspects of standard subspaces as a geometric counterpart of antiunitary representations of $\mathbb{R}^{\times}$. In particular, we discuss how the embedding $V \subseteq \mathcal{H}$ can be obtained from the orthogonal one-parameter group $\left.\Delta^{i t}\right|_{V}$ on $V(\$ 3.3)$, and in $\$ 3.4$ we discuss half-sided modular inclusions of standard subspaces and how they are 
related to antiunitary representations of $\operatorname{Aff}(\mathbb{R}), P(2)_{+}$and $\mathrm{PGL}_{2}(\mathbb{R})$.

In Section 4 we first recall some of the key features of Tomita-Takesaki Theory. $₫ 4.2$ is of key importance because it is devoted to the translation between pairs $(\mathcal{M}, \Omega)$ of von Neumann algebras with cyclic separating vectors and standard subspaces $V$. We have already seen how to obtain a standard subspace $V_{\mathcal{M}}=\overline{\mathcal{M}_{h} \Omega}$ from $(\mathcal{M}, \Omega)$. Conversely, one can use Second Quantization (see Section 6 for details) to associate to each standard subspace $V \subseteq \mathcal{H}$ pairs $\left(\mathcal{R}_{ \pm}(V), \Omega\right)$, where $\mathcal{R}_{ \pm}(V)$ is a von Neumann algebra on the (bosonic/fermionic) Fock space $\mathcal{F}_{ \pm}(\mathcal{H})$. This method has been invented and studied thoroughly by Araki and Woods in the 1960s and 1970s in the context of free bosonic quantum fields ([Ar64, Ar99, AW63, AW68 $)$; some of the corresponding fermionic results are more recent (cf. EO73], BJL02]) and other statistics (anyons) are discussed in Sch97.

A central point is that these correspondences permit to translate between results on configurations of standard subspaces and configurations of von Neumann algebras with common cyclic vectors. We explain this in detail for half-sided modular inclusions and Borchers pairs ( $\$ \$ 4.2$ and 4.3) but we expect it to go much deeper. Keeping in mind that standard subspaces are in one-to-one correspondence with antiunitary representations of $\mathbb{R}^{\times}$and half-sided modular inclusions with antiunitary positive energy representations of $\operatorname{Aff}(\mathbb{R})$, we expect that many interesting results on von Neumann algebras can be obtained from a better understanding of antiunitary representations of Lie group pairs $\left(G, G_{1}\right)$ and configurations of homomorphisms $\gamma:\left(\mathbb{R}^{\times}, \mathbb{R}_{+}^{\times}\right) \rightarrow\left(G, G_{1}\right)$. The construction of free fields by second quantization associates to an antiunitary representation $(U, \mathcal{H})$ of $G$ on the one-particle spaces $\mathcal{H}$, resp., to the corresponding standard subspaces $V_{\gamma}$, a net of Neumann algebras on Fock space. However, there is also a converse aspect which is probably more important, namely that the passage from pairs $(\mathcal{M}, \Omega)$ to the standard subspaces $V_{\mathcal{M}}$ is not restricted to free fields and can be used to attach geometric structure to nets of von Neumann algebras, all encoded in the subgroup of $\mathrm{AU}(\mathcal{H})$ generated by all operators $\Delta_{\mathcal{M}}^{i t}$ and $J_{\mathcal{M}}$. To substantiate this remark, we discuss in Section 5 several aspects of nets of standard subspaces and von Neumann algebras as they arise in QFT. In particular, we consider nets of standard subspaces $\left(V_{\ell}\right)_{\ell \in L}$ arising from antiunitary representations $(U, \mathcal{H})$, which leads to the covariance relation $U_{g} V_{\ell}=V_{g . \ell}$ for $g \in G_{1}$, and one expects geometric information to be encoded in the $G$-action on the index set $L$. A common feature of the natural examples is that $L$ has a fibration over a symmetric space that corresponds to the projection $\left(\Delta_{\ell}, J_{\ell}\right) \mapsto J_{\ell}$, forgetting the modular operator. For details we refer to the discussion of several examples in Section 5. Typical index sets $L$ arise as conjugation orbits $\left\{\gamma^{g},\left(\gamma^{\vee}\right)^{g}: g \in G\right\} \subseteq \operatorname{Hom}\left(\mathbb{R}^{\times}, G\right)$, where $\gamma^{g}(t)=g \gamma(t) g^{-1}$ and $\gamma^{\vee}(t)=\gamma\left(t^{-1}\right)$. In this picture, the above projection simply corresponds to the evaluation map ev-1: $\operatorname{Hom}\left(\mathbb{R}^{\times}, G\right) \rightarrow \operatorname{Inv}(G)$ and the set $\operatorname{Inv}(G)$ of involutions of $G$ is a symmetric space ([Lo69]; Appendix A.3). In many concrete situations, the centralizer of $\gamma(-1)$ in $G$ coincides with the centralizer of the whole subgroup $\gamma\left(\mathbb{R}^{\times}\right)$, so that the conjugacy class $C_{\gamma}=\left\{\gamma^{g}: g \in G\right\}$ can be identified with the conjugacy class $C_{\gamma(-1)}$ of the involution $\gamma(-1)$, and this manifold is a symmetric space. We are therefore led to index sets which are ordered symmetric spaces, and these objects have been studied in 
detail in the 90s. We refer to the monograph HÓ96 for a detailed exposition of their structure theory.

Section 6 presents the second quantization process from standard subspaces $V \subseteq \mathcal{H}$ to pairs $\left(\mathcal{R}^{ \pm}(V), \Omega\right)$ in a uniform way, stressing in particular the similarity between the bosonic and the fermionic case.

In the final Section 7 we briefly describe some perspectives and open problems. Antiunitary representations occur naturally for interesting classes of groups such as the Virasoro group, conformal and affine groups related to euclidean Jordan algebras, and automorphism groups of bounded symmetric domains. For detailed results we refer to the forthcoming paper NÓ17. In \$7.6 we also explain how second quantization leads to interesting dual pairs in the Heisenberg group Heis $(\mathcal{H})$ : Any standard subspace $V \subseteq \mathcal{H}$ satisfying the factoriality condition $V \cap V^{\prime}=\{0\}$, where $V^{\prime}$ is the symplectic orthogonal space, leads by restriction of the irreducible Fock representation of Heis $(\mathcal{H})$ to a factor representation of the subgroup Heis $(V)$, which forms a dual pair with Heis $\left(V^{\prime}\right)$ in Heis $(\mathcal{H})$ (both subgroups are their mutual centralizers). So far, such dual pairs have not been exploited systematically from the perspective of unitary representations of infinite dimensional Lie groups.

Some basic auxiliary lemmas and definitions have been collected in the appendix.

Notation and conventions: As customary in physics, the scalar product $\langle\cdot, \cdot\rangle$ on a complex Hilbert space $\mathcal{H}$ is linear in the second argument.

$\llbracket S \rrbracket$ denotes the closed subspace of a Hilbert space $\mathcal{H}$ generated by the subset $S$. $\{a, b\}:=a b+b a$ is the anti-commutator of two elements of an associative algebra. For the cyclic group of order $n$ we write $\mathbb{Z}_{n}=\mathbb{Z} / n \mathbb{Z}$.

For $\mathbf{x}, \mathbf{y} \in \mathbb{R}^{d-1}$, we write $\mathbf{x y}=\sum_{j=1}^{d-1} x_{j} y_{j}$ for the scalar product and, for $x=$ $\left(x_{0}, \mathbf{x}\right) \in \mathbb{R}^{d}$, we write $[x, y]=x_{0} y_{0}-\mathbf{x y}$ for the Lorentzian scalar product on the $d$ dimensional Minkowski space $\mathbb{R}^{1, d-1} \cong \mathbb{R}^{d}$. The light cone in Minkowski space is denoted

$$
V_{+}=\left\{x \in \mathbb{R}^{1, d-1}: x_{0}>0,[x, x]>0\right\} .
$$

Here is our notation for some of the groups arising in physics:

- the Poincaré group $P(d) \cong \mathbb{R}^{1, d-1} \rtimes \mathrm{O}_{1, d-1}(\mathbb{R})$ of affine isometries of $\mathbb{R}^{1, d-1}$,

- $P(d)_{+}=\mathbb{R}^{1, d-1} \rtimes \mathrm{SO}_{1, d-1}(\mathbb{R})$ is the subgroup of orientation preserving maps, and

- $P(d)^{\uparrow}=\mathbb{R}^{1, d-1} \rtimes \mathrm{O}_{1, d-1}(\mathbb{R})^{\uparrow}$ with $\mathrm{O}_{1, d-1}(\mathbb{R})^{\uparrow}=\left\{g \in \mathrm{O}_{1, d-1}(\mathbb{R}): g V_{+}=V_{+}\right\}$the subgroup preserving the causal structure.

- The corresponding conformal group is $\mathrm{O}_{2, d}(\mathbb{R})$, acting on the conformal compactification $\mathbb{S}^{1} \times \mathbb{S}^{d-1}$ of $M^{d}$ with the kernel $\{ \pm \mathbf{1}\}$ (see [HN12, $\left.\S 17.4\right]$ ).

If not otherwise states, all Lie groups in this paper are finite dimensional.

2. Antiunitary representations. In this section we discuss antiunitary representations of group pairs $\left(G, G_{1}\right)$ and criteria for a unitary representation of $G_{1}$ to extend to an antiunitary representation of $G$. We start in $\$ 2.1$ with some general remarks on group pairs $\left(G, G_{1}\right)$ and how to classify twists in this context. We also take a closer look 
at antiunitary representations of one-dimensional Lie groups in $\$ 2.3$ and discuss antiunitary representations of the affine group $\operatorname{Aff}(\mathbb{R})$, the projective group $\mathrm{PGL}_{2}(\mathbb{R})$ and the 3 -dimensional Heisenberg group in $\$ 2.4$

Definition 2.1. An antiunitary representation $(U, \mathcal{H})$ of a group pair $\left(G, G_{1}\right)$, where $G_{1} \subseteq G$ is a subgroup of index 2 , is a homomorphism $U$ of $G$ into the group $\operatorname{AU}(\mathcal{H})$ of unitary or antiunitary operators on a complex Hilbert space $\mathcal{H}$ for which $G_{1}=U_{G}^{-1}(\mathrm{U}(\mathcal{H}))$, i.e., $G_{1}$ is represented by unitary operators and the coset $G \backslash G_{1}$ by antiunitary operators.

If $G$ is a Lie group, then $\left(G, G_{1}\right)$ is called a Lie group pair.

If $G$ is a topological group with two connected components, then we obtain a canonical group pair by $G_{1}:=G_{0}$ (the identity component). In this case an antiunitary representation of $G$ is a continuous homomorphism $U: G \rightarrow \operatorname{AU}(\mathcal{H})$ mapping $G \backslash G_{0}$ into antiunitary operators.

We start this section with a discussion of the natural class of group pairs that will show up in the context of antiunitary representations.

\subsection{Involutive group pairs.}

Definition 2.2. An involutive group pair is a pair $\left(G, G_{1}\right)$ of groups, where $G_{1} \subseteq G$ is a subgroup of index 2 and there exists an element $g \in G \backslash G_{1}$ with $g^{2} \in Z\left(G_{1}\right)$. Then $\tau\left(g_{1}\right):=g g_{1} g^{-1}$ defines an involutive automorphism of $G_{1}$.

In most examples that we encounter below $G$ is a Lie group with two connected components and $G_{1}$ is its identity component.

REMARK 2.3. (a) If $g^{2} \in Z\left(G_{1}\right)$, then other elements $g h \in g G_{1}$ need not have central squares. From $(g h)^{2}=g h g h=g^{2} \tau(h) h$ it follows that $(g h)^{2}$ is central if and only if $\tau(h) h \in Z\left(G_{1}\right)$, which is in particular the case if $\tau(h)=h^{-1}$.

(b) If $G$ is a Lie group, then any conjugacy class $C_{g}$ of $g \in G \backslash G_{1}$ with $g^{2} \in Z(G)$ carries a natural symmetric space structure (Appendix A.3). In fact, the stabilizer of $g$ in $G_{1}$ is $G_{1}^{\tau}$, so that we obtain a diffeomorphism

$$
G_{1} / G_{1}^{\tau} \rightarrow C_{g}, \quad h G_{1}^{\tau} \mapsto h g h^{-1}=h \tau(h)^{-1} g .
$$

EXAmples 2.4. (a) Let $\mathcal{H}$ be a complex Hilbert space and $\left(G, G_{1}\right):=(\mathrm{AU}(\mathcal{H}), \mathrm{U}(\mathcal{H}))$. An antiunitary operator $J \in \mathrm{AU}(\mathcal{H})$ is called a conjugation if $J^{2}=\mathbf{1}$ and an anticonjugation if $J^{2}=\mathbf{- 1}$. Conjugations always exist and define a real structure on $\mathcal{H}$ in the sense that $\mathcal{H}^{J}=\operatorname{Fix}(J):=\operatorname{ker}(J-\mathbf{1})$ is a real Hilbert space whose complexification is $\mathcal{H} \mathbb{1}$ Anticonjugations define on $\mathcal{H}$ a quaternionic structure, hence do not exist if $\mathcal{H}$ is of finite odd dimension.

Any (anti-)conjugation $J$ on $\mathcal{H}$ is contained in $G \backslash G_{1}$ and satisfies $J^{2} \in\{ \pm \mathbf{1}\} \subseteq$ $Z(\mathrm{U}(\mathcal{H}))$.

(b) If $G_{1}$ is a group and $\tau \in \operatorname{Aut}\left(G_{1}\right)$ is an involutive automorphism, then $G:=G_{1} \rtimes\{\mathbf{1}, \tau\}$ defines an involutive group pair.

\footnotetext{
${ }^{1}$ For the existence, fix an orthonormal basis $\left(e_{j}\right)_{j \in I}$ of $\mathcal{H}$ and defined $J$ to be antilinear with $J e_{j}=e_{j}$ for every $j \in I$.
} 
ExAmPle 2.5. (A non-involutive group pair) Let $\sigma: C_{4} \rightarrow \operatorname{Aut}(\mathbb{C})$ denote the natural action of the subgroup $C_{4}=\{ \pm 1, \pm i\} \subseteq \mathbb{T}$ by multiplication and form the semidirect product group $G:=\mathbb{C} \rtimes_{\sigma} C_{4}$. Then $G_{1}:=\mathbb{C} \rtimes_{\sigma}\{ \pm 1\}$ is a subgroup of index 2 but no element $g \in G \backslash G_{1}$ satisfies $g^{2} \in Z\left(G_{1}\right)$ because $g^{2}$ acts on $\mathbb{C}$ as $-\operatorname{id}_{\mathbb{C}}$.

REMARK 2.6. (Classification of involutive group pairs) (a) Suppose we are given a group $G$ and an involutive automorphism $\tau$ of $G$. We want to classify all group extensions

$$
\mathbf{1} \rightarrow G \rightarrow G^{\sharp} \rightarrow \mathbb{Z}_{2} \rightarrow \mathbf{1},
$$

where the corresponding involution in the group $\operatorname{Out}(G)$ of outer automorphisms of $G$ is represented by $\tau$. In view of HN12, Thm. 18.1.13], the equivalence classes of these extensions are parametrized by the cohomology group $H_{\tau}^{2}\left(\mathbb{Z}_{2}, Z(G)\right)$, where $\overline{1}$ acts on $Z(G)$ by $\left.\tau\right|_{Z(G)}$. As any cocycle $f: \mathbb{Z}_{2} \times \mathbb{Z}_{2} \rightarrow Z(G)$ normalized by $f(\overline{0}, g)=f(g, \overline{0})=e$ is determined by the element $z:=f(\overline{1}, \overline{1}) \in Z(G)$ because all other values vanish, the group structure on the corresponding extension is given by an element $\widehat{\tau} \in G^{\sharp} \backslash G$ satisfying

$$
\widehat{\tau}^{2}=z \quad \text { and } \quad \widehat{\tau} g \widehat{\tau}^{-1}=\tau(g) \quad \text { for } \quad g \in G .
$$

This description shows in particular that $\tau(z)=z$, and a closer inspection of the cohomology groups yields

$$
H_{\tau}^{2}\left(\mathbb{Z}_{2}, Z(G)\right) \cong Z(G)^{\tau} / Z(G)_{\tau}, \quad \text { where } \quad Z(G)_{\tau}:=\{\tau(z) z: z \in Z(G)\}
$$

([HN12, Ex. 18.3.5]).

(b) For $\left.\tau\right|_{Z(G)}=\operatorname{id}_{Z(G)}$ we have

$$
Z(G)_{\tau}=\left\{z^{2}: z \in Z(G)\right\} \quad \text { and } \quad H_{\tau}^{2}\left(\mathbb{Z}_{2}, Z(G)\right) \cong Z(G) / Z(G)_{\tau} .
$$

(c) For $\left.\tau\right|_{Z(G)}=-\operatorname{id}_{Z(G)}$, we have

$$
H_{\tau}^{2}\left(\mathbb{Z}_{2}, Z(G)\right) \cong Z(G)^{\tau}=\left\{z \in Z(G): z^{2}=e\right\},
$$

the subgroup of central involutions.

REMARK 2.7. Although by (2) the cohomology groups $H_{\tau}^{2}\left(\mathbb{Z}_{2}, Z(G)\right)$ are elementary abelian two groups, one cannot expect any bound on the order of an element $g \in G^{\sharp} \backslash G$. In the cyclic group $G^{\sharp}=\mathbb{Z}_{2^{n}}$ with $G \cong \mathbb{Z}_{2^{n-1}}$, any element of $G^{\sharp} \backslash G$ is of order $2^{n}$.

ExAmPLE 2.8. (a) For $G=\mathbb{R}$, Remark 2.6 implies that $H_{\tau}^{2}\left(\mathbb{Z}_{2}, \mathbb{R}\right)=\{0\}$ for any involutive automorphism $\tau$. This implies that $G^{\sharp} \cong \mathbb{R} \rtimes_{\tau} \mathbb{Z}_{2}$.

(b) For $G=\mathbb{T}$, the cohomology is trivial for $\tau=\mathrm{id}_{\mathbb{T}}$, but for $\tau(z)=z^{-1}$ the group

$$
H_{\tau}^{2}\left(\mathbb{Z}_{2}, \mathbb{T}\right) \cong\left\{z \in \mathbb{T}: z^{2}=1\right\}=\{ \pm 1\}
$$

is non-trivial. A concrete model for the non-trivial extension with $\widehat{\tau}^{2}=-1$ is given by the subgroup

$$
\operatorname{Pin}_{2}(\mathbb{R})=\exp (\mathbb{R} I) \cup J \exp (\mathbb{R} I) \subseteq \mathbb{H}^{\times},
$$

where $I$ and $J$ are the two generators of the skew-field $\mathbb{H}$ of quaternions satisfying $I^{2}=$ $J^{2}=-\mathbf{1}$ and $I J=-J I$ ([HN12, Ex. B.3.24]). This is a 1-dimensional Lie group without a simply connected covering group ([ $\mathrm{HN12}$, Ex. 18.2.4])

EXAMPLES 2.9. Here are some concrete involutive group pairs $\left(G, G_{1}\right)$ that we shall be dealing with. 
(a) $G=\operatorname{Aff}(\mathbb{R}) \cong \mathbb{R} \rtimes \mathbb{R}^{\times}$with $G_{1} \cong \mathbb{R} \rtimes \mathbb{R}_{+}^{\times}$, the identity component. Here $r_{x}^{2}=\mathbf{1}$ holds for the reflections $r_{x}=(2 x,-1)$ in $x \in \mathbb{R}$.

(b) The automorphism group $G=\mathrm{PGL}_{2}(\mathbb{R})$ of the real projective line $\mathbb{P}_{1}(\mathbb{R}) \cong$ $\mathbb{R} \cup\{\infty\}$, where $G_{1}=\mathrm{PSL}_{2}(\mathbb{R})$ is the identity component and reflections in $\mathrm{GL}_{2}(\mathbb{R})$ lead to orientation reversing involutions of $\mathbb{S}^{1}$.

(c) The Poincaré group $P(d)=\mathbb{R}^{1, d-1} \rtimes \mathrm{O}_{1, d-1}(\mathbb{R})$ of $d$-dimensional Minkowski space $\mathbb{R}^{1, d-1}$ contains the subgroup $P(d)_{+}=\mathbb{R}^{1, d-1} \rtimes \mathrm{SO}_{1, d-1}(\mathbb{R})$ of orientation preserving affine isometries. Then we obtain the involutive group pair $\left(G, G_{1}\right)$ with $G:=P(d)_{+}$and $G_{1}:=P(d)_{+}^{\uparrow}$. In the following the involution $R_{01}:=\operatorname{diag}(-1,-1,1, \ldots, 1) \in G \backslash G_{1}$ plays an important role (cf. Lemma 4.12).

(d) For a (bounded) symmetric domain $\mathcal{D} \subseteq \mathbb{C}^{n}$, the group $\operatorname{Aut}(\mathcal{D})$ of biholomorphic automorphisms is an index 2-subgroup of the hermitian group $\operatorname{AAut}(\mathcal{D})$ of all bijections of $\mathcal{D}$ that are either holomorphic or antiholomorphic. There always exist antiholomorphic involutions $\sigma$ in $\operatorname{AAut}(\mathcal{D})$ (see [Ka97] for a classification covering even the infinite dimensional case). For any such involution $\sigma$, we obtain by $G_{1}:=\operatorname{Aut}(\mathcal{D})_{0}$ and $G:=G_{1} \rtimes\{\mathbf{1}, \sigma\}$ an involutive group pair (cf. [NÓ17] and \$7.3).

2.2. Extending unitary representations. Suppose that $G_{1}$ is an index two subgroup of the group $G$ and $(U, \mathcal{H})$ is a unitary representation of $G_{1}$. In this subsection we discuss extensions of $U$ to antiunitary representations of $G$. In particular, we show that, in analogy to the classical case $G=G_{1} \times \mathbb{Z}_{2}$, irreducible antiunitary representations fall into three types that we call real, complex and quaternionic, according to their commutant.

We start with the following lemma on a situation where extensions always exist because the representation has been doubled in a suitable way.

Lemma 2.10. (Extension Lemma) Let $G_{1} \subseteq G$ be a subgroup of index two and $(U, \mathcal{H})$ be a unitary representation of $G_{1}$. Fix $r \in G \backslash G_{1}$ and consider the automorphism $\tau(g):=$ rgr $^{-1}$ of $G_{1}$. Then the unitary representation $V:=U \oplus U^{*} \circ \tau$ on $\mathcal{H} \oplus \mathcal{H}^{*}$ extends to an antiunitary representation of $G$.

Proof. Let $\Phi: \mathcal{H} \rightarrow \mathcal{H}^{*}, \Phi(v)(w):=\langle v, w\rangle$ denote the canonical antiunitary operator and note that $U_{g}^{*} \circ \Phi=\Phi \circ U_{g}$ for $g \in G_{1}$. We consider the antiunitary operator

$$
J: \mathcal{H} \oplus \mathcal{H}^{*} \rightarrow \mathcal{H} \oplus \mathcal{H}^{*}, \quad J(v, \lambda):=\left(\Phi^{-1} \lambda, \Phi U_{r^{2}} v\right) .
$$

It satisfies

$$
J^{2}(v, \lambda)=J\left(\Phi^{-1} \lambda, \Phi U_{r^{2}} v\right)=\left(U_{r^{2}} v, \Phi U_{r^{2}} \Phi^{-1} \lambda\right)=\left(U_{r^{2}} v, U_{r^{2}}^{*} \lambda\right)=V_{r^{2}}(v, \lambda),
$$

where we have used $\tau\left(r^{2}\right)=r^{2}$ for the last equality. This proves that $J^{2}=V_{r^{2}}$. We now show that $J V_{g} J^{-1}=V_{\tau(g)}$ for $g \in G$ :

$$
\begin{aligned}
J V_{g}(v, \lambda) & =J\left(U_{g} v, U_{\tau(g)}^{*} \lambda\right)=\left(\Phi^{-1} U_{\tau(g)}^{*} \lambda, \Phi U_{r^{2}} U_{g} v\right)=\left(U_{\tau(g)} \Phi^{-1} \lambda, \Phi U_{\tau^{2}(g)} U_{r^{2}} v\right) \\
& =\left(U_{\tau(g)} \Phi^{-1} \lambda, U_{\tau^{2}(g)}^{*} \Phi U_{r^{2}} v\right)=V_{\tau(g)}\left(\Phi^{-1} \lambda, \Phi U_{r^{2}} v\right)=V_{\tau(g)} J(v, \lambda) .
\end{aligned}
$$

The relations $J^{2}=V_{r^{2}}$ and $J V_{g} J^{-1}=V_{\tau(g)}$ now imply by direct calculation that the assignment $V_{g r}:=V_{g} J$ for $g \in G_{1}$ defines an extension of $V$ to an antiunitary representation of $G$ (Lemma A.4). 
The following theorem implies that extensions of unitary representations of $G_{1}$ to antiunitary representations $(U, \mathcal{H})$ of $G$ are always unique up to isomorphism. It also describes the situation for irreducible representations. Note that the commutant

$$
U_{G}^{\prime}=\left\{A \in B(\mathcal{H}):(\forall g \in G) A U_{g}=U_{g} A\right\}
$$

is not a complex subalgebra of $B(\mathcal{H})$ because some $U_{g}$ are antilinear.

THEOREM 2.11. Let $G_{1} \subseteq G$ be a subgroup of index two, $r \in G \backslash G_{1}$ and $\tau(g):=r g r^{-1}$ for $g \in G_{1}$.

(a) For two antiunitary representation $\left(U^{j}, \mathcal{H}_{j}\right)_{j=1,2}$, we then have

$$
\left.\left.U^{1} \cong U^{2} \Longleftrightarrow U^{1}\right|_{G_{1}} \cong U^{2}\right|_{G_{1}}
$$

(b) For any antiunitary representation $(U, \mathcal{H})$ of $\left(G, G_{1}\right)$, the von Neumann algebra $U_{G_{1}}^{\prime}$ is the complexification of the real algebra $U_{G}^{\prime}$.

(c) An antiunitary representation $(U, \mathcal{H})$ of $\left(G, G_{1}\right)$ is irreducible if and only if its commutant $U_{G}^{\prime}$ is isomorphic to $\mathbb{R}, \mathbb{C}$ or $\mathbb{H}$. More specifically:

(i) If $U_{G}^{\prime} \cong \mathbb{R}$, then $U_{G_{1}}^{\prime} \cong \mathbb{C}$ and $\left.U\right|_{G_{1}}$ is irreducible.

(ii) If $U_{G}^{\prime} \cong \mathbb{C}$, then $U_{G_{1}}^{\prime} \cong \mathbb{C}^{2}$ and $\left.U\right|_{G_{1}}$ is a direct sum of two inequivalent irreducible representations which do not extend to an antiunitary representation of $G$.

(iii) If $U_{G}^{\prime} \cong \mathbb{H}$, then $U_{G_{1}}^{\prime} \cong M_{2}(\mathbb{C})$ and $\left.U\right|_{G_{1}}$ is a direct sum of two equivalent irreducible representations which do not extend to an antiunitary representation of $G$.

(d) For an irreducible unitary representation $(U, \mathcal{H})$ of $G_{1}$, either

(i) $U$ extends to an antiunitary representations $\bar{U}$ of $G$, and then $\bar{U}$ is irreducible with $\bar{U}_{G}^{\prime} \cong \mathbb{R} ;$ or

(ii) $U$ does not extend to an antiunitary representation of $G$. Then $V:=U \oplus U^{*} \circ \tau$ extends to an irreducible antiunitary representation of $G$ and $V_{G}^{\prime} \cong \mathbb{C}$ if $U^{*} \circ \tau ¥ U$ and $V_{G}^{\prime} \cong \mathbb{H}$ if $U^{*} \circ \tau \cong U$.

Proof. (a) 2 Let $\Phi: \mathcal{H}_{1} \rightarrow \mathcal{H}_{2}$ be a unitary intertwining operator for the representations $\left.U^{j}\right|_{G_{1}}$. Pick $r \in G \backslash G_{1}$ and consider the antiunitary operators $J_{j}:=U_{r}^{j} \in \operatorname{AU}\left(\mathcal{H}_{j}\right)$. Then the unitary operator $U:=J_{1}^{-1} \Phi^{-1} J_{2} \Phi \in \mathrm{U}\left(\mathcal{H}_{1}\right)$ commutes with $U_{G_{1}}^{1}$. The map $j_{1}(M):=J_{1} M J_{1}^{-1}$ defines an antilinear automorphism of the von Neumann algebra $\left(U_{G_{1}}^{1}\right)^{\prime}$ satisfying

$$
j_{1}(U)=\Phi^{-1} J_{2} \Phi J_{1}^{-1}=\Phi^{-1} J_{2}^{-1} U_{r^{2}}^{2} \Phi J_{1}^{-1}=\Phi^{-1} J_{2}^{-1} \Phi U_{r^{2}}^{1} J_{1}^{-1}=\Phi^{-1} J_{2}^{-1} \Phi J_{1}=U^{-1} .
$$

Therefore Lemma A.1(c) implies the existence of a unitary operator $V \in\left(U_{G_{1}}^{1}\right)^{\prime}$ with $V^{2}=U^{-1}$ and $j_{1}(V)=V^{-1}$. With $\Psi:=\Phi \circ V$, this leads to

$$
\Psi^{-1} J_{2} \Psi=V^{-1} \Phi^{-1} J_{2} \Phi V=V^{-1} J_{1} U V=V^{-1} U^{-1} J_{1} V=V J_{1} V=V V^{-1} J_{1}=J_{1} .
$$

We conclude that the antiunitary representations $U^{1}$ and $U^{2}$ are equivalent.

\footnotetext{
${ }^{2}$ In the finite dimensional context, this was already known to E. Wigner; see Wig59, p. 344].
} 
(b) Let $J:=U_{r}$. Then $U_{G_{1}}^{\prime}$ is invariant under the antilinear automorphism $j(M):=$ $J M J^{-1}$. Since $J^{2}$ commutes with $U_{G_{1}}^{\prime}$, it is involutive. As $U_{G}^{\prime}$ is the set of fixed points of $j$, it is a real form of the complex vector space $U_{G_{1}}^{\prime}$. This implies the assertion.

(c) The closed complex subspaces invariant under $U_{G}$ are precisely the closed real subspaces of the underlying real space $\mathcal{H}^{\mathbb{R}}$ invariant under the group $\mathbb{T} \cdot U_{G}$. Therefore $U_{G}$ is irreducible if and only if the real representation of $\mathbb{T} \cdot U_{G}$ on $\mathcal{H}^{\mathbb{R}}$ is irreducible, which is equivalent to its commutant being isomorphic to $\mathbb{R}, \mathbb{C}$ or $\mathbb{H}$ ([StVa02, Thm. 1]). Next we observe that the real linear commutant of $\mathbb{T} \mathbf{1}$ consists of the complex linear operators. Therefore the real linear commutant of $\mathbb{T} \cdot U_{G}$ equals the complex linear commutant $U_{G}^{\prime}$. Now (b) implies that $U_{G}^{\prime} \cong \mathbb{R}, \mathbb{C}, \mathbb{H}$ leads to $U_{G_{1}}^{\prime} \cong \mathbb{C}, \mathbb{C}^{2}, M_{2}(\mathbb{C})$, respectively.

In the first case $\left.U\right|_{G_{1}}$ is irreducible. In the second case $\mathcal{H} \cong \mathcal{H}_{+} \oplus \mathcal{H}_{-}$, where $\mathcal{H}_{ \pm}$ are $G_{1}$-invariant subspaces on which the $G_{1}$-representations are irreducible and nonequivalent. As $U_{r}$ permutes the $G_{1}$-isotypical subspaces, $U_{r} \mathcal{H}_{ \pm}=\mathcal{H}_{\mp}$. For the representations $U^{ \pm}$of $G_{1}$ on $\mathcal{H}_{ \pm}$, this implies that $U^{-} \cong\left(U^{+}\right)^{*} \circ \tau$. If $U^{+}$or $U^{-}$extends to an antiunitary representation of $G$, then $\left.U\right|_{G_{1}}$ has an extension to a reducible representation $\widetilde{U}$ of $G$. As $U$ is irreducible, this contradicts (a). In the third case we have a similar decomposition with $U^{+} \cong U^{-}$. Again, the irreducibility of $U$, combined with (a), implies that $U^{ \pm}$do not extend to $G$.

(d) If $U$ extends to an antiunitary representation $\bar{U}$ of $G$ on the same space, then this representation is obviously irreducible and (c) implies that $\bar{U}_{G}^{\prime} \cong \mathbb{R}$. If such an extension does not exist, then the Extension Lemma 2.10 provides an extension of $V:=U \oplus U^{*} \circ \tau$ to an antiunitary representation of $G$ by

$$
V_{r}:=J, \quad \text { where } \quad J(v, \lambda):=\left(\Phi^{-1} \lambda, U_{r^{2}}^{*} \Phi v\right) .
$$

If $U^{*} \circ \tau \neq U$, then $V_{G_{1}}^{\prime} \cong \mathbb{C}^{2}$, and if $U^{*} \circ \tau \cong U$, then $V_{G_{1}}^{\prime} \cong M_{2}(\mathbb{C})$.

In the first case the algebra $V_{G_{1}}^{\prime} \cong \mathbb{C}^{2}$ acts by diagonal operators $T_{(a, b)}(v, \lambda):=$ $(a v, b \lambda)$. Such an operator commutes with $J$ if and only if $b=\bar{a}$. Therefore $V_{G}^{\prime} \cong \mathbb{C}$, and thus the representation $V$ of $G$ is irreducible.

In the second case, $V_{G}^{\prime}$ is a real form of $V_{G_{1}}^{\prime} \cong M_{2}(\mathbb{C})$. We show that the representation $\left(V, \mathcal{H} \oplus \mathcal{H}^{*}\right)$ of $G$ is irreducible. If this is not the case, there exists a proper $G$-invariant subspace $\mathcal{K} \subseteq \mathcal{H} \oplus \mathcal{H}^{*}$. As $\left.V\right|_{G_{1}} \cong U \oplus U$, the $G_{1}$-representation on $\mathcal{K}$ must be irreducible and equivalent to $U$. This contradicts the non-extendability of $U$ to an antiunitary representation of $G$. Therefore $V$ is irreducible and $V_{G}^{\prime}$ is isomorphic to $\mathbb{H}$.

Definition 2.12. (Three types of irreducible representations 3 ) We keep the notation of the preceding theorem. If $(U, \mathcal{H})$ is an irreducible unitary representation of $G_{1}$ with $U \cong U^{*} \circ \tau$, then there exists a $\Phi \in \mathrm{AU}(\mathcal{H})$ with $\Phi U_{g} \Phi^{-1}=U_{r g r^{-1}}$ for $g \in G_{1}$. By Schur's Lemma, such an operator $\Phi$ is unique up to a scalar factor in $\mathbb{T}$, so that $\Phi^{2}$ does not depend on the concrete choice of $\Phi$. Therefore an antiunitary extension to $G$ exists if and only if $\Phi^{2}=U_{r^{2}}$. Then we call $(U, \mathcal{H})$ of real type (with respect to $\tau$ ). If this is not the case, but $U \cong U^{*} \circ \tau$, then $(U, \mathcal{H})$ is said to be of quaternionic type (with respect to $\tau$ ), and otherwise we say that it is of complex type (with respect to $\tau$ ). This terminology matches

\footnotetext{
${ }^{3}$ In a special context, this classification by three types can already be found in Wigner's book Wig59, §26, p. 343].
} 
the type of the commutant of the corresponding irreducible antiunitary representation of $G$.

ExAmple 2.13. (a) If $\mathcal{H}=\mathbb{C}$ is one-dimensional, then $\mathrm{AU}(\mathcal{H})=\mathbb{T}\{\mathbf{1}, J\} \cong \mathrm{O}_{2}$ ( $\mathbb{R}$ ) for any conjugation $J$. We conclude in particular that all antiunitary operators are involutions.

(b) If $\mathcal{H}=\mathbb{C}^{2}$ is two-dimensional, we can already see all types of situations for groups generated by a single antiunitary operator, i.e., for antiunitary representations of the pair $\left(G, G_{1}\right)=(\mathbb{Z}, 2 \mathbb{Z})$

Let $J \in \mathrm{AU}(\mathcal{H})$ be antiunitary and $J^{2} \in \mathrm{U}(\mathcal{H})$ be its square. If $J^{2}=\mathbf{1}$, then $J$ is a conjugation, so that there are proper $J$-invariant subspaces. If $J^{2}=-\mathbf{1}$, then $J$ is an anticonjugation defining a quaternionic structure on $\mathbb{C}^{2} \cong \mathbb{H}$. In particular, the representation is irreducible with $U_{G}^{\prime} \cong \mathbb{H}$ and $U_{G_{1}}^{\prime}=B(\mathcal{H}) \cong M_{2}(\mathbb{C})$.

Assume that $J^{4} \neq \mathbf{1}$. Then $J^{2}$ is not an involution, so that it has an eigenvalue $\lambda \neq \pm 1$. If $\mathcal{H}^{\lambda}$ is the corresponding eigenspace, then $J \mathcal{H}^{\lambda}=\mathcal{H}^{\bar{\lambda}}$, so that $\operatorname{Spec}\left(J^{2}\right)=\{\lambda, \bar{\lambda}\}$. Choosing an orthonormal basis $e_{1}, e_{2}$ such that $e_{1} \in \mathcal{H}^{\lambda}$ and $e_{2}:=J e_{1} \in \mathcal{H}^{\bar{\lambda}}$, we obtain $J e_{2}=J^{2} e_{1}=\lambda e_{1}$, so that $J$ is determined up to equivalence. The corresponding representation on $\mathbb{C}^{2}$ is irreducible with $U_{G_{1}}^{\prime} \cong \mathbb{C}^{2}$ and $U_{G}^{\prime} \cong \mathbb{C}$ (Theorem 2.11(c)).

ExAmPLE 2.14. (a) For $G=G_{1} \times \mathbb{Z}_{2}$, the concepts of real/complex/quaternionic type coincides with the classical definition for $G_{1}$, as the characterization in Theorem 2.11] shows.

(b) For $G=G_{1} \rtimes\{\mathbf{1}, \tau\}$ and $\tau^{2}=\operatorname{id}_{G_{1}}$, the extendability of an irreducible unitary representation $(U, \mathcal{H})$ of $G_{1}$ is equivalent to the existence of a conjugation $J \in \operatorname{AU}(\mathcal{H})$ satisfying $J U_{g} J=U_{\tau(g)}$ for $g \in G_{1}$.

If $U \cong U^{*} \circ \tau$, then a $J \in \mathrm{AU}(\mathcal{H})$ satisfying $J U_{g} J=U_{\tau(g)}$ for $g \in G_{1}$ exists and $J^{2} \in U_{G_{1}}^{\prime}=\mathbb{C} \mathbf{1}$, together with $J J^{2} J=J^{2}$ imply $J^{2} \in\{ \pm \mathbf{1}\}$. Accordingly, $U$ is of real, resp., quaternionic type if $J^{2}=\mathbf{1}$, resp., $J^{2}=-\mathbf{1}$.

Example 2.15. (a) For $G=\mathbb{Z}_{2}$ and $G_{1}=\{e\}$, Theorem 2.11)(a) reproduces the fact that all conjugations on $\mathcal{H}$ are conjugate under $\mathrm{U}(\mathcal{H})$.

(b) For $G=\mathbb{Z}_{4}=\mathbb{Z} / 4 \mathbb{Z}$ and $G_{1}=\{\overline{0}, \overline{2}\}$, the case of antiunitary representations with $U_{\overline{2}}=-\mathbf{1}$ likewise implies that all anticonjugations are conjugate under $\mathrm{U}(\mathcal{H})$.

(c) The irreducible unitary representation of $G=\mathrm{SU}_{2}(\mathbb{C})$ on $\mathbb{C}^{2} \cong \mathbb{H}$ (by left multiplication) is of quaternionic type. The complex structure on $\mathbb{H}$ is defined by the right multiplication with $I$. Then $\Phi(a)=a J$ defines a $G$-equivariant anticonjugation on $\mathbb{C}^{2}$. Therefore the representation is of quaternionic type.

(d) For any compact connected Lie group $G_{1}$, the irreducible unitary representations $\left(U_{\lambda}, \mathcal{H}_{\lambda}\right)$ are classified in terms of their highest weights $\lambda$ with respect to a maximal torus $T \subseteq G_{1}$, resp., by the orbits $\mathcal{W} \lambda$ under the Weyl group $\mathcal{W}$. As $-\mathcal{W} \lambda$ is the Weyl group orbit of the dual representation, $U_{\lambda}$ is self-dual if and only if $-\lambda \in \mathcal{W} \lambda$ ( BtD85, Prop. VI.4.1]). It is of real, resp., quaternionic type if and only if an invariant symmetric, resp., skew-symmetric bilinear form exists ([BtD85, Prop. II.6.4]), and this can also be read from the highest weight ([BtD85, Prop. VI.4.6]).

Further, for any automorphism $\sigma \in \operatorname{Aut}\left(G_{1}\right)$, there exists an inner automorphism $\sigma^{\prime}$ such that $\tau:=\sigma \sigma^{\prime}$ preserves $T$. Then $\lambda^{\tau}:=\left.\lambda \circ \tau\right|_{T}$ is an extremal weight of $U_{\lambda} \circ \tau \cong U_{\lambda} \circ \sigma$, 
so that $U_{\lambda}^{*} \circ \tau \cong U_{\lambda}$ if and only if $-\lambda^{\tau} \in \mathcal{W} \lambda$.

The following lemma shows that, if only $G_{1}$ and an involutive automorphism $\tau$ of $G_{1}$ are given, then there always exists an extension to a group of the type $G_{1} \rtimes_{\alpha} \mathbb{Z}_{4}$, where $\alpha_{\overline{1}}=\tau$. This issue is already discussed in Wigner's book Wig59, §26, p. 329], where $J^{2}= \pm \mathbf{1}$ is related to spin being integral or half-integral.

Lemma 2.16. Let $(U, \mathcal{H})$ be a unitary representation of the group $G_{1}$ and $\tau \in \operatorname{Aut}\left(G_{1}\right)$ be an involution. If $U \circ \tau \cong U^{*}$, then there exists a $J \in \mathrm{AU}(\mathcal{H})$ with $J^{4}=\mathbf{1}$ and $J U_{g} J^{-1}=U_{\tau(g)}$ for $g \in G_{1}$.

Proof. From $U \circ \tau \cong U^{*}$ we obtain a $J \in \mathrm{AU}(\mathcal{H})$ with $J U_{g} J^{-1}=U_{\tau(g)}$ for $g \in G_{1}$. As $\tau^{2}=\operatorname{id}_{G_{1}}$, the unitary operator $J^{2}$ commutes with $U_{G_{1}}$. We therefore have a $G_{1}$-invariant orthogonal decomposition $\mathcal{H}=\mathcal{H}_{+} \oplus \mathcal{H}_{-}$, where $\mathcal{H}_{-}=\operatorname{ker}\left(J^{2}+1\right)$ and $\mathcal{H}_{+}=\mathcal{H}_{-}^{\perp}$. Since both subspaces are invariant under $G_{1}$ and $J$, we may w.l.o.g. assume that $\mathcal{H}_{-}=\{0\}$ and show that there exists a conjugation commuting with $G_{1}$.

Conjugating with $J$ defines an antilinear automorphism of the von Neumann algebra $\mathcal{M}:=U_{G_{1}}^{\prime}$ fixing the unitary element $J^{2}$. Therefore Lemma A.1 implies the existence of a unitary $A \in U_{G_{1}}^{\prime}$ with $J A J=A$ and $A^{2}=J^{2}$. Replacing $J$ by $\widetilde{J}:=A^{-1} J$, we obtain $\widetilde{J}^{2}=1$.

Lemma 2.17. Let $G_{1}$ be an abelian group, $\tau(g)=g^{-1}$ and $G:=G_{1} \rtimes\{\mathbf{1}, \tau\}$. Then every unitary representation of $G_{1}$ extends to an antiunitary representation of $G$.

Proof. We consider $G_{1}$ as a discrete group, so that any unitary representation $(U, \mathcal{H})$ of $G_{1}$ is a direct sum of cyclic representations of the form $\left(V, L^{2}\left(\widehat{G_{1}}, \mu\right)\right)$, where $\left(V_{g} f\right)(\chi)=$ $\chi(g) f(\chi)$. Then $J f:=\bar{f}$ defines a conjugation on $L^{2}(\widehat{A}, \mu)$ with $J V_{g} J=V_{g^{-1}}$, so that we obtain an extension of $V$ to an antiunitary representation of $G$.

LEMma 2.18. Suppose that $G \cong G_{1} \rtimes\{\mathrm{id}, \tau\}$, where $\tau \in \operatorname{Aut}(G)$ is an involution.

(i) If $(U, \mathcal{H})$ is an irreducible antiunitary representations of $G$ and $x \in \mathfrak{g}^{\tau}$ satisfies $-i \mathrm{~d} U(x) \geq 0$, then $\mathrm{d} U(x)=0$.

(ii) If $(U, \mathcal{H})$ is an irreducible unitary representations of $G_{1}$ and $x \in \mathfrak{g}^{\tau}$ satisfies $-i \mathrm{~d} U(x) \geq 0$ and $\mathrm{d} U(x) \neq 0$, then $U^{*} \circ \tau \neq U$, i.e., $U$ is of complex type with respect to $\tau$.

Proof. (i) The conjugation $U_{\tau}$ on $\mathcal{H}$ satisfies $U_{\tau} i \mathrm{~d} U(x) U_{\tau}=-i \mathrm{~d} U(\tau x)=-i \mathrm{~d} U(x)$, so that the positivity assumption implies $\mathrm{d} U(x)=0$.

(ii) From (i) it follows that $U$ does not extend to an antiunitary representation of $G$. By Theorem 2.11(d)(ii), $V:=U \oplus U^{*} \circ \tau$ extends to an irreducible antiunitary representation of $G$. If $V_{G}^{\prime} \cong \mathbb{H}$, then $U^{*} \circ \tau \cong U$ implies $-i \mathrm{~d} V(x) \geq 0$, so that $\mathrm{d} V(x)=0$ by (i), and this contradicts $\mathrm{d} U(x) \neq 0$. We conclude that $V_{G}^{\prime} \cong \mathbb{C}$ and $U^{*} \circ \tau \neq U$.

REMARK 2.19. In OM16] the authors study a concept of a "Wigner elementary relativistic system" which is defined as a faithful irreducible orthogonal representation $(U, \mathcal{K})$ of the proper orthochronous Poincaré group $G:=P(4)_{+}^{\uparrow}$ on a real Hilbert space $\mathcal{K}$. Writing $\left(\widetilde{P}_{j}\right)_{0 \leq j \leq 3}$ for the skew-adjoint generators of the unitary representation of the translations 
groups $U_{t e_{j}}=e^{t \widetilde{P}_{j}}$, the mass squared operator is defined as

$$
M^{2}:=-\widetilde{P}_{0}^{2}+\sum_{j=1}^{3} \widetilde{P}_{j}^{2} .
$$

One of the main results in OM16] is that if $M^{2} \geq 0$, then $\mathcal{K}$ carries a complex structure $I$ commuting with the image of $U$ ([0M16, Thm. 4.3, Thm. 5.11]).

This result can be obtained quite directly in our context. We consider the complexification $\left(U_{\mathbb{C}}, \mathcal{K}_{\mathbb{C}}\right)$ of the representation on $\mathcal{K}$ by extending all operators $U_{g}$ to unitary operators on $\mathcal{K}_{\mathbb{C}}$. Then the operators $P_{j}:=-i \widetilde{P}_{j}$ are selfadjoint with

$$
M^{2}=P_{0}^{2}-\sum_{j=1}^{3} P_{j}^{2} \geq 0 .
$$

Since $(U, \mathcal{K})$ is irreducible, its commutant is isomorphic to $\mathbb{R}, \mathbb{C}$ or $\mathbb{H}$ ( $[$ StVa02, Thm. 1]). We claim that it is isomorphic to $\mathbb{C}$. If this is not the case, then $U_{\mathbb{C}}$ is either irreducible (if the commutant is $\mathbb{R}$ ) or a direct sum of two copies of the same irreducible unitary representation $(\widehat{U}, \widehat{\mathcal{H}})$ of $G$ (if the commutant is $\mathbb{H}$ ). As $M^{2} \geq 0$, the spectrum of the translation group is contained in the set

$$
D:=\left\{\left(x_{0}, \mathbf{x}\right) \in \mathbb{R}^{1,3}: x_{0}^{2} \geq \mathbf{x}^{2}\right\} .
$$

The decomposition $D=D_{+} \dot{\cup}\{0\} \cup \dot{U} D_{-}$with $D_{ \pm}:=\left\{x \in D: \pm x_{0}>0\right\}$ is invariant under $\mathrm{SO}_{1,3}(\mathbb{R})^{\uparrow}$, so that we obtain a corresponding decomposition $\widehat{U}=\widehat{U}^{+} \oplus \widehat{U}^{0} \oplus \widehat{U}^{-}$, where the spectrum of $\left.\widehat{U}_{j}\right|_{\mathbb{R}^{1,3}}$ is supported by $D_{j}$. Since $\widehat{U}$ is irreducible, only one summand is non-zero. Further, $\widehat{U}=\widehat{U}_{0}$ implies that the translation group acts trivially, which is ruled out by the assumption that $U$ is faithful. Hence we may w.l.o.g. assume that $\widehat{U}=\widehat{U}_{+}$, so that $P_{0}>0$ (i.e., $P_{0} \geq 0$ and ker $\left.P_{0}=\{0\}\right)$ on $\widehat{\mathcal{H}}$ and therefore on $\mathcal{H}_{\mathbb{C}}$. Next we observe that the conjugation $J$ of $\mathcal{H}_{\mathbb{C}}$ with respect to $\mathcal{H}$ commutes with $\widetilde{P}_{0}$, hence satisfies $J P_{0} J=-P_{0}$, which leads to the contradiction $P_{0}=0$ because it implies that the spectrum of $P_{0}$ is symmetric (cf. Remark 2.24 below). This shows that the commutant $U_{G}^{\prime}$ is $\mathbb{C}$, so that there exists an, up to sign unique, complex structure on $\mathcal{H}$ commuting with $U_{G}$.

2.3. One-parameter groups. We have seen in Example 2.8 that there are three types of one-dimensional Lie groups defining involutive group pairs:
(A) $\mathbb{R}^{\times}$, resp., $\left(\mathbb{R}^{\times}, \mathbb{R}_{+}^{\times}\right)$,
(B) $\mathbb{R} \rtimes\{ \pm$ id $\}$, and
(C) $\operatorname{Pin}_{2}(\mathbb{R})$.

Before we turn to the most important case $(\mathrm{A})$, we take a brief look at the other two cases.

REMARK 2.20. Case (B): Here any antiunitary representation $(U, \mathcal{H})$ yields a conjugation $J:=U_{(0,-1)}$ which defines a real structure on $\mathcal{H}$ and satisfies $J U_{t} J=U_{-t}$ for $t \in \mathbb{R}$. Conversely, every unitary one-parameter group extends to an antiunitary representation of $G$ (Lemma 2.17). 
Case (C): For the group $G=\mathbb{T}\{\mathbf{1}, J\}=\operatorname{Pin}_{2}(\mathbb{R})$, we have $J^{2}=-\mathbf{1}$ and $J z J^{-1}=\bar{z}$ for $z \in \mathbb{T}$, so that antiunitary representations correspond to pairs $(H, I)$, where $I \in \mathrm{AU}(\mathcal{H})$ satisfies $I^{4}=1$ and $H$ is a selfadjoint operator satisfying $I H I=H$ and $e^{\pi i H}=I^{2}$. This implies in particular that $\operatorname{Spec}(H) \subseteq \mathbb{Z}$. For any such pair we put $U_{J}:=I$ and $U_{e^{i t}}:=e^{i t H}$ (see [NÓ16, §4.5] for a natural occurence of such representations).

The following simple observation is the fundamental link between modular theory and antiunitary representations.

Lemma 2.21. For every continuous antiunitary representation $(U, \mathcal{H})$ of $\mathbb{R}^{\times}$and the infinitesimal generator $H$ defined by $U_{e^{t}}=e^{i t H}$, we obtain by

$$
\Delta:=e^{H} \quad \text { and } \quad J:=U_{-1}
$$

a pair $(\Delta, J)$, consisting of a positive operator $\Delta$ and a conjugation $J$ satisfying the modular relation

$$
J \Delta J=\Delta^{-1} .
$$

Conversely, any such pair $(\Delta, J)$ defines an antiunitary representation of $\mathbb{R}^{\times}$by

$$
U_{e^{t}}:=\Delta^{-i t / 2 \pi} \quad \text { and } \quad U_{-1}:=J .
$$

Proof. The only point one has to observe here is that the antiunitarity of $J$ implies that $J U_{t} J=U_{t}$ corresponds to the relation $J H J=-H$, which is equivalent to $J \Delta J=\Delta^{-1}$.

Lemma 2.21 motivates the following definition from the perspective of antiunitary representations:

Definition 2.22. A pair of modular objects on a complex Hilbert space $\mathcal{H}$ is a pair $(\Delta, J)$, where $J$ is a conjugation, i.e., an antilinear isometric involution and $\Delta>0$ is a positive selfadjoint operator satisfying the modular relation (3). Then $J$ is called the modular conjugation and $\Delta$ the modular operator.

With this terminology, the preceding lemma immediately yields:

Corollary 2.23. For any continuous homomorphism $\gamma:\left(\mathbb{R}^{\times}, \mathbb{R}_{+}^{\times}\right) \rightarrow\left(G, G_{1}\right)$ and any continuous antiunitary representation $(U, \mathcal{H})$ of $\left(G, G_{1}\right)$, we obtain a pair of modular objects $\left(\Delta_{\gamma}, J_{\gamma}\right)$ from the representation $U \circ \gamma$ of $\mathbb{R}^{\times}$.

REMARK 2.24. For a selfadjoint operator $H$, the existence of a conjugation $J$ satisfying $J H J=-H$ is equivalent to the restriction $H(0, \infty)$ to the strictly positive spectral subspace being equivalent to the restriction $H(-\infty, 0)$ to the strictly negative spectral subspace ([Lo08] ). Only such operators $H$ arise as infinitesimal generators for antiunitary representations of $\mathbb{R}^{\times}$.

EXAmple 2.25. Let $\left(G, G_{1}\right)$ be an involutive pair of Lie groups and $r \in G \backslash G_{1}$ be such that $\tau:=\left.c_{r}\right|_{G_{1}}$ is an involution. Then $\operatorname{Ad}(\tau)$ is an involutive automorphism of $\mathfrak{g}$ and if $\mathfrak{g}$ is non-abelian, then $\mathfrak{g}^{\tau} \neq\{0\}$.

(A) If $r^{2}=\mathbf{1}$, then any element $x \in \mathfrak{g}^{\tau}$ leads to a homomorphism

$$
\gamma_{r, x}: \mathbb{R}^{\times} \rightarrow G, \quad \gamma_{r, x}\left(e^{t}\right):=\exp (t x), \quad \gamma_{r, x}(-1):=r .
$$


(B) If $r^{2}=\mathbf{1}$, then any element $x \in \mathfrak{g}^{-\tau}$ leads to a homomorphism

$$
\gamma_{r, x}: \mathbb{R} \rtimes\left\{ \pm \operatorname{id}_{\mathbb{R}}\right\} \rightarrow G, \quad \gamma_{r, x}(t):=\exp (t x), \quad \gamma_{r, x}(-1):=r .
$$

(C) If $r^{4}=\mathbf{1}$, then any element $x \in \mathfrak{g}^{-\tau}$ with $\exp (\pi x)=r^{2}$ leads to a homomorphism

$$
\gamma_{r, x}: \operatorname{Pin}_{2}(\mathbb{R})=\mathbb{T}\{\mathbf{1}, J\} \rightarrow G, \quad \gamma_{r, x}\left(e^{i t}\right):=\exp (t x), \quad \gamma_{r, x}(J):=r .
$$

Definition 2.26. (One-parameter groups of complex type) Let $\left(G, G_{1}\right)$ be an involutive Lie group pair. We assume that $G$ is a subgroup of a complex Lie group $G_{\mathbb{C}}$ on which there exists an antiholomorphic involution $\sigma$ such that $G \subseteq\left(G_{\mathbb{C}}\right)^{\sigma}$. We consider the set

$$
\mathcal{Y}_{\left(G, G_{1}\right)}:=\left\{x \in \mathfrak{g}: 2 \pi=\min \{t>0: \exp (t i x)=e\}, \exp (\pi i x) \in G \backslash G_{1}\right\} .
$$

We associate to each $x \in \mathcal{Y}_{\left(G, G_{1}\right)}$ the holomorphic homomorphism

$$
\gamma_{x}: \mathbb{C}^{\times} \rightarrow G_{\mathbb{C}}, \quad \gamma_{x}\left(e^{z}\right):=\exp (z x) .
$$

Then $\sigma\left(\gamma_{x}(w)\right)=\gamma_{x}(\bar{w})$ for $w \in \mathbb{C}^{\times}$and thus $\gamma_{x}\left(\mathbb{R}^{\times}\right) \subseteq\left(G_{\mathbb{C}}\right)^{\sigma}$ holds automatically and $r_{x}:=\gamma_{x}(-1)$ is an involution. For $x \in \mathcal{Y}_{\left(G, G_{1}\right)}$, we thus obtain

$$
\gamma_{x} \in \operatorname{Hom}\left(\left(\mathbb{R}^{\times}, \mathbb{R}_{+}^{\times}\right),\left(G, G_{1}\right)\right) .
$$

In Section 5 below we shall see that many geometric realizations of modular automorphism groups come from elements of $\mathcal{Y}_{\left(G, G_{1}\right)}$, where $G=P(d)_{+}$is the Poincaré group or the conformal group $\operatorname{Conf}\left(\mathbb{R}^{1, d-1}\right) \cong \mathrm{O}_{2, d}(\mathbb{R}) /\{ \pm \mathbf{1}\}$ of Minkowski space (cf. HN12, $\S 17.4])$. This motivates the following discussion of examples.

ExAmple 2.27. (a) For $\left(G, G_{1}\right)=\left(\mathbb{R}^{\times}, \mathbb{R}_{+}^{\times}\right)$and $G_{\mathbb{C}}=\mathbb{C}^{\times}$and $\exp (z)=e^{z}$, we have $\mathcal{Y}_{\left(G, G_{1}\right)}=\{ \pm 1\} \subseteq \mathbb{R}=\mathfrak{g}$.

(b) (Lorentz groups) For

$$
G=\mathrm{SO}_{1,1}(\mathbb{R}) \subseteq G_{\mathbb{C}}=\mathrm{SO}_{1,1}(\mathbb{C})=\left\{\left(\begin{array}{ll}
a & b \\
b & a
\end{array}\right): a, b \in \mathbb{C}, a^{2}-b^{2}=1\right\},
$$

we have $G \cong \mathbb{R}^{\times}$and $G_{\mathbb{C}} \cong \mathbb{C}^{\times}$, so that we basically have the same situation as under (a). Here a canonical generator of the Lie algebra is the boost generator

$$
b_{0}:=\left(\begin{array}{ll}
0 & 1 \\
1 & 0
\end{array}\right) \quad \text { with } \quad e^{z b_{0}}=\left(\begin{array}{cc}
\cosh z & \sinh z \\
\sinh z & \cosh z
\end{array}\right) \quad \text { and } \quad r_{b_{0}}=e^{\pi i b_{0}}=\mathbf{- 1} .
$$

We have $\mathcal{Y}_{\left(G, G_{0}\right)}=\left\{ \pm b_{0}\right\}$.

This example embeds naturally into the higher dimensional Lorentz groups $G=$ $\mathrm{SO}_{1, d}(\mathbb{R}) \subseteq G_{\mathbb{C}}=\mathrm{SO}_{1, d}(\mathbb{C})$, where

$$
b_{0}:=E_{10}+E_{01} \in \mathcal{Y}_{\left(G, G_{0}\right)} \quad \text { and } \quad r_{b_{0}}=R_{01}=\operatorname{diag}(-1,-1,1, \ldots, 1) .
$$

Since the simple real Lie algebra $\mathfrak{g}=\mathfrak{s o}_{1, d}(\mathbb{R})$ (for $d \geq 2$ ) is of real rank 1 , all addiagonalizable elements $x \in \mathfrak{g}$ are conjugate to a multiple of $b_{0}$. All these elements $x$ are diagonalizable matrices and $\operatorname{im}(x)$ is a two-dimensional Minkowski plane in which the two eigenvectors are light-like. Conversely, every triple $\left(\beta, \ell_{+}, \ell_{-}\right)$consisting of $\beta \in \mathbb{R}^{\times}$and two linearly independent light-like vectors $\ell_{ \pm}$specifies such an element $x=x\left(\ell_{+}, \ell_{-}, \beta\right) \in$ $\mathfrak{g}$ by $x \ell_{ \pm}= \pm \beta \ell_{ \pm}$and $\operatorname{ker} x=\left\{\ell_{1}, \ell_{2}\right\}^{\perp}$. We then have

$$
\mathcal{Y}_{\left(G, G_{0}\right)}=\operatorname{Ad}(G) b_{0}=\left\{x\left(\ell_{+}, \ell_{-}, \beta\right): \beta=1\right\} \cong \mathrm{SO}_{1, d}(\mathbb{R}) /\left(\mathrm{SO}_{1,1}(\mathbb{R}) \times \mathrm{SO}_{d-1}(\mathbb{R})\right),
$$


and this is a symmetric space because the centralizers of $b_{0}$ and the involution $r_{b_{0}}$ share the same identity component.

(c) For the affine group $G:=\operatorname{Aff}(\mathbb{R}) \cong \mathbb{R} \rtimes \mathbb{R}^{\times}$of the real line, the coset $G \backslash G_{0}$ consists of the orientation reversing affine maps. Note that $G_{\mathbb{C}} \cong \mathbb{C} \rtimes \mathbb{C}^{\times}$and $G_{\mathbb{C}}^{\sigma}=G$. Here $\mathcal{Y}_{\left(G, G_{0}\right)} \cong \mathbb{R} \times\{ \pm 1\}$ is the set of real affine vector fields $X$ for which the vector field $i X$ on $\mathbb{C}$ generates a $2 \pi$-periodic flow (whose center lies on the real axis).

EXAMPLE 2.28. We consider the real projective group $G=\mathrm{PGL}_{2}(\mathbb{R}) \subseteq G_{\mathbb{C}}=\mathrm{PGL}_{2}(\mathbb{C})$ acting on the real projective line $\mathbb{S}^{1} \cong \mathbb{R} \cup\{\infty\}$, resp., on the Riemann sphere $\mathbb{C} \cup\{\infty\} \cong$ $\mathbb{P}_{1}(\mathbb{C})$.

A subset $I \subset \mathbb{S}^{1}$ is called an interval if it is connected, open, non-empty and not dense. Then the interior $I^{\prime}$ of its complement also is an interval. For every interval there is a canonical involution $r_{I} \in \mathrm{PGL}_{2}(\mathbb{R})$ fixing both endpoints and exchanging $I$ and $I^{\prime}$. The centralizer of $r_{I}$ in $\mathrm{PSL}_{2}(\mathbb{R})$ is isomorphic to $\mathrm{PSO}_{1,1}(\mathbb{R}) \cong \mathbb{R}$, hence connected, and there exists an element $x_{I} \in \mathcal{Y}_{\left(G, G_{0}\right)}$ which is up to sign unique. The corresponding homomorphism $\gamma^{I}:=\gamma_{x_{I}}: \mathbb{R}^{\times} \rightarrow G$ satisfies $\gamma_{-1}^{I}=\exp \left(\pi i x_{I}\right)=r_{I}$.

For the interval $I=(0, \infty)$, we have

$$
\gamma_{t}^{I}(z)=t z \quad \text { and } \quad r_{I}(z)=-z .
$$

For $I=(-1,1)$, we have $\gamma^{I}\left(\mathbb{R}^{\times}\right)=\mathrm{PO}_{1,1}(\mathbb{R})$ and $\gamma_{2 t}^{I}(z):=\frac{\cosh t \cdot z+\sinh t}{\sinh t \cdot z+\cosh t}$. This leads to

$$
\gamma_{2 t i}^{I}(z)=\frac{\cos t \cdot z+i \sin t}{i \sin t \cdot z+\cos t}, \quad \text { so that } \quad \gamma_{2 \pi i}^{I}(z)=z \quad \text { and } \quad r_{I}(z)=\gamma_{\pi i}^{I}(z)=\frac{1}{z} .
$$

\subsection{Some low-dimensional groups.}

2.4.1. The affine group of the real line. We consider the affine group $G:=\operatorname{Aff}(\mathbb{R})=$ $\mathbb{R} \rtimes \mathbb{R}^{\times}$and its identity component $G_{1}=\mathbb{R} \rtimes \mathbb{R}_{+}^{\times}$. We say that a unitary representation $(U, \mathcal{H})$ of $G_{1}$ is of positive energy if $U_{(t, 1)}=e^{i t P}$ with $P \geq 0$, i.e., the restriction to the translation subgroup has non-negative spectrum. We speak of strictly positive energy if, in addition, $\operatorname{ker} P=\{0\}$.

Up to unitary equivalence, $G_{1}$ has exactly one irreducible unitary representation with strictly positive energy and every unitary representation with strictly positive energy is a multiple of the irreducible one. The analogous statement holds for negative energy ([Lo08, Thm. 2.8]). Further, any unitary representation $U$ of $G_{1}$ decomposes uniquely as a direct sum $U=U^{+} \oplus U^{0} \oplus U^{-}$, where $U^{ \pm}$have strictly positive/negative energy and the translation group is contained in $\operatorname{ker} U^{0}$.

The unique irreducible representation of strictly positive energy can be realized on $\mathcal{H}:=L^{2}\left(\mathbb{R}^{+}\right)$by

$$
\left(U_{\left(t, e^{s}\right)} f\right)(x)=e^{i t x} e^{s / 2} f\left(e^{s} x\right) .
$$

It obviously extends by $U_{(0,-1)} f:=\bar{f}$ to an irreducible antiunitary representation of $G$. By Theorem 2.11 we thus obtain up to equivalence precisely one irreducible antiunitary representation of $G$ with strictly positive energy. More generally, we have by Lo08, Prop. 2.11] and Theorem 2.11,

Proposition 2.29. Every unitary representation $(U, \mathcal{H})$ of $\operatorname{Aff}(\mathbb{R})_{0}$ of strictly positive energy extends to an antiunitary representation $\bar{U}$ of $\mathrm{Aff}(\mathbb{R})$ on the same Hilbert space 
which is unique up to equivalence.

The representation theory of the affine group can be used to draw some general conclusions on spectra of one-parameter groups.

Proposition 2.30. Let $G$ be a connected Lie group and $(U, \mathcal{H})$ be a unitary representation for which $\mathrm{d} U$ is faithful and $x \in \mathfrak{g}$. Then the following assertions hold:

(a) If $\mathrm{ad} x$ has a non-zero real eigenvalue, then $\operatorname{Spec}(i \mathrm{~d} U(x))=\mathbb{R}$.

(b) If $\mathfrak{g}$ is semisimple and $0 \neq y$ is nilpotent, then $\operatorname{Spec}(i \mathrm{~d} U(y)) \in\left\{\mathbb{R}, \mathbb{R}_{+}, \mathbb{R}_{-}\right\}$.

(c) If $0 \neq x \in \mathfrak{g}$ is such that $\operatorname{ad} x$ is diagonalizable and $\mathfrak{b} \unlhd \mathfrak{g}$ is the ideal generated by $\operatorname{im}(\operatorname{ad} x)$ and $x$, then $\operatorname{ker}(\mathrm{d} U(x))=\mathcal{H}^{B}=\left\{\xi \in \mathcal{H}:(\forall g \in B) U_{g} \xi=\xi\right\}$ holds for the corresponding integral subgroup $B \unlhd G$.

Proof. (a) Let $0 \neq y \in \mathfrak{g}$ with $[x, y]=\lambda y$ for some $\lambda \neq 0$. Then $\mathfrak{h}:=\mathbb{R} x+\mathbb{R} y$ is a 2-dimensional non-abelian subalgebra and $\mathrm{d} U(y) \neq 0$. Therefore the assertion follows from the fact that, for all irreducible unitary representations of the corresponding 2dimensional subgroup isomorphic to $\operatorname{Aff}(\mathbb{R})_{0}$, the spectrum of $i \mathrm{~d} U(x)$ coincides with $\mathbb{R}$.

(b) Using the Jacobson-Morozov Theorem, we find an $h \in \mathfrak{g}$ with $[h, x]=x$, so that the Lie algebra $\mathfrak{b}:=\mathbb{R} h+\mathbb{R} x$ is isomorphic to $\mathfrak{a f f}(\mathbb{R})$. Then the result follows from the classification of the irreducible representations of the group $\exp (\mathfrak{b}) \cong \operatorname{Aff}(\mathbb{R})$.

(c) Let $\mathfrak{g}=\oplus_{\mu} \in \mathbb{R} \mathfrak{g}_{\mu}(\operatorname{ad} x)$ denote the eigenspace decomposition of $\mathfrak{g}$ with respect to the diagonalizable operator ad $x$. Then the representation theory of $\operatorname{Aff}(\mathbb{R})_{0}$ implies that, for $\mu \neq 0$, the operators in $\mathrm{d} U\left(\mathfrak{g}_{\mu}(\operatorname{ad} x)\right)$ vanish on ker $\mathrm{d} U(x)$. This shows that the Lie subalgebra $\mathfrak{h}$ generated by $x$ and $[x, \mathfrak{g}]$ acts trivially on ker $\mathrm{d} U(x)$. Since this subalgebra is invariant under $\operatorname{ad}\left(\mathfrak{g}_{0}(\operatorname{ad} x)\right)$ and contains the other eigenspaces of ad $x$, it is an ideal of $\mathfrak{g}$, hence coincides with $\mathfrak{b}$. Therefore $\operatorname{ker}(\mathrm{d} U(x))=\mathcal{H}^{B}$.

2.4.2. The projective group of the real line. We consider the projective group $G=$ $\mathrm{PGL}_{2}(\mathbb{R})$ and its identity component $G_{1}=\mathrm{PSL}_{2}(\mathbb{R})$. We write $r(x)=-x$ for the reflection in 0 which commutes with the dilation group $\mathbb{R}^{\times} \subseteq \operatorname{Aff}(\mathbb{R}) \subseteq \mathrm{PGL}_{2}(\mathbb{R})$ (cf. Example 2.28). Note that $r$ extends to an antiholomorphic automorphism $r(z):=-\bar{z}$ of the upper half plane $\mathbb{C}_{+}$, so that we obtain an identification of $G$ with the group AAut $\left(\mathbb{C}_{+}\right)$ (Example 2.9(d)).

For the generators of $\mathfrak{s l}_{2}(\mathbb{R})$, we write

$$
T=\left(\begin{array}{ll}
0 & 1 \\
0 & 0
\end{array}\right), \quad S=\left(\begin{array}{cc}
0 & 0 \\
-1 & 0
\end{array}\right) \quad \text { and } \quad E=\frac{1}{2}\left(\begin{array}{cc}
1 & 0 \\
0 & -1
\end{array}\right) .
$$

They satisfy the commutation relations

$$
[E, T]=T, \quad[E, S]=-S \quad \text { and } \quad[T, S]=-2 E .
$$

In the complexification $\mathfrak{s l}_{2}(\mathbb{C})$, we have the basis

$$
L_{ \pm 1}:=\frac{1}{2}\left(\begin{array}{cc}
1 & \mp i \\
\mp i & -1
\end{array}\right)=E \mp \frac{i}{2}(T-S), \quad L_{0}:=-\frac{i}{2}\left(\begin{array}{cc}
0 & 1 \\
-1 & 0
\end{array}\right)=-\frac{i}{2}(T+S) .
$$

These elements satisfy the relations

$$
\left[L_{0}, L_{-1}\right]=L_{-1}, \quad\left[L_{0}, L_{1}\right]=-L_{1} \quad \text { and } \quad\left[L_{1}, L_{-1}\right]=-2 L_{0} .
$$


Definition 2.31. The element $L_{0} \in i \mathfrak{s l}_{2}(\mathbb{R})$ is called the conformal Hamiltonian. A unitary representation $(U, \mathcal{H})$ of $\widetilde{\mathrm{SL}}_{2}(\mathbb{R})$ is called a positive energy representation if $\mathrm{d} U\left(L_{0}\right) \geq 0$.

The following result is well known; for a proof in the spirit of the present exposition, we refer to [Lo08, Cor. 2.9].

COROllary 2.32. For every non-trivial irreducible positive energy representation $U$ of the simply connected covering group $\widetilde{\mathrm{SL}_{2}}(\mathbb{R})$, the restriction to $\mathrm{Aff}(\mathbb{R})_{0}$ is also irreducible. If $U$ is non-trivial, then $\left.U\right|_{\mathrm{Aff}(\mathbb{R})_{0}}$ is the unique irreducible representation with strictly positive energy.

REMARK 2.33. In $\operatorname{PSL}_{2}(\mathbb{R})$, we have $\exp \left(2 \pi i L_{0}\right)=\mathbf{1}$, so that, for every irreducible positive energy representation of $\mathrm{PSL}_{2}(\mathbb{R})$, the spectrum of $\mathrm{d} U\left(L_{0}\right)$ is contained in $m+\mathbb{N}_{0}$ for some $m \in \mathbb{N}_{0}$. We call $m$ its lowest weight and write $\mathcal{H}_{m}=\mathbb{C} \xi_{m}$ for the $m$-eigenspace of $L_{0}$ in $\mathcal{H}$. Then $\mathrm{d} U\left(L_{1}\right) \xi_{m}=0$ and $\xi_{m+k}:=\mathrm{d} U\left(L_{-1}\right)^{k} \xi_{m}, k \in \mathbb{N}_{0}$, is an orthogonal basis of $\mathcal{H}$.

Theorem 2.34. ([Lo08, Thm. 2.10]) Every unitary positive energy representation $U$ of $\mathrm{PSL}_{2}(\mathbb{R})$ extends to an antiunitary representation $\bar{U}$ of $\mathrm{PGL}_{2}(\mathbb{R})$ on the same Hilbert space. This extension is unique up to isomorphism and, if $U$ is irreducible, then $J:=\bar{U}_{r}$ (for $r(x)=-x$ ) is unique up to a multiplicative factor in $\mathbb{T}$.

Proof. In view of Theorem 2.11, it suffices to verify the first assertion.

Here the main point is to define the antiunitary involution on the irreducible lowest weight representation $U^{m}$ of lowest weight $m \in \mathbb{N}_{0}$. We specify an antiunitary involution $C$ on $\mathcal{H}$ by $C \xi_{n}=\xi_{n}$ for $n \geq m$ (cf. Remark 2.33). Then $C$ commutes with $L_{0}$ and $L_{ \pm 1}$ and

$$
C E C=E, \quad C T C=-T \quad \text { and } \quad C S C=-S .
$$

This implies that $C U_{g}^{m} C=U_{r g r}^{m}$ for $g \in \mathrm{PSL}_{2}(\mathbb{R})$.

Definition 2.35. (Positive energy representations) (a) A unitary representation $(U, \mathcal{H})$ of the translation group $\mathbb{R}^{d}=\mathbb{R}^{1, d-1}$ of Minkowski space is said to be a positive energy representation if $-i \mathrm{~d} U(x) \geq 0$ for $x \in \overline{V_{+}}$.

(b) A unitary representation $(U, \mathcal{H})$ of the Poincaré group $P(d)_{+}^{\uparrow}$ is said to be a positive energy representation if its restriction to the translation subgroup is of positive energy. We likewise define antiunitary positive energy representations of $P(d)_{+}$.

REMARK 2.36. (a) For the group $G:=P(2)_{+} \cong \mathbb{R}^{1,1} \rtimes \mathrm{SO}_{1,1}(\mathbb{R})$ and the reflection $r:=(0,-\mathbf{1})$ inducing on $G$ the involution $\tau(b, a)=(-b, a)$, there are similar results to Theorem 2.34 (cf. Theorem [3.19). Here the main point is to see that the irreducible strictly positive energy representations $(U, \mathcal{H})$ of $G_{0}$ carry a natural conjugation that we can use for the extension. In the $L^{2}$-realization on the hyperbolas

$$
\mathcal{O}_{m}=\left\{(\lambda, \mu) \in \mathbb{R}^{2}: \lambda^{2}-\mu^{2}=m^{2}\right\}, \quad m>0,
$$

suggested by Mackey theory, we can extend the representation simply by $U_{(0,0,-\mathbf{1})} f=\bar{f}$.

(b) For the Poincaré group $P(d)_{+}$, the situation is more complicated. The irreducible strictly positive energy representations of $P(d)_{+}^{\uparrow} \cong \mathbb{R}^{d} \rtimes \mathrm{SO}_{1, d-1}(\mathbb{R})^{\uparrow}$ are induced from 
representations of the stabilizer group $\mathrm{SO}_{1, d-1}(\mathbb{R})_{e_{0}} \cong \mathrm{SO}_{d-1}(\mathbb{R})$ and realized in vectorvalued $L^{2}$-spaces on the hyperboloids

$$
\mathcal{O}_{m}=\left\{\left(p_{0}, \mathbf{p}\right) \in \mathbb{R}^{d}: p_{0}^{2}-\mathbf{p}^{2}=m^{2}\right\}, \quad m>0 .
$$

Since the stabilizer group is non-trivial for $d>2$, the existence of an antiunitary extension to $P(d)_{+}$depends on the existence of an antiunitary extension of the representation $(\rho, V)$ of $\mathrm{SO}_{d-1}(\mathbb{R})$ to $\mathrm{O}_{d-1}(\mathbb{R})$. We refer to $\mathrm{NO} 17$ for a detailed analysis of these issues; see also [Va85, Thm. 9.10] for a discussion concerning the Poincaré group.

2.4.3. The Heisenberg group. In this subsection we recall the close connection between unitary representations of the 3-dimensional Heisenberg group and positive energy representations of Aff $(\mathbb{R})_{0}$ (cf. [Lo08, Thm. 2.8]) which also extends to antiunitary extensions.

We define the Heisenberg group $\operatorname{Heis}\left(\mathbb{R}^{2}\right)$ as the manifold $\mathbb{T} \times \mathbb{R}^{2}$, endowed with the group multiplication

$$
(z, s, t)\left(z^{\prime}, s^{\prime}, t^{\prime}\right)=\left(z z^{\prime} e^{i s^{\prime} t}, s+s^{\prime}, t+t^{\prime}\right)
$$

Note that

$$
\operatorname{Heis}\left(\mathbb{R}^{2}\right) \cong(\mathbb{T} \times \mathbb{R}) \rtimes_{\alpha} \mathbb{R} \quad \text { for } \quad \alpha_{t}(z, s)=\left(z e^{i s t}, s\right) .
$$

Extending the action of $\mathbb{R}$ on $\mathbb{T} \times \mathbb{R}$ to an action of $\mathbb{R} \times \mathbb{Z}_{2} \cong \mathbb{R}^{\times}$via

$$
\beta_{r}(z, s)=\left(z r^{i s}, s\right) \quad \text { and } \quad \beta_{-1}(z, s)=(\bar{z},-s),
$$

we obtain the larger group

$$
\operatorname{Heis}\left(\mathbb{R}^{2}\right)_{\tau} \cong(\mathbb{T} \times \mathbb{R}) \rtimes_{\beta} \mathbb{R}^{\times} \cong \operatorname{Heis}\left(\mathbb{R}^{2}\right) \rtimes\{\mathbf{1}, \tau\}, \quad \text { with } \quad \tau(z, s, t)=(\bar{z},-s, t) .
$$

Proposition 2.37. There is a natural one-to-one correspondence between unitary representations $(\widetilde{U}, \mathcal{H})$ of Heis $\left(\mathbb{R}^{2}\right)$ satisfying $\widetilde{U}_{(z, 0,0)}=z \mathbf{1}$ and unitary strictly positive energy representations $(U, \mathcal{H})$ of $\operatorname{Aff}(\mathbb{R})_{0}$. It is established as follows:

(i) If $U$ is given and $U_{(b, 1)}=e^{i b P}$ with $P>0$, then we put $W_{s}:=e^{i s \log P}$ and $\widetilde{U}_{(z, s, t)}:=z W_{s} U_{\left(0, e^{t}\right)}$.

(ii) If $\widetilde{U}$ is given and $W_{s}:=\widetilde{U}_{(1, s, 0)}=e^{i s A}$, then we put $U_{\left(s, e^{t}\right)}:=e^{i s \exp A} \widetilde{U}_{(1,0, t)}$.

(iii) This correspondence extends naturally to antiunitary representations of Heis $\left(\mathbb{R}^{2}\right)_{\tau}$ and antiunitary positive energy representations of $\mathrm{Aff}(\mathbb{R})$.

Proof. (i) Let $V_{t}:=U_{\left(0, e^{t}\right)}$ and $A:=\log P$. Then $V_{t} P V_{-t}=e^{t} P$ implies that $W_{s}=e^{i s A}$ satisfies

$$
V_{t} A V_{-t}=t \mathbf{1}+A \quad \text { and } \quad V_{t} W_{s} V_{-t}=e^{i s t} W_{s} .
$$

Therefore $V$ and $W$ define a unitary representation of $\operatorname{Heis}\left(\mathbb{R}^{2}\right)$ via $\widetilde{U}_{(z, s, t)}:=z W_{s} V_{t}$.

(ii) With $V_{t}:=\widetilde{U}_{(1,0, t)}$ and $W_{s}=\widetilde{U}_{(1, s, 0)}=e^{i s A}$, the positive operator $P:=e^{A}$ satisfies $V_{t} P V_{-t}=e^{t} P$, so that we obtain a positive energy representation of $\operatorname{Aff}(\mathbb{R})_{0}$ by $U_{\left(s, e^{t}\right)}:=e^{i s P} V_{t}$.

(iii) If, in addition, $U$ is an antiunitary representation of $\operatorname{Aff}(\mathbb{R}) \cong \mathbb{R} \rtimes \mathbb{R}^{\times}$and $J=U_{(0,-1)}$, then $J U_{(b, a)} J=U_{(-b, a)}$ leads to $J P J=P$ and thus to $J A J=A$. We therefore obtain an antiunitary representation $\widehat{U}$ of $\operatorname{Heis}\left(\mathbb{R}^{2}\right)_{\tau} \cong(\mathbb{T} \times \mathbb{R}) \rtimes_{\alpha} \mathbb{R}^{\times}$by $\widehat{U}_{(z, s, a)}:=\widehat{U}_{(z, s)} U_{(0, a)}$. 
REMARK 2.38. Write Heis $\left(\mathbb{R}^{2}\right)_{\tau}$ as the semidirect product Heis $\left(\mathbb{R}^{2}\right) \rtimes\{\mathbf{1}, \tau\}$, where $\tau(z, s, t)=(\bar{z},-s, t)$. Then the conjugacy class $C_{\tau} \subseteq \operatorname{Heis}\left(\mathbb{R}^{2}\right)_{\tau}$ is a 2-dimensional symmetric space diffeomorphic to $\mathbb{T} \times \mathbb{R}$ and the centralizer of $\tau$ in Heis $\left(\mathbb{R}^{2}\right)$ is the subgroup $\{ \pm 1\} \times\{0\} \times \mathbb{R}$ which also commutes with the whole subgroup $\{(1,0)\} \times \mathbb{R}$. Therefore $C_{\tau}$ can be identified with the conjugacy class of the homomorphism $\gamma: \mathbb{R}^{\times} \rightarrow \operatorname{Heis}\left(\mathbb{R}^{2}\right)_{\tau} \cong$ $(\mathbb{T} \times \mathbb{R}) \rtimes_{\beta} \mathbb{R}^{\times}$with $\gamma(t)=(1,0, t)$.

3. Modular objects and standard subspaces. Besides antiunitary representations of $\mathbb{R}^{\times}$(Lemma 2.21), there are other interesting ways to encode modular objects $(\Delta, J)$. Below we discuss some of them. In particular, we introduce the concept of a standard subspace $V \subseteq \mathcal{H}$ which is a geometric counterpart of antiunitary representations of $\mathbb{R}^{\times}$ (Proposition 3.2). We also discuss how the embedding $V \subseteq \mathcal{H}$ can be obtained from the orthogonal one-parameter group $\left.\Delta^{i t}\right|_{V}$ on $V(\$ 3.3)$, and in 33 we introduce halfsided modular inclusions of standard subspaces and how they are related to antiunitary representations of $\operatorname{Aff}(\mathbb{R}), P(2)+$ and $\mathrm{PGL}_{2}(\mathbb{R})$. Modular intersections are studied in $\$ 3.5$.

3.1. Standard subspaces. We now turn to the fundamental concept of a standard subspace $V$ of a complex Hilbert space $\mathcal{H}$. The key structures on the set $\operatorname{Stand}(\mathcal{H})$ of standard subspaces is a natural action of the group $\operatorname{AU}(\mathcal{H})$, an order structure induced by inclusion, and an involution $V \mapsto V^{\prime}=i V^{\perp_{\mathbb{R}}}$ defined by the symplectic orthogonal space.

Definition 3.1. A closed real subspace $V \subseteq \mathcal{H}$ is called a standard real subspace (or simply a standard subspace) if $V \cap i V=\{0\}$ and $V+i V$ is dense in $\mathcal{H}$. We write $\operatorname{Stand}(\mathcal{H})$ for the set of standard subspaces of $\mathcal{H}$.

For every standard subspace $V \subseteq \mathcal{H}$, we obtain an antilinear unbounded operator

$$
S: \mathcal{D}(S):=V+i V \rightarrow \mathcal{H}, \quad S(v+i w):=v-i w
$$

and this operator is closed, so that $\Delta_{V}:=S^{*} S$ is a positive selfadjoint operator. We thus obtain the polar decomposition

$$
S=J_{V} \Delta_{V}^{1 / 2}
$$

where $J_{V}$ is an antilinear isometry, and $S=S^{-1}=\Delta_{V}^{-1 / 2} J_{V}^{-1}=J_{V}^{-1}\left(J_{V} \Delta_{V}^{-1 / 2} J_{V}^{-1}\right)$ leads to $J_{V}^{-1}=J_{V}$ and the modular relation $J_{V} \Delta_{V} J_{V}=\Delta_{V}^{-1}$. If, conversely, $(\Delta, J)$ is a pair of modular objects, then $S:=J \Delta^{1 / 2}$ is a densely defined antilinear involution and

$$
\operatorname{Fix}(S):=\{\xi \in \mathcal{D}(S): S \xi=\xi\}
$$

is a standard subspace with $J_{V}=J$ and $\Delta_{V}=\Delta$. The correspondence between modular objects and standard subspaces is the core of Tomita-Takesaki Theory (see Theorem 4.2 below).

Combining the preceding discussion with Lemma 2.21, we obtain:

Proposition 3.2. If $(U, \mathcal{H})$ is an antiunitary representation of $\mathbb{R}^{\times}$with $U_{e^{t}}=\Delta^{-i t / 2 \pi}$ for $t \in \mathbb{R}$ and $J:=U_{-1}$, then $V:=\operatorname{Fix}\left(J \Delta^{1 / 2}\right)$ is a standard subspace. This defines a bijection $V \leftrightarrow U^{V}$ between antiunitary representations of $\mathbb{R}^{\times}$and standard subspaces. 
REMARK 3.3. The parametrization of the one-parameter group in Proposition 3.2 may appear artificial, but it turns out that it is quite natural. As $V \subseteq \mathcal{D}\left(\Delta^{1 / 2}\right)$, for each $v \in V$, the orbit map $U^{v}(g):=U_{g} v$ has an analytic extension

$$
\{z \in \mathbb{C}: 0 \leq \operatorname{Im} z \leq \pi\} \rightarrow \mathcal{H}, \quad z \mapsto U_{e^{z}}^{v}:=\Delta^{-i z / 2 \pi} v
$$

with $U^{v}(i \pi)=\Delta^{1 / 2} v=J v$. This fits with $U_{-1}=J$ and it is compatible with the context of Definition 2.26, where $\gamma(-1)=\exp (\pi i x)$ is obtained by analytic continuation from $\gamma\left(e^{t}\right)=\exp (t x)$.

REMARK 3.4. (a) If $V=\operatorname{Fix}(S)$ is a standard subspace with modular objects $(\Delta, J)$, then

$$
\Delta^{1 / 4} S \Delta^{-1 / 4}=\Delta^{1 / 4} J \Delta^{1 / 4}=\Delta^{1 / 4} \Delta^{-1 / 4} J=J
$$

implies that $V=\operatorname{Fix}(S)=\Delta^{-1 / 4} \operatorname{Fix}(J)=\Delta^{-1 / 4} \mathcal{H}^{J}$.

(b) Write $\operatorname{Stand}_{0}(\mathcal{H})$ for the set of those standard subspaces $V$ for which $V+i V=\mathcal{H}$, i.e., the antilinear involution $S$ is bounded. Combining (7) with the fact that the unitary group $\mathrm{U}(\mathcal{H})$ acts transitively on the set of all conjugations (=antiunitary involutions), it follows that the group $\operatorname{GL}(\mathcal{H})$ acts transitively on $\operatorname{Stand}_{0}(\mathcal{H})$. This leads to the structure of a Banach symmetric space on this set

$$
\operatorname{Stand}_{0}(\mathcal{H}) \cong \operatorname{GL}(\mathcal{H}) / \operatorname{GL}\left(\mathcal{H}^{J}\right) \cong \operatorname{GL}(\mathcal{H}) / \mathrm{GL}(\mathcal{H})^{J},
$$

where $J$ is any conjugation on $\mathcal{H}$ (cf. Appendix A.3 and Kl11]). For $\mathcal{H}=\mathbb{C}^{n}$, we obtain in particular

$$
\operatorname{Stand}\left(\mathbb{C}^{n}\right)=\operatorname{Stand}_{0}\left(\mathbb{C}^{n}\right) \cong \mathrm{GL}_{n}(\mathbb{C}) / \mathrm{GL}_{n}(\mathbb{R}) .
$$

For elements of $\operatorname{Stand}_{0}(\mathcal{H})$, there are no proper inclusions. As we shall see in $\$ 3.4$, the order structure on $\operatorname{Stand}(\mathcal{H})$ is non-trivial if $\mathcal{H}$ is infinite dimensional.

(c) To extend (b) to arbitrary standard subspaces $V$, we note that a dense complex subspace $\mathcal{D} \subseteq \mathcal{H}$ carries at most one Hilbert space structure (up to topological linear isomorphism) for which the inclusion $\mathcal{D} \hookrightarrow \mathcal{H}$ is continuous (Closed Graph Theorem). We consider the category $\mathcal{G}$ whose objects are all dense subspaces $\mathcal{D} \subseteq \mathcal{H}$ carrying such Hilbert space structures and whose morphisms are the topological linear isomorphisms $\mathcal{D}_{1} \rightarrow \mathcal{D}_{2}$ with respect to the intrinsic Hilbert space structures. This defines a category in which all morphisms are invertible, so that we actually obtain a groupoid. As all these subspaces $\mathcal{D}$ are isomorphic to $\mathcal{H}$ as Hilbert spaces, this groupoid acts transitively.

For each standard subspace $V \subseteq \mathcal{H}$, the dense subspace $V+i V$ carries the natural Hilbert structure obtained from the identification with the complex Hilbert space $V_{\mathbb{C}}$. Therefore the groupoid $\mathcal{G}$ acts transitively on $\operatorname{Stand}(\mathcal{H})$ with stabilizer groups $\mathcal{G}_{V} \cong$ $\mathrm{GL}(V)$.

(d) Write $\operatorname{Conj}(\mathcal{H})$ for the set of conjugations on $\mathcal{H}$ (Examples 2.4). Then the map $\operatorname{Stand}(\mathcal{H}) \rightarrow \operatorname{AU}(\mathcal{H}), V \mapsto J_{V}$ is surjective and $\operatorname{AU}(\mathcal{H})$-equivariant. The fiber in a fixed conjugation $J$ corresponds to the set of all positive operators $\Delta$ satisfying $J \Delta J=\Delta^{-1}$. Passing to $D:=\left.i \log \Delta\right|_{\mathcal{H}^{J}}$, it follows that it can be parametrized by the set of all skewadjoint operators on the real Hilbert space $\mathcal{H}^{J}$ (see also Remark 3.5(b) for a different parametrization). 
The problem to describe the set of pairs $(V, \mathcal{H})$, where $V \subseteq \mathcal{H}$ is a standard subspace, can be addressed from two directions. One could either start with a real Hilbert space $V$ and ask for all those complex Hilbert spaces into which $V$ embeds as a standard subspace, or start with the pair $(\mathcal{H}, J)$, respectively the real Hilbert space $\mathcal{H}^{J}$, and ask for all standard real subspaces $V \subseteq \mathcal{H}$ with $J_{V}=J$. Both problems have rather explicit answers that are easily explained (see NÓ16] for details).

REMARK 3.5. (a) Let $(V,(\cdot, \cdot))$ be a real Hilbert space. For any realization of $V$ as a standard subspace of $\mathcal{H}$, the restriction of the scalar product of $\mathcal{H}$ to $V$ is a complexvalued hermitian form

$$
h(v, w):=\langle v, w\rangle=(v, w)+i \omega(v, w),
$$

where $\omega: V \times V \rightarrow \mathbb{R}$ is continuous and skew-symmetric, hence of the form $\omega(v, w)=$ $(v, C w)$ for a skew-symmetric operator $C=-C^{\top}$ on $V$ satisfying $\|C v\|<\|v\|$ for any non-zero $v \in V$ (NÓ16, Lemma A.10]). Conversely, we obtain for every such operator $C$ on $V$ by completion of $V_{\mathbb{C}}$ with respect to $h$ a complex Hilbert space in which $V$ is a standard real subspace. Then $C$ extends to a bounded skew-hermitian operator $\widehat{C}$ on $\mathcal{H}$ satisfying

$$
\Delta=\frac{\mathbf{1}-i \widehat{C}}{\mathbf{1}+i \widehat{C}} \quad \text { and } \quad \widehat{C}=i \frac{\Delta-\mathbf{1}}{\Delta+\mathbf{1}} .
$$

(b) If we start with the conjugation $J$ on $\mathcal{H}$, then the standard subspaces $V$ with $J_{V}=J$ are the subspaces of the form $V=(\mathbf{1}+i C) \mathcal{H}^{J}$, where $C \in B\left(\mathcal{H}^{J}\right)$ is a skewsymmetric operator satisfying $\|C v\|<\|v\|$ for $0 \neq v \in \mathcal{H}^{J}$ (Ó16, Lemma B.2]). Writing also $C$ for its complex linear extension to $\mathcal{H}$, we then have

$$
\Delta^{1 / 2}=\frac{\mathbf{1}-i C}{\mathbf{1}+i C} \quad \text { and } \quad C=i \frac{\Delta^{1 / 2}-\mathbf{1}}{\Delta^{1 / 2}+\mathbf{1}} .
$$

REMARK 3.6. If $V$ is a standard subspace of $\mathcal{H}$ and $W \subseteq V+i V$ is a real subspace closed in $\mathcal{H}$ such that $W$ corresponds to a standard subspace of the complex Hilbert space $V_{\mathbb{C}}$, then $W$ is also standard in $\mathcal{H}$ because the closure of $W+i W$ contains $V+i V$, hence all of $\mathcal{H}$.

3.2. Symplectic aspects of standard subspaces. Let $V \subseteq \mathcal{H}$ be a standard subspace and consider the corresponding antiunitary representation $U^{V}: \mathbb{R}^{\times} \rightarrow \mathrm{AU}(\mathcal{H})$ with $U_{-1}^{V}=J^{V}$ and $U_{e^{t}}^{V}=\Delta^{-i t / 2 \pi}$ (Proposition $\left[3.2\right.$ ). Since the operators $\Delta^{i t}$ commute with $S=J \Delta^{1 / 2}$, they leave the closed subspace $V=\operatorname{Fix}(S)$ invariant. Further, the relation $J S J=\Delta^{1 / 2} J=S^{*}=J \Delta^{-1 / 2}$ implies that

$$
J V=V^{\prime}, \quad \text { where } \quad V^{\prime}:=\{w \in \mathcal{H}:(\forall v \in V) \operatorname{Im}\langle v, w\rangle=0\}=i V^{\perp_{\mathbb{R}}}
$$

is the symplectic orthogonal space of $V$, and $V^{\perp_{\mathbb{R}}}$ denotes the orthogonal complement of $V$ in the underlying real Hilbert space $\mathcal{H}^{\mathbb{R}}$ ([Lo08, Prop. 3.2]). In particular, the orbit $U_{\mathbb{R}^{\times}}^{V} V=\left\{V, V^{\prime}\right\}$ consists of at most two standard subspaces.

LEMMA 3.7. The following assertions hold:

(i) $U^{V^{\prime}}(t)=U^{V}\left(t^{-1}\right)$ for $t \in \mathbb{R}^{\times}$, is the antiunitary representation corresponding to $V^{\prime}$. 
(ii) $J_{V^{\prime}}=J_{V}$ and $\Delta_{V^{\prime}}=\Delta_{V}^{-1}$.

(iii) $V \cap V^{\prime}=\mathcal{H}^{U^{V}}$ is the fixed point space for the antiunitary representation $\left(U^{V}, \mathcal{H}\right)$ of $\mathbb{R}^{\times}$.

(iv) $V=V^{\prime}$ is equivalent to $\Delta=\mathbf{1}$.

Proof. (i) and (ii) follow immediately from $V^{\prime}=\operatorname{Fix}\left(J \Delta^{-1 / 2}\right)$.

(iii) If $v \in V \cap V^{\prime}$, then $v=S v=S^{*} v$ implies $\Delta v=v$ and hence $J v=v$. Conversely, these two relations imply $v \in V \cap V^{\prime}$.

(iv) follows from (ii).

REMARK 3.8. (Direct sums of standard subspaces)

(a) Suppose that $V_{j} \subseteq \mathcal{H}_{j}$ are standard subspaces for $j=1,2$. Then $V:=V_{1} \oplus V_{2} \subseteq$ $\mathcal{H}_{1} \oplus \mathcal{H}_{2}$ is a standard subspace. We have $J_{V}=J_{V_{1}} \oplus J_{V_{2}}$ and $\Delta_{V}=\Delta_{V_{1}} \oplus \Delta_{V_{2}}$. In particular, the corresponding antiunitary representation $U^{V}$ of $\mathbb{R}^{\times}$is the direct sum $U^{V_{1}} \oplus U^{V_{2}}$.

(b) In particular, every standard subspace $V$ can be written as such a direct sum

$$
V=\left(V \cap V^{\prime}\right) \oplus V_{1}, \quad \text { where } \quad V_{1}^{\prime} \cap V_{1}=\{0\}
$$

and $\left(V \cap V^{\prime}\right)_{\mathbb{C}}$ is the set of fixed points of the unitary representation $\left.U^{V}\right|_{\mathbb{R}_{+}^{\times}}$(Lemma 3.7).

LEMmA 3.9. Let $V$ be a standard subspace, $V_{1} \subseteq V$ be a closed subspace and $V_{2}:=V \cap V_{1}^{\perp_{\mathbb{R}}}$ be its orthogonal complement in $V$. Then the following are equivalent:

(i) $V=V_{1} \oplus V_{2}$ is a direct sum of standard subspaces.

(ii) $V_{1} \subseteq V_{2}^{\prime}$, i.e., $i V_{1} \perp V_{2}$ in $\mathcal{H}$.

(iii) $V_{1}$ is invariant under the modular automorphisms $\left(\Delta_{V}^{i t}\right)_{t \in \mathbb{R}}$.

If these conditions are satisfied and $V_{1}$ is also standard, then $V=V_{1}$.

Proof. (i) $\Leftrightarrow$ (ii) is easy to verify.

(i) $\Leftrightarrow$ (iii): Clearly, (i) implies (iii). To see the converse, consider the closed subspace $\mathcal{H}_{1}:=\overline{V_{1}+i V_{1}}$ of $\mathcal{H}$. Then, for each $v \in V$, the curve $t \mapsto \Delta_{V}^{-i t / 2 \pi} v$ is contained in $\mathcal{H}_{1}$, hence the same is true for its analytic continuation to the strip $\{z \in \mathbb{C}: 0 \leq \operatorname{Im} z \leq \pi\}$ (Remark 3.3). Therefore $\Delta^{1 / 2} v=J v \in \mathcal{H}_{1}$ and thus $\mathcal{H}_{1}$ is invariant under the antiunitary representation $U^{V}$ of $\mathbb{R}^{\times}$corresponding to $V$ (Proposition 3.2). Since the orthogonal decomposition $\mathcal{H}=\mathcal{H}_{1} \oplus \mathcal{H}_{1}^{\perp}$ reduces $U^{V}$, the standard subspace $V$ decomposes accordingly.

If (i)-(iii) are satisfied and $V_{1}$ is also standard, then (i) implies that $V_{1}=V$ (cf. Lo08, Prop. 3.10]).

3.3. Orthogonal real one-parameter groups. For any standard subspace $V$, the unitary operators $\Delta^{i t}$ define on the real Hilbert space $V$ a continuous orthogonal oneparameter group $(U, V)(\$ 3.2)$.

If, conversely, $\left(U_{t}\right)_{t \in \mathbb{R}}$ is a strongly continuous one-parameter group on the real Hilbert space $V$, then we can recover the corresponding embedding of $V$ as a standard subspace as follows. Let $V_{0}:=V^{U}$ be the subspace of $U$-fixed vectors and $V_{1}:=V_{0}^{\perp}$. Then $U_{t}=e^{t D}$ with a skew-symmetric infinitesimal generator $D=-D^{\top}$ satisfying $V_{0}=\operatorname{ker} D$. On $V_{1}$ we 
have the polar decomposition $D=I|D|$, where $I$ is a complex structure and $|D|=\sqrt{-D^{2}}$. We now consider the bounded skew-symmetric operator $C$ on $V$ defined by $\left.C\right|_{V_{0}}=0$ and

$$
\left.C\right|_{V_{1}}=I \frac{\mathbf{1}-e^{-|D|}}{\mathbf{1}+e^{-|D|}}
$$

Then $h(v, w):=(v, w)+i(v, C w)$ leads to an embedding of $V$ as a standard subspace $V \subseteq \mathcal{H}$ as in Remark 3.5 (a). The operator $D$ can be recovered directly from $C$ by $\left.D\right|_{V_{0}}=0$ and

$$
\left.D\right|_{V_{1}}=I \log \left(\frac{\mathbf{1}+\left|C_{1}\right|}{\mathbf{1}-\left|C_{1}\right|}\right)
$$

(cf. [NÓ16, Rem. 4.3], where different sign conventions are used).

The orthogonal one-parameter group $\left(U_{t}\right)_{t \in \mathbb{R}}$ on $V$ is trivial if and only if $D=0$, which corresponds to $\Delta=\mathbf{1}$, resp., to $C=0$, resp., to $V=\mathcal{H}^{J}$ (Lemma 3.7 (iv)).

3.4. Half-sided modular inclusions of standard subspaces. We have seen above that standard subspaces $V \subseteq \mathcal{H}$ are in one-to-one correspondence with antiunitary representations $U^{V}: \mathbb{R}^{\times} \rightarrow \mathrm{AU}(\mathcal{H})$ (Proposition 3.2). In this subsection we shall see how certain inclusions of standard subspaces can be related to antiunitary positive energy representations of $A f f(\mathbb{R})$ (cf. Section 2.4.1). Here the positive energy condition for the translation group turns out to be the crucial link between the inclusion order on $\operatorname{Stand}(\mathcal{H})$ and the affine geometry of the real line.

There are two ways to approach inclusions of standard subspaces. One is to consider the interaction of a unitary one-parameter group with a standard subspace, which leads to the concept of a Borchers pairs and the other considers the modular groups of two standard subspaces and leads to the concept of a half-sided modular inclusion. These perspectives have been introduced by Borchers ([Bo92 $)$ and Wiesbrock ( tively, in the context of von Neumann algebras (see $\$ 4.2$ for the translation to standard subspaces and [Lo08] for the results in the context of standard subspaces).

DEFINITION 3.10. (a) Let $\left(U_{t}\right)_{t \in \mathbb{R}}$ be a continuous unitary one-parameter group on $\mathcal{H}$ and $V \subseteq \mathcal{H}$ be a standard subspace. We call $(U, V)$ a (positive/negative) Borchers pair if $U_{t} V \subseteq V$ holds for $t \geq 0$ and $U_{t}=e^{i t P}$ with $\pm P \geq 0$.

(b) We call an inclusion $K \subseteq H$ of standard subspaces of $\mathcal{H}$ a \pm half-sided modular inclusion if

$$
\Delta_{H}^{-i t} K \subseteq K \quad \text { for } \quad \pm t \geq 0 .
$$

REMARK 3.11. The inclusion $K \subseteq H$ is positive half-sided modular if and only if the inclusion $H^{\prime} \subseteq K^{\prime}$ is negative half-sided modular ([Lo08, Cor. 3.23]).

The following theorem provides a passage from Borchers pairs to antiunitary representations of Aff $(\mathbb{R})$ ([BGL02, Thm. 3.2], [Lo08, Thm. 3.15]).

Theorem 3.12 (Borchers' Theorem-one particle case). If $(U, V)$ is a positive/negative Borchers pair, then

$$
U^{V}(a) U(b) U^{V}(a)^{-1}=U\left(a^{ \pm 1} b\right) \quad \text { for } \quad a \in \mathbb{R}^{\times}, b \in \mathbb{R},
$$

i.e., we obtain an antiunitary positive energy representation $(\widetilde{U}, \mathcal{H})$ of $\operatorname{Aff}(\mathbb{R})$ by $\widetilde{U}_{(b, a)}=$ $U(b) U^{V}(a)$. 
We are now ready to explain how inclusions of standard subspaces are related to antiunitary representations of $\operatorname{Aff}(\mathbb{R})$. The following result contains in particular a converse of Borchers' Theorem. For its formulation, we recall the one-to-one correspondence between standard subspaces and antiunitary representations of $\mathbb{R}^{\times}$from Proposition 3.2 . THEOREM 3.13. (Antiunitary positive energy representations of $\mathrm{Aff}(\mathbb{R})$ and standard subspaces) Let $(U, \mathcal{H})$ be an antiunitary representation of $\mathrm{Aff}(\mathbb{R})$. For each $x \in \mathbb{R}$, we consider the homomorphism

$$
\gamma_{x}: \mathbb{R}^{\times} \rightarrow \operatorname{Aff}(\mathbb{R}), \quad \gamma_{x}(s):=(x, 1)(0, s)(-x, 1)=((1-s) x, s)
$$

whose range is the stabilizer group $\operatorname{Aff}(\mathbb{R})_{x}$ and the corresponding family $\left(V_{x}\right)_{x \in \mathbb{R}}$ of standard subspaces determined by $U^{V_{x}}=U \circ \gamma_{x}$. Then the following assertions hold:

(i) $U_{(t, s)} V_{x}=V_{t+s x}$ and $U_{(t,-s)} V_{x}=V_{t-s x}^{\prime}$ for $t, x \in \mathbb{R}, s>0$.

(ii) The following are equivalent:

(a) $U$ is a positive energy representation.

(b) $V_{s} \subseteq V_{0}$ for $s \geq 0$.

(c) $V_{s} \subseteq V_{t}$ for $s \geq t$.

(d) $\left(W, V_{0}\right)$ with $W_{t}:=U_{(t, 1)}$ is a positive Borchers pair.

(e) $V_{1} \subseteq V_{0}$ is a thalf-sided modular inclusion.

(iii) $V_{x}=V_{0}$ for every $x \in \mathbb{R}$ is equivalent to $U_{(b, 1)}=\mathbf{1}$ for every $b \in \mathbb{R}$.

(iv) $V_{\infty}:=\bigcap_{t \in \mathbb{R}} V_{t}=\left\{v \in V_{0}:(\forall b \in \mathbb{R}) U_{(b, 1)} v=v\right\}$ is the fixed point space for the translations.

(v) $V_{0} \cap V_{0}^{\prime}=\mathcal{H}^{\operatorname{Aff}(\mathbb{R})}=\left\{v \in \mathcal{H}:(\forall g \in \operatorname{Aff}(\mathbb{R})) U_{g} v=v\right\}$.

Proof. (i) follows from $(t, s) \gamma_{x}(t, s)^{-1}=\gamma_{t+s x}, U_{(0,-1)} V_{0}=V_{0}^{\prime}$ and $V_{x}^{\prime}=U_{(x, 1)} V_{0}^{\prime}$.

(ii) (a) $\Leftrightarrow$ (b): For $W(s):=U_{(s, 1)}$ we have

$$
\Delta_{V_{0}}^{-i t / 2 \pi} W(s) \Delta_{V_{0}}^{i t / 2 \pi}=U_{\left(0, e^{t}\right)} W(s) U_{\left(0, e^{-t}\right)}=W\left(e^{t} s\right),
$$

so that the assertion follows from the converse of Borchers' Theorem [Lo08, Thm. 3.17].

(b) $\Leftrightarrow$ (c) follows from $V_{t}=W(t) V_{0}$ for $t \in \mathbb{R}$.

By definition, (d) is equivalent to (a) and (b).

(b) $\Leftrightarrow(\mathrm{e})$ : From (b) we derive (e) by

$$
U_{e^{t}}^{V_{0}} V_{1}=U_{\left(0, e^{t}\right)} V_{1}=V_{e^{t}}=U_{(1,1)} V_{e^{t}-1} \subseteq U_{(1,1)} V_{0}=V_{1} .
$$

From (e) we obtain, conversely, for $t \geq 0$

$$
U_{(1,1)} V_{0}=V_{1} \supseteq U_{e^{t}}^{V_{0}} V_{1}=V_{e^{t}}=U_{(1,1)} V_{e^{t}-1},
$$

and thus $V_{e^{t}-1} \subseteq V_{0}$, which implies (b).

(iii) If $W(x):=U_{(x, 1)}=\mathbf{1}$ for every $x \in \mathbb{R}$, then $V_{x}=W(x) V_{0}=V_{0}$. If, conversely, $W(x) V_{0}=V_{x}=V_{0}$ for every $x \in \mathbb{R}$, then every $W(x)$ commutes with $\Delta_{V_{0}}$ and $J_{V_{0}}$, so that Theorem 3.12 yields $W(x)=\mathbf{1}$ for every $x \in \mathbb{R}$.

(iv) By (i), the closed real subspace $V_{\infty}$ of $V_{0}$ is invariant under $\operatorname{Aff}(\mathbb{R})_{0}$. Hence Lemma 3.9 implies that it is a direct summand of the standard subspace $V_{0}$ and therefore also invariant under $J:=U_{(0,-1)}$. Now (iii) implies that the translation group fixes $V_{\infty}$ 
pointwise. Conversely, every fixed vector $v \in V_{0}$ of the translations is contained in each subspace $V_{x}=W(x) V_{0}$, hence also in $V_{\infty}$.

(v) From Lemma 3.7(iv) we know that $V_{0} \cap V_{0}^{\prime}$ is the space of fixed vector for the dilation group $\left(U_{(0, a)}\right)_{a \in \mathbb{R}^{\times}}$. Proposition 2.30(c) implies the translations also act trivially on this space. This proves (v).

REMARK 3.14. (a) If the momentum operator $P$ from Theorem 3.12 is strictly positive, then the space of fixed points for the dilation subgroup is trivial and Theorem 3.13 (iv) implies $V_{\infty}=\{0\}$.

(b) If $(U, V)$ is a Borchers pair for which $U_{t} V \subseteq V$ for all $t \in \mathbb{R}$, then $U_{t} V=V$ for every $t \in \mathbb{R}$ because $V=U_{0} V=U_{t} U_{-t} V \subseteq U_{t} V$. Now Theorem 3.13(iii) entails $U_{t}=\mathbf{1}$ for every $t \in \mathbb{R}$. Therefore non-trivial representations of the translation group lead to proper inclusions.

(c) For a Borchers pair $(U, V)$, the operators $\left(U_{t}\right)_{t \geq 0}$, and the modular operators $\left(\Delta^{i t}\right)_{t \in \mathbb{R}}$ act by isometries on the real Hilbert space $V$, so that we obtain a representation of the semigroup $[0, \infty) \rtimes \mathbb{R}_{+}^{\times}$by isometries on $V$. In this sense we may consider Borchers' Theorem 3.12 as a higher dimensional analog of the Lax-Phillips Theorem which provides a normal form for one-parameter semigroups of isometries on real Hilbert spaces as translations acting on spaces like $L^{2}\left(\mathbb{R}^{+}, \mathcal{K}\right)$, where $\mathcal{K}$ is a Hilbert space (cf. Remark 3.17 (b) and [NO15]).

The connection with the Lax-Phillips Theorem can also be made more direct as follows. The subspace $H:=U_{1} V$ is invariant under the modular automorphisms $\left(\Delta^{-i t}\right)_{t \geq 0}$. More precisely, $\Delta^{-i t} H=\Delta^{-i t} U_{1} V=U_{e^{2 \pi t}} V=V_{e^{2 \pi t}} \subseteq V_{1}=H$ for $t \geq 0$, in the notation of Theorem 3.13. This shows that $\bigcup_{t \in \mathbb{R}} \Delta^{-i t} H$ is dense in $V$ and that $\bigcap_{t>0} \Delta^{-i t} H=V_{\infty}$ is the fixed point set for $\left(U_{t}\right)_{t \in \mathbb{R}}$ in $V$ (Theorem 3.13 (iv)). Assuming that $U$ has no non-zero fixed vectors (as in (a) above), we obtain $V_{\infty}=\{0\}$. This means that the subspace $H \subseteq V$ is outgoing in the sense of Lax-Phillips for the orthogonal one-parameter $\operatorname{group}\left(\Delta^{-i t}\right)_{t \in \mathbb{R}}$.

The group $\operatorname{Aff}(\mathbb{R})$ is generated by translations and dilations, which is the structure underlying Borchers pairs. But we can also generate it by the subgroups $\gamma_{0}\left(\mathbb{R}^{\times}\right)$and $\gamma_{1}\left(\mathbb{R}^{\times}\right)$. For every antiunitary representation $(U, \mathcal{H})$ of $\operatorname{Aff}(\mathbb{R})$, the corresponding modular objects lead to two standard subspaces $V_{0}$ and $V_{1}$ and we have already seen above that $V_{1} \subseteq V_{0}$ is a positive half-sided modular inclusion if $U$ is of positive energy. The following theorem provides a converse (see [Lo08, Thm. 3.21]).

ThEOREM 3.15 (Wiesbrock Theorem-one particle case). An inclusion $K \subseteq H$ of standard subspaces is positive half-sided modular if and only if there exists an antiunitary positive energy representation $(U, \mathcal{H})$ of $\operatorname{Aff}(\mathbb{R})$ with $K=V_{1}$ and $H=V_{0}$.

Proof. In view of Theorem 3.13(ii)(e), it remains to show the existence of $U$ if the inclusion is thalf-sided modular. In view of [Lo08, Thm. 3.21], there exists a unitary positive energy representation $(U, \mathcal{H})$ of the connected affine group $\operatorname{Aff}(\mathbb{R})_{0}$ such that $U_{\gamma_{0}\left(e^{t}\right)}=U^{H}\left(e^{t}\right)$ and $U_{\gamma_{1}\left(e^{t}\right)}=U^{K}\left(e^{t}\right)$ for $t \in \mathbb{R}$. Further, the translation unitaries $W_{t}:=U_{(t, 1)}$ satisfy $W_{1} H=K$ and $W_{t} H \subseteq H$ for $t \geq 0$. Therefore $(W, H)$ is a Borchers pair, and thus $\widetilde{U}_{(b, a)}:=W_{b} U_{a}^{H}$ defined an extension of $U$ to an antiunitary representation 
of $\operatorname{Aff}(\mathbb{R})$ (Theorem $\underline{3.12}$ ). The corresponding subspaces are $V_{0}=H$ by construction, and $V_{1}=W_{1} V_{0}=W_{1} H=K$.

EXAMPLES 3.16. Below we provide an explicit description of a positive Borchers pair in a concrete model of the irreducible antiunitary positive energy representation of Aff $(\mathbb{R})$ (cf. Theorem 3.12 and [LL14, $\S 4]$ ). A slight variation of (6) leads to the antiunitary representation of $\operatorname{Aff}(\mathbb{R})$ on $L^{2}\left(\mathbb{R}_{+}, \frac{d p}{p}\right)$ by

$$
\left(U_{\left(b, e^{t}\right)} \psi\right)(p)=e^{i b p} \psi\left(e^{t} p\right), \quad\left(U_{(0,-1)} \psi\right)(p)=\overline{\psi(p)} .
$$

Transforming it with the unitary operator $\Gamma: L^{2}\left(\mathbb{R}_{+}, \frac{d p}{p}\right) \rightarrow L^{2}(\mathbb{R}, d \theta), \Gamma(\psi)(\theta)=\psi\left(e^{\theta}\right)$, transforms it into the representation

$$
\left(U_{\left(b, e^{t}\right)} \psi\right)(\theta):=e^{i b e^{\theta}} \psi(\theta+t), \quad\left(U_{(0,-1)} \psi\right)(\theta):=\overline{\psi(\theta)} .
$$

On the $\operatorname{strip} \mathcal{S}_{\pi}:=\{z \in \mathbb{C}: 0<\operatorname{Im} z<\pi\}$ we have the Hardy space

$$
\mathcal{H}^{2}\left(\mathcal{S}_{\pi}\right):=\left\{\psi \in \mathcal{O}\left(\mathcal{S}_{\pi}\right): \sup _{0<\lambda<\pi} \int_{\mathbb{R}}|\psi(\theta+i \lambda)|^{2} d \theta<\infty\right\},
$$

and in these terms, the standard subspace $V_{0}$ corresponding to $\gamma_{0}(t)=(0, t)$ is given by

$$
V_{0}=\left\{\psi \in \mathcal{H}^{2}\left(\mathcal{S}_{\pi}\right):\left(\forall z \in \mathcal{S}_{\pi}\right) \overline{\psi(i \pi+\bar{z})}=\psi(z)\right\}
$$

On the strip $\mathcal{S}_{\pi}$, the functions $B(z):=e^{i b e^{z}}$ satisfy

$$
|B(x+i y)|=e^{-b \operatorname{Im}\left(e^{x+i y}\right)}=e^{-b e^{x} \sin y} \leq 1 \quad \text { because } \quad \sin y \geq 0
$$

and $\overline{B(i \pi+\bar{z})}=B(z)$. This shows hat, for $b \geq 0$, multiplication with $B$ defines an isometry of the Hardy space $\mathcal{H}^{2}\left(\mathcal{S}_{\pi}\right)$ and also of the real subspace $V_{0}$ into itself (cf. Remark 3.14(c)). One can show that all unitary operators commuting with the representation of the one-parameter group $\left(U_{(b, 1)}\right)_{b \in \mathbb{R}}$ and mapping $V_{0}$ into itself are multiplications with bounded holomorphic functions $\varphi$ on $\mathcal{S}_{\pi}$ satisfying $\varphi(i \pi+\bar{z})=\overline{\varphi(z)}$ and whose boundary values in $L^{\infty}(\mathbb{R}, \mathbb{C}$ ) satisfy $|\varphi(x)|=1$ for almost every $x \in \mathbb{R}$ (cf. Re$\operatorname{mark} 4.19(\mathrm{c}))$.

For explicit descriptions of standard subspaces related to free fields, we refer to FG89, p. $422 \mathrm{ff}]$.

REMARK 3.17. (a) If $K \subseteq H$ is a proper positive half-sided modular inclusion and $V$ is a closed real subspace with $K \subseteq V \subseteq H$, then $V$ is clearly standard. However, neither the inclusion $K \subseteq V$ nor the inclusion $V \subseteq H$ has to be half-sided modular. In fact, the existence of the unitary one-parameter group $\left(U_{t}\right)_{t \in \mathbb{R}}$ with $U_{1} H=K$ implies that all the inclusions $U_{t} H \subseteq U_{s} H$ for $0 \leq s<t \leq 1$ are proper (Theorem 3.13(ii)). Therefore $K$ has infinite codimension in $H$. So subspaces $V$ for which $V / K$ or $H / V$ is finite dimensional yield counterexamples.

(b) Let $V$ be a standard subspace. We write $\operatorname{hsm}_{+}(V)$ for the set of all standard subspaces $H \subseteq V$ for which the inclusion $H \subseteq V$ is positive half-sided modular. To obtain a description of this set, one can proceed as follows. First we can split off the maximal direct summand $H_{1}:=\bigcap_{t \in \mathbb{R}} \Delta_{V}^{i t} H$ of $V$ contained in $H$ (Lemma 3.9). This leaves us with the situation where $H_{1}=\{0\}$. 
Decomposition of the corresponding antiunitary representation $(U, \mathcal{H})$ of $\operatorname{Aff}(\mathbb{R})$ (Theorem 3.15 into a subspaces $\mathcal{H}^{0}$ on which the translations act trivially and an orthogonal space $\mathcal{H}^{+}$on which the representation is of strict positive energy, we accordingly obtain the direct sum $V=V^{0} \oplus V^{+}$of standard subspaces and $H=V^{0} \oplus H^{+}$. Hence our assumption implies $V^{0}=\{0\}$ and $\mathcal{H}=\mathcal{H}^{+}$. Now Lo08, Thm. 2.8] implies that $U$ is a multiple of the unique irreducible positive energy representation of $\operatorname{Aff}(\mathbb{R})$, so that we may assume that

$$
\mathcal{H}=L^{2}\left(\mathbb{R}_{+}, \mathcal{K}\right) \quad \text { and } \quad\left(U_{\left(t, e^{s}\right)} f\right)(x)=e^{i t x} e^{s / 2} f\left(e^{s} x\right), \quad U_{(0,-1)} f=J_{K} f,
$$

where $J_{K}$ is a conjugation on $\mathcal{K}$ (see 2.4 .1 ).

As all antiunitary rerpresentations of $\mathrm{Aff}(\mathbb{R})$ with strictly positive energy and the same multiplicity are equivalent, we obtain all such standard subspaces $H$ by applying elements of the group

$$
\begin{aligned}
K & :=\{U \in \mathrm{U}(\mathcal{H}): U V=V\}=\left\{U \in \mathrm{U}(\mathcal{H}):\left(\forall a \in \mathbb{R}^{\times}\right) U U_{(0, a)} U^{-1}=U_{(0, a)}\right\} \\
& \cong\left\{U \in \mathrm{O}(V):\left.\left(\forall a \in \mathbb{R}^{\times}\right) U \Delta_{V}^{i t}\right|_{V}=\Delta_{V}^{i t} U\right\} .
\end{aligned}
$$

If $\mathcal{K}=\mathbb{C}$, then the representation of $\mathbb{R}^{\times}$on $\mathcal{H}$ is (by Fourier transform) equivalent to the representation of $\mathbb{R}$ on $L^{2}(\mathbb{R}, \mathcal{K})$ by

$$
\left(V_{e^{x}} \xi\right)(p)=e^{i x p} \xi(p) \quad \text { and } \quad\left(V_{-1} \xi\right)(p)=\overline{\xi(-p)} .
$$

Therefore any unitary operator $M$ on $\mathcal{H}$ commuting with $V_{\mathbb{R}^{\times}}$is of the form $(M \xi)(p)=$ $m(p) \xi(p)$, where $m: \mathbb{R} \rightarrow \mathbb{T}$ is a measurable function satisfying $m(-p)=\overline{m(p)}$. It would be interesting to see how this relates to the inner functions corresponding to endomorphisms of one-dimensional standard pairs (see Remark 4.19(c)).

Combining the preceding results with the fact that the infinite dimensional irreducible positive energy representation of $\operatorname{Aff}(\mathbb{R})_{0}$ extends to an antiunitary positive energy representation of $\mathrm{PGL}_{2}(\mathbb{R})$ with lowest weight 1 (Theorem 2.34 and Corollary 2.32), we obtain ([Lo08, Cor. 4.15]):

THEOREM 3.18. There exists a one-to-one correspondence between

(i) Positive half-sided modular inclusions $K \subseteq H$.

(ii) Antiunitary positive energy representations of $\mathrm{Aff}(\mathbb{R})$.

(iii) Positive Borchers pairs $(V, U)$.

(iv) Unitary representations of $\mathrm{PSL}_{2}(\mathbb{R})$ which are direct sums of representations with lowest weights 0 or 1 .

An important aspect of the last item in the preceding theorem is that it leads to a considerable enrichment of the geometry. Starting with a positive half-sided modular inclusion $K \subseteq H$, we obtain an antiunitary representation of $\mathrm{PGL}_{2}(\mathbb{R})$. Accordingly, for every interval $I \subseteq \mathbb{S}^{1}$, the corresponding homomorphism $\gamma^{I}: \mathbb{R}^{\times} \rightarrow \mathrm{PGL}_{2}(\mathbb{R})$ (Example 2.28) determines a standard subspace $V_{I}$, whereas the representation of Aff( $(\mathbb{R})$ only leads to standard subspaces $V_{I}$ indexed by the open half-lines $I \subseteq \mathbb{R}$.

The following theorem is another result in this direction. It relates pairs of halfsided modular inclusions via the corresponding antiunitary representations of Aff $(\mathbb{R})$ to representations of the two-dimensional Poincaré group $P(2)_{+}$, resp., $\operatorname{PGL}_{2}(\mathbb{R})$. 
THEOREM 3.19. (a) Let $H_{1} \subseteq V$ be a-half sided modular inclusion and $H_{2} \subseteq V$ be a +half sided modular inclusion such that

$$
J_{H_{1}} J_{H_{2}}=J_{V} J_{H_{2}} J_{H_{1}} J_{V} \text {. }
$$

Then the corresponding three modular one-parameter groups combine to a faithful continuous antiunitary representation of the proper Poincaré group $P(2)_{+} \cong \mathbb{R}^{1,1} \rtimes \mathrm{SO}_{1,1}(\mathbb{R})$.

(b) Let $H, V$ be standard subspaces of $\mathcal{H}$ such that $H \cap V \subseteq V$ and $H \cap V \subseteq H$ are -, resp., +half-sided modular inclusions satisfying $J_{H} V=V$. Then the corresponding three representation $U^{H}, U^{V}$ and $U^{H \cap V}$ generate a faithful antiunitary positive energy representation of $\mathrm{PGL}_{2}(\mathbb{R})$.

Proof. (a) The version for von Neumann algebras is contained in [Wi98, Lemma 10] and we shall see in 4.2 below how the present version follows from this one. Here are some comments on the proof. Clearly, the two half-sided modular inclusion defines two antiunitary representations $U^{1 / 2}$ of $\operatorname{Aff}(\mathbb{R}) \cong \mathbb{R} \rtimes \mathbb{R}^{\times}$that coincide on the subgroup of dilations. Now the main point is to verify that the corresponding images of the translation groups commute.

That (10) is necessary can be seen as follows. If we have a unitary representation of $P(2)_{0}$ as required, then

$$
U_{\left(b_{1}, b_{2}, e^{t}\right)}:=U_{b_{1}}^{1} U_{b_{2}}^{2} \Delta_{V}^{-i t / 2 \pi} \quad \text { and } \quad U_{(0,0,-1)}:=J_{V}
$$

defines an extension to an antiunitary representation of $P(2)_{+}$. Here the modular conjugations

$$
J_{H_{1}}=U_{(2,0,-1)} \quad \text { and } \quad J_{H_{2}}=U_{(0,2,-1)}
$$

satisfy (10) because $(0,0,-1)(0,2,-1)(2,0,-1)(0,0,-1)=(2,-2,1)$ holds in $P(2)$.

(b) The von Neumann version is Wi93b, Thm. 3] (see also [Wi93c, Lemma 2]).

\subsection{Half-sided modular intersections.}

Definition 3.20. We consider two standard subspaces $H_{1}, H_{2} \subseteq \mathcal{H}$ and their modular objects $\left(\Delta_{H_{j}}, J_{H_{j}}\right)_{j=1,2}$. We say that the pair $\left(H_{1}, H_{2}\right)$ has a \pm modular intersection if the following two conditions are satisfied

(MI1) The intersection $H_{1} \cap H_{2}$ is a standard subspace and the inclusions $H_{1} \cap H_{2} \subseteq H_{j}$, $j=1,2$, are \pm half-sided modular.

(MI2) The strong limit $S:=\lim _{t \rightarrow \pm \infty} \Delta_{H_{1}}^{i t} \Delta_{H_{2}}^{-i t}$ (which always exists by Remark 3.21 below) satisfies $J_{H_{1}} S J_{H_{1}}=S^{-1}$.

REMARK 3.21. (a) In Aff $(\mathbb{R})$ the two multiplicative one-parameter groups $\gamma_{0}(r):=(0, r)$ and $\gamma_{1}(r):=(1-r, r)$ (stabilizing the points 0 and 1 , resp.) satisfy

$$
\gamma_{0}(r) \gamma_{1}\left(r^{-1}\right)=(0, r)\left(1-r^{-1}, r^{-1}\right)=(r-1,1),
$$

so that $\lim _{r \rightarrow 0} \gamma_{0}(r) \gamma_{1}\left(r^{-1}\right)=(-1,1)$ exists. As a consequence, for every continuous unitary representation $(U, \mathcal{H})$ of $\operatorname{Aff}(\mathbb{R})_{0}$, the limit

$$
\lim _{r \rightarrow 0} U_{\gamma_{0}(r)} U_{\gamma_{1}(r)}^{-1}=U_{(-1,1)}
$$

exists. 
(b) Suppose that the +-variant of (MI1) is satisfied and let $\left(U^{1}, \mathcal{H}\right),\left(U^{2}, \mathcal{H}\right)$ be the corresponding positive energy representations of $\operatorname{Aff}(\mathbb{R})$ satisfying

$$
H_{1} \cap H_{2}=V_{1}^{1}=V_{1}^{2}, \quad H_{1}=V_{0}^{1} \quad \text { and } \quad H_{2}=V_{0}^{2},
$$

where $\left(V_{x}^{j}\right)_{x \in \mathbb{R}}$ are the corresponding families of standard subspaces (Theorem 3.13). We write $W^{j}(t):=U_{(t, 1)}^{j}$ for the representations of the translation group. Then (a) implies that

$$
W^{1}(-1)=U_{(-1,1)}^{1}=\lim _{t \rightarrow \infty} U_{\gamma_{0}\left(e^{-t}\right)}^{1} U_{\gamma_{1}\left(e^{t}\right)}^{1}=\lim _{t \rightarrow \infty} \Delta_{H_{1}}^{i t / 2 \pi} \Delta_{H_{1} \cap H_{2}}^{-i t / 2 \pi}=\lim _{t \rightarrow \infty} \Delta_{H_{1}}^{i t} \Delta_{H_{1} \cap H_{2}}^{-i t}
$$

and likewise $W^{2}(-1)=\lim _{t \rightarrow \infty} \Delta_{H_{2}}^{i t} \Delta_{H_{1} \cap H_{2}}^{-i t}$. This leads to

$$
S:=\lim _{t \rightarrow \infty} \Delta_{H_{1}}^{i t} \Delta_{H_{2}}^{-i t}=W^{1}(-1) W^{2}(1),
$$

so that the limit in (MI2) exists whenever (MI1) is satisfied. From the relation

$$
S H_{2}=W^{1}(-1) W^{2}(1) H_{2}=W^{1}(-1)\left(H_{1} \cap H_{2}\right)=H_{1}
$$

it follows that $S J_{H_{2}} S^{-1}=J_{H_{1}}$, i.e.,

$$
S J_{H_{2}}=J_{H_{1}} S \text {. }
$$

As condition (MI2) means that $J_{H_{1}} S$ is an involution, and this is equivalent to $S J_{H_{1}}=$ $S\left(J_{H_{1}} S\right) S^{-1}$ being an involution, (14) shows that (MI2) is equivalent to the relation $J_{H_{2}} S J_{H_{2}}=S^{-1}$.

(c) If the negative variant of (MI1) is satisfied, then $H_{j}^{\prime} \subseteq\left(H_{1} \cap H_{2}\right)^{\prime}$ are +half-sided modular inclusions by Remark 3.11 Let $\left(U^{1}, \mathcal{H}\right),\left(U^{2}, \mathcal{H}\right)$ be the corresponding positive energy representations of Aff $(\mathbb{R})$ satisfying

$$
\left(H_{1} \cap H_{2}\right)^{\prime}=V_{0}^{1}=V_{0}^{2}, \quad H_{1}^{\prime}=V_{1}^{1} \quad \text { and } \quad H_{2}^{\prime}=V_{1}^{2}
$$

(Theorem 3.13). With the same notation as in (b), we obtain

$$
W^{1}(1)=\lim _{t \rightarrow \infty} U_{\gamma_{1}\left(e^{-t}\right)}^{1} U_{\gamma_{0}\left(e^{t}\right)}^{1}=\lim _{t \rightarrow-\infty} \Delta_{H_{1}^{\prime}}^{-i t / 2 \pi} \Delta_{\left(H_{1} \cap H_{2}\right)^{\prime}}^{i t / 2 \pi}=\lim _{t \rightarrow-\infty} \Delta_{H_{1}}^{i t} \Delta_{H_{1} \cap H_{2}}^{-i t}
$$

and likewise $W^{2}(1)=\lim _{t \rightarrow-\infty} \Delta_{H_{2}}^{i t} \Delta_{H_{1} \cap H_{2}}^{-i t}$. This leads to

$$
S:=\lim _{t \rightarrow-\infty} \Delta_{H_{1}}^{i t} \Delta_{H_{2}}^{-i t}=W^{1}(1) W^{2}(-1),
$$

so that the limit in (MI2) exists. Here $S H_{2}^{\prime}=H_{1}^{\prime}$ shows that (MI2) is equivalent to $J_{H_{2}} S J_{H_{2}}=S^{-1}$.

The following theorem extends Wiesbrock's Theorem 3.15 from half-sided modular inclusions to general modular intersections.

THEOREM 3.22 (Wiesbrock's Theorem for modular intersections - one particle version). For a pair $\left(H_{1}, H_{2}\right)$ of standard subspaces, the following assertions hold:

(a) $\left(H_{1}, H_{2}\right)$ has a +modular intersection if and only if there exists an antiunitary representation $(U, \mathcal{H})$ of $\mathrm{Aff}(\mathbb{R})$ such that the corresponding family of standard subspaces $\left(V_{x}\right)_{x \in \mathbb{R}}$ from Theorem 3.15 satisfies $V_{0}=H_{1}$ and $V_{1}=H_{2}$.

(b) $\left(H_{1}, H_{2}\right)$ has a-modular intersection if and only if there exists an antiunitary representation $(U, \mathcal{H})$ of $\operatorname{Aff}(\mathbb{R})$ such that $V_{0}=H_{1}^{\prime}$ and $V_{1}=H_{2}^{\prime}$. 
Proof. (a) Suppose first that $\left(H_{1}, H_{2}\right)$ has the +modular intersection property. In the context of Remark 3.21 (b) we then have

$$
J_{H_{1}} J_{H_{2}}=J_{H_{1}} J_{H_{1} \cap H_{2}} J_{H_{1} \cap H_{2}} J_{H_{2}}=U_{(0,-1)}^{1} U_{(2,-1)}^{1} U_{(2,-1)}^{2} U_{(0,-1)}^{2}=W^{1}(-2) W^{2}(2),
$$

and this operator commutes with $S$ by (MI2). From these relations Wiesbrock derives in Wi97 that the two one-parameter groups $W^{1}$ and $W^{2}$ commute, so that $W(t):=$ $W^{1}(t) W^{2}(-t)$ defines a unitary one-parameter group and $U_{(b, a)}:=W(b) U_{\gamma_{0}(a)}^{1}$ defines an antiunitary representation of $\mathrm{Aff}(\mathbb{R})$ for which the corresponding standard subspaces $\left(V_{x}\right)_{x \in \mathbb{R}}$ satisfy $V_{0}=H_{1}$ and $V_{1}=W(1) V_{0}=S^{-1} H_{1}=H_{2} .$.

Suppose, conversely, that $(U, \mathcal{H})$ is an antiunitary representation of $\operatorname{Aff}(\mathbb{R})$ and let $\left(V_{x}\right)_{x \in \mathbb{R}}$ be the corresponding family of standard subspaces. We decompose $(U, \mathcal{H})$ as a direct sum

$$
(U, \mathcal{H})=\left(U^{+}, \mathcal{H}^{+}\right) \oplus\left(U^{0}, \mathcal{H}^{0}\right) \oplus\left(U^{-}, \mathcal{H}^{-}\right),
$$

where the representation $U^{+}$has strictly positive energy, $U^{-}$has strictly negative energy and the translation group acts trivially on $\mathcal{H}^{0}$. Then the subspaces $V_{x}$ decompose accordingly as orthogonal direct sums $V_{x}=V_{x}^{+} \oplus V_{x}^{0} \oplus V_{x}^{-}$, where $V_{x}^{0}=V_{0}^{0}$ does not depend on $x$.

Theorem 3.13 now implies that $V_{x}^{ \pm} \subseteq V_{y}^{ \pm}$for $\pm(x-y) \geq 0$. To see that $V_{0}$ and $V_{1}$ have a +modular intersection, we first observe that

$$
V_{0} \cap V_{1}=\left(V_{0}^{+} \cap V_{1}^{+}\right) \oplus V_{0}^{0} \oplus\left(V_{0}^{-} \cap V_{1}^{-}\right)=V_{1}^{+} \oplus V_{0}^{0} \oplus V_{0}^{-}
$$

is an orthogonal direct sum of three standard subspaces, hence a standard subspace. Its invariance under the modular operators $\Delta_{V_{0}}^{-i t / 2 \pi}=U_{\gamma_{0}\left(e^{t}\right)}$ for $t \geq 0$ follows from the invariance of $V_{1}^{+}$under $U_{\gamma_{0}\left(e^{t}\right)}^{+}$and the invariance of $V_{0}^{+}$and $V_{0}^{0}$ under the corresponding modular group.

For the invariance of $V_{0} \cap V_{1}$ under $\Delta_{V_{1}}^{-i t / 2 \pi}=U_{\gamma_{1}\left(e^{t}\right)}=U_{\left(\left(1-e^{t}, e^{t}\right)\right.}$, we likewise use that

$$
U_{\left(1-e^{t}, e^{t}\right)}^{-} V_{0}^{-}=U_{\left(1-e^{t}, 1\right)}^{-} V_{0}^{-}=V_{1-e^{t}}^{-} \subseteq V_{0}^{-} \quad \text { for } \quad t \geq 0 .
$$

This shows that $\left(V_{0}, V_{1}\right)$ has a +modular intersection.

(b) If $\left(H_{1}, H_{2}\right)$ is a -modular intersection, we likewise obtain with Remark 3.21(c) that $U_{(b, a)}:=W(b) U_{\gamma_{1}(a)}^{1}$ and $W(t):=W^{1}(t) W^{2}(-t)$ define an antiunitary representation of Aff $(\mathbb{R})$ with $S=W(-1), V_{0}=V_{1}^{1}=H_{1}^{\prime}$ and $V_{1}=W(1) V_{0}=S^{-1} H_{1}^{\prime}=H_{2}^{\prime}$.

Suppose, conversely, that $(U, \mathcal{H})$ is an antiunitary representation of $\operatorname{Aff}(\mathbb{R})$. We use the notation from (a). To see that $V_{0}^{\prime}$ and $V_{1}^{\prime}$ have a -modular intersection, we first observe that

$$
V_{0}^{\prime} \cap V_{1}^{\prime}=\left(\left(V_{0}^{+}\right)^{\prime} \cap\left(V_{1}^{+}\right)^{\prime}\right) \oplus\left(V_{0}^{0}\right)^{\prime} \oplus\left(\left(V_{0}^{-}\right)^{\prime} \cap\left(V_{1}^{-}\right)^{\prime}\right)=\left(V_{0}^{+}\right)^{\prime} \oplus V_{0}^{0} \oplus\left(V_{1}^{-}\right)^{\prime}
$$

to see that $V_{0}^{\prime} \cap V_{1}^{\prime}$ is standard. Its invariance under the modular operators $\Delta_{V_{0}^{\prime}}^{i t / 2 \pi}=$ $\Delta_{V_{0}}^{-i t / 2 \pi}=U_{\gamma_{0}\left(e^{t}\right)}$ for $t \geq 0$ follows from the invariance of $\left(V_{1}^{-}\right)^{\prime}$ under $U_{\gamma_{0}\left(e^{t}\right)}^{-}$(Remark 3.11) and the invariance of $V_{0}^{+}$and $V_{0}^{0}$ under the corresponding modular group.

For the invariance of $V_{0}^{\prime} \cap V_{1}^{\prime}$ under $\Delta_{V_{1}^{\prime}}^{i t / 2 \pi}=U_{\gamma_{1}\left(e^{t}\right)}=U_{\left(1-e^{t}, e^{t}\right)}$, we likewise use that

$$
U_{\left(1-e^{t}, e^{t}\right)}^{+}\left(V_{0}^{+}\right)^{\prime}=U_{\left(1-e^{t}, 1\right)}^{+}\left(V_{0}^{+}\right)^{\prime}=\left(V_{1-e^{t}}^{+}\right)^{\prime} \subseteq\left(V_{0}^{+}\right)^{\prime} \quad \text { for } \quad t \geq 0 .
$$


Therefore $\left(V_{0}^{\prime}, V_{1}^{\prime}\right)$ has a -modular intersection.

The key point of modular intersections is that they no longer require any spectral condition on the corresponding representations of $\operatorname{Aff}(\mathbb{R})$. The preceding theorem shows that \pm modular intersections are characterized as pairs of standard subspaces that can be obtained from arbitrary antiunitary representations of $\operatorname{Aff}(\mathbb{R})$. This is of particular relevance for representations of Lorentz groups $\mathrm{SO}_{1, d-1}(\mathbb{R})$ which, for $d>3$, never satisfy any positive energy condition.

With the same method that we used to obtain Theorem 3.19, we now obtain by transcribing [Wi97, Thm. 6] from the context of von Neumann algebras the following theorem.

THEOREM 3.23. Let $H_{1}, H_{2}, H_{3}$ be three standard subspaces such that $\left(H_{1}, H_{2}\right)$ and $\left(H_{3}, H_{1}^{\prime}\right)$ are - modular intersections and $\left(H_{2}, H_{3}\right)$ is a +modular intersection. Then the corresponding antiunitary representations $U^{H_{j}}, j=1,2,3$, of $\mathbb{R}^{\times}$generate an anti-unitary representation of $\mathrm{PGL}_{2}(\mathbb{R})$.

Proof. For the proof we only has to observe that the group $G$ generated by the three modular one-parameter groups $\left(\Delta_{H_{j}}^{i t}\right)_{t \in \mathbb{R}}, j=1,2,3$ is invariant under $J_{H_{1}}$. For the subgroup $G_{12}$ generated by the operators $\Delta_{H_{1}}^{i t}$ and $\Delta_{H_{2}}^{i s}$, this follows from Theorem 3.15 and we likewise obtain the invariance of the subgroup $G_{13}$ generated by the operators $\Delta_{H_{1}}^{i t}$ and $\Delta_{H_{3}}^{i s}$. As $G$ is generated by $G_{12} \cup G_{13}$, the assertion follows.

4. A glimpse of modular theory. We now recall some of the key features of TomitaTakesaki Theory. In $\$ 4.2$ we discuss the translation between pairs $(\mathcal{M}, \Omega)$ of von Neumann algebras with cyclic separating vectors and standard subspaces $V$. More specifically, we discuss this translation for half-sided modular inclusions in $\$ 4.3$ and in $\$ 4.4$ we take a closer look at the space of modular conjugations of a von Neumann algebra.

4.1. The Tomita-Takesaki Theorem. Let $\mathcal{H}$ be a Hilbert space and $\mathcal{M} \subseteq B(\mathcal{H})$ be a von Neumann algebra. We call a unit vector $\Omega \in \mathcal{H}$

- cyclic if $\mathcal{M} \Omega$ is dense in $\mathcal{H}$.

- separating if the map $\mathcal{M} \rightarrow \mathcal{H}, M \mapsto M \Omega$ is injective.

It is easy to see that $\Omega$ is separating if and only if it is cyclic for the commutant $\mathcal{M}^{\prime}$.

Definition 4.1. We write $\operatorname{cs}(\mathcal{M})$ for the set of cyclic and separating unit vectors for $\mathcal{M}$.

TheOrem 4.2 (Tomita-Takesaki Theorem). Let $\mathcal{M} \subseteq B(\mathcal{H})$ be a von Neumann algebra and $\Omega \in \mathcal{H}$ be a cyclic separating vector for $\mathcal{M}$. Write $\mathcal{M}_{h}:=\left\{M \in \mathcal{M}: M^{*}=M\right\}$ for the real subspace of hermitian elements in $\mathcal{M}$. Then $V:=\overline{\mathcal{M}_{h} \Omega}$ is a standard subspace. The corresponding modular objects $(\Delta, J)$ satisfy

(a) $J \mathcal{M} J=\mathcal{M}^{\prime}$ and $\Delta^{i t} \mathcal{M} \Delta^{-i t}=\mathcal{M}$ for $t \in \mathbb{R}$.

(b) $J \Omega=\Omega, \Delta \Omega=\Omega$ and $\Delta^{i t} \Omega=\Omega$ for all $t \in \mathbb{R}$.

(c) For $M \in \mathcal{M} \cap \mathcal{M}^{\prime}$, we have $J M J=M^{*}$ and $\Delta^{i t} M \Delta^{-i t}=M$ for $t \in \mathbb{R}$.

Proof. We only show that $V$ is a standard subspace and refer to BR87, Thm. 2.5.14] for the other assertions. Clearly, $V$ is a closed real subspace for which $V+i V$ is dense 
because it contains $\mathcal{M}_{h} \Omega+i \mathcal{M}_{h} \Omega=\mathcal{M} \Omega$. The same holds for $W:=\overline{\mathcal{M}_{h}^{\prime} \Omega}$ because $\Omega$ is also cyclic for $\mathcal{M}^{\prime}$. For $M \in \mathcal{M}_{h}$ and $M^{\prime} \in \mathcal{M}_{h}^{\prime}$, we have

$$
\left\langle M \Omega, M^{\prime} \Omega\right\rangle=\left\langle M^{\prime} M \Omega, \Omega\right\rangle=\left\langle M M^{\prime} \Omega, \Omega\right\rangle=\left\langle M^{\prime} \Omega, M \Omega\right\rangle \in \mathbb{R},
$$

which implies that $\omega(V, W)=\{0\}$. Therefore $V \cap i V$ is a complex subspace of $W^{\perp}=\{0\}$, hence trivial. Now the main point is to show that the modular objects $(\Delta, J)$ associated to $V$ satisfy (a)-(c).

The key point of the Tomita-Takesaki Theorem is that it provides for each cyclic separating vector $\Omega \in \operatorname{cs}(\mathcal{M})$ a pair $(\Delta, J)$ of modular objects. The modular operators $\Delta$ and their spectra are the key tool in the classification of factors and in the characterization of von Neumann algebras by their natural cones by A. Connes Co73, Co74]. Here we emphasize that $(\Delta, J)$ is encoded in an antiunitary representation $U^{V}$ of $\mathbb{R}^{\times}$.

We first take a closer look at the antiunitary operators that come directly from $\mathcal{M}$ and its commutant. The picture will be refined in $\$ 4.4$ below.

EXAmPLE 4.3. Let $\mathcal{M} \subseteq B(\mathcal{H})$ be a von Neumann algebra, $G_{1}:=\mathrm{U}(\mathcal{M}) \times \mathrm{U}(\mathcal{M})$ and $\tau \in \operatorname{Aut}\left(G_{1}\right)$ be the flip automorphism. We consider the group $G:=G_{1} \rtimes\{\mathbf{1}, \tau\}$.

If $\Omega$ is a cyclic separating vector for $\mathcal{M}$ and $J$ the corresponding modular involution, then

$$
U_{\left(g, h, \tau^{\varepsilon}\right)}:=g J h J J^{\varepsilon}
$$

defines an antiunitary representation of the pair $\left(G, G_{1}\right)$.

Any other conjugation $\widetilde{J}$ on $\mathcal{H}$ that we can use to extend the unitary representation $\left.U\right|_{G_{1}}$ is of the form $\widetilde{J}=J g$ for some central unitary element $g \in \mathrm{U}\left(\mathcal{M} \cap \mathcal{M}^{\prime}\right)$.

Definition 4.4. A von Neumann algebra $\mathcal{M} \subseteq B(\mathcal{H})$ is said to be in symmetric form if there exists a conjugation $J$ on $\mathcal{H}$ with

$$
J \mathcal{M} J=\mathcal{M}^{\prime} \quad \text { and } \quad J Z J=Z^{*} \quad \text { for } \quad Z \in \mathcal{M} \cap \mathcal{M}^{\prime} .
$$

According to the Tomita-Takesaki Theorem, the existence of a cyclic separating vector implies that $\mathcal{M}$ is in symmetric form. According to [Bla06, Thm. III.4.5.6], any two realizations of $\mathcal{M}$ in symmetric form are unitarily equivalent.

Let $\mathfrak{S}_{n}(\mathcal{M})$ denote the set of normal states of the von Neumann algebra $\mathcal{M}$. By the Gelfand-Naimark-Segal construction, any state $\omega$ corresponds to a cyclic normal representation $\left(\pi_{\omega}, \mathcal{H}_{\omega}, \Omega_{\omega}\right)$ with $\omega(M)=\left\langle\Omega_{\omega}, \pi_{\omega}(M) \Omega_{\omega}\right\rangle$, which is uniquely determined up to unitary equivalence of cyclic representations. By construction $\Omega_{\omega}$ is cyclic, it leads to a faithful representation for which $\Omega_{\omega}$ is separating if and only if the state $\omega$ is faithful, i.e., $\omega\left(M^{*} M\right)>0$ for any non-zero $M \in \mathcal{M}$.

REMARK 4.5. (Existence of cyclic separating vectors) A von Neumann algebra $\mathcal{M}$ possesses a faithful normal state if and only if it is $\sigma$-finite (also called countably decomposable) in the sense that every family of mutually orthogonal projections in $\mathcal{M}$ is at most countable ([Bla06, Prop. III.4.5.3]). This is always the case if $\mathcal{M}$ can be realized on a separable Hilbert space, but not in general. Therefore one has to generalize the concept of a state to that of a normal weight. This is an additive positively homogeneous weakly lower semicontinuous functional $\omega: \mathcal{M}^{+} \rightarrow[0, \infty]$ on the positive cone $\mathcal{M}^{+}$of $\mathcal{M}$ that may also 
take the value $\infty$. A weight $\omega$ is called semifinite if the subset $\left\{M \in \mathcal{M}_{+}: \omega(M)<\infty\right\}$ generates $\mathcal{M}$ as a von Neumann algebra. Every von Neumann algebra has a faithful normal semifinite weight (cf. [Bla06. III.2.2.26]) and the GNS construction as well as Tomita-Takesaki theory extend naturally to normal semifinite weights. In particular, any such weight leads to a symmetric form realization of $\mathcal{M}$.

EXAmple 4.6. (a) Let $\mathcal{H}=L^{2}(X, \mathfrak{S}, \mu)$ for a $\sigma$-finite measure space $(X, \mathfrak{S}, \mu)$ and $\mathcal{M}=$ $L^{\infty}(X, \mathfrak{S}, \mu)$, acting on $\mathcal{H}$ by multiplication operators. Then the normal states of $\mathcal{M}$ are of the form $\omega_{h}(f)=\int_{X} f h d \mu$, where $0 \leq h$ satisfies $\int_{X} h d \mu=1$. Such a state is faithful if and only if $h \neq 0$ holds $\mu$-almost everywhere. Then $\Omega:=\sqrt{h} \in \mathcal{H}$ is a corresponding cyclic separating unit vector. From $S(f \Omega)=\bar{f} \Omega$, we obtain $S(f)=\bar{f}$, which is isometric and therefore $S=J$ and $\Delta=\mathbf{1}$.

(b) Let $\mathcal{H}=B_{2}(\mathcal{K})$ be the space of Hilbert-Schmidt operators on the complex separable Hilbert space $\mathcal{K}$ and consider the von Neumann algebra $\mathcal{M}=B(\mathcal{K})$ acting on $\mathcal{H}$ by left multiplications. Then $\mathcal{M}^{\prime} \cong B(\mathcal{K})^{\text {op }}$ acts by right multiplications. Normal states of $\mathcal{M}$ are of the form $\omega_{D}(A)=\operatorname{tr}(A D)$, where $0 \leq D$ satisfies $\operatorname{tr} D=1$. Such a state is faithful if and only if $\operatorname{ker} D=\{0\}$ (which requires $\mathcal{K}$ to be separable), and then $\Omega:=\sqrt{D} \in \mathcal{H}$ is a cyclic separating unit vector. Then $S(M \Omega)=M^{*} \Omega=(\Omega M)^{*}$ implies that

$$
J A=A^{*} \quad \text { and } \quad \Delta(A)=\Omega^{2} A \Omega^{-2}=D A D^{-1} \quad \text { for } \quad A \in B_{2}(\mathcal{K}) .
$$

(c) The prototypical pair $(\Delta, J)$ of a modular operator and a modular conjugation arises from the regular representation of a locally compact group $G$ on the Hilbert space $\mathcal{H}=L^{2}\left(G, \mu_{G}\right)$ with respect to a left Haar measure $\mu_{G}$. Here the modular operator is given by the multiplication

$$
\Delta f=\Delta_{G} \cdot f
$$

where $\Delta_{G}: G \rightarrow \mathbb{R}_{+}^{\times}$is the modular function of $G$ and the modular conjugation is given by

$$
(J f)(g)=\Delta_{G}(g)^{-\frac{1}{2}} \overline{f\left(g^{-1}\right)} .
$$

Accordingly, we have for $S=J \Delta^{1 / 2}$ :

$$
(S f)(g)=\Delta_{G}(g)^{-1} \overline{f\left(g^{-1}\right)}=f^{*}(g) .
$$

The corresponding von Neumann algebra is the algebra $\mathcal{M} \subseteq B\left(L^{2}\left(G, \mu_{G}\right)\right)$ generated by the left regular representation. If $M_{f} h=f * h$ is the left convolution with $f \in C_{c}(G)$, then the value of the corresponding normal weight $\omega$ on $\mathcal{M}$ is given by $\omega\left(M_{f}\right)=f(e)$, so that $\omega$ corresponds to evaluation in $e$, which is defined on a weakly dense subalgebra of $\mathcal{M}$.

4.2. Translating between standard subspaces and von Neumann algebras. We have already seen that cyclic separating vectors of a von Neumann algebra $\mathcal{M}$ lead to standard subspaces. In this subsection we explore some properties of this correspondence and describe how half-sided modular inclusions of standard subspaces translate into corresponding inclusions of von Neumann algebras. This correspondence shows that antiunitary representations of groups generated by modular one-parameter groups and conjugations from cyclic vectors of von Neumann algebras can already be studied in 
terms of standard subspaces and their inclusions, and all this can be encoded in antiunitary representations of pairs $\left(G, G_{1}\right)$, and homomorphisms $\mathbb{R}^{\times} \rightarrow G$, resp., Aff $(\mathbb{R}) \rightarrow G$ (Corollary 2.23 and Theorems 3.13, 3.22).

LEMmA 4.7. If $\mathcal{M} \subseteq B(\mathcal{H})$ is a von Neumann algebra and $\Omega \in \mathcal{H}$ a separating vector for $\mathcal{M}$, then we associate to every von Neumann subalgebra $\mathcal{N} \subseteq \mathcal{M}$ the closed real subspace $V_{\mathcal{N}}:=\overline{\mathcal{N}_{h} \Omega}$. This assignment is injective.

Note that the subspace $V_{\mathcal{N}}$ is standard if $\Omega$ is also cyclic for $\mathcal{N}$.

Proof. (cf. [Lo08, Prop. 3.24]) We have to show that $M \in \mathcal{M}_{h}$ and $M \Omega \in V_{\mathcal{N}}$ implies $M \in \mathcal{N}$. First we find a sequence $A_{n} \in \mathcal{N}$ such that $A_{n} \Omega \rightarrow M \Omega$. For any $B \in \mathcal{M}^{\prime}$, this leads to $A_{n} B \Omega=B A_{n} \Omega \rightarrow B M \Omega=M B \Omega$, so that $A_{n} \rightarrow M$ holds pointwise on the dense subspace $\mathcal{D}:=\mathcal{M}^{\prime} \Omega$. Since the hermitian operators $A_{n}$ and $M$ are bounded, $\mathcal{D}$ is a common core for all of them. With [RS73, Thm. VIII.25] it now follows that $A_{n} \rightarrow M$ holds in the strong resolvent sense, i.e., that $\left(i \mathbf{1}+A_{n}\right)^{-1} \rightarrow(i \mathbf{1}+M)^{-1}$ in the strong operator topology. This implies that $(i \mathbf{1}+M)^{-1} \in \mathcal{N}$, which entails $M \in \mathcal{N}$.

The concept of a half-sided modular inclusion was originally conceived on the level of von Neumann algebras with cyclic separating vectors, where it takes the following form ([Wi93, Wi97]).

DEFinition 4.8. Let $\Omega$ be a cyclic separating vector for the von Neumann algebra $\mathcal{M}$ and $\mathcal{N} \subseteq \mathcal{M}$ be a von Neumann subalgebra for which $\Omega$ is also cyclic. The triple $(\mathcal{M}, \mathcal{N}, \Omega)$ is called a \pm half-sided modular inclusion 4 if

$$
\Delta_{\mathcal{M}}^{-i t} \mathcal{N} \Delta_{\mathcal{M}}^{i t} \subseteq \mathcal{N} \quad \text { for } \quad \pm t \geq 0
$$

Note that $\Omega$ is also separating for $\mathcal{N}$ because $\mathcal{N} \subseteq \mathcal{M}$, so that we obtain two pairs of modular objects $\left(\Delta_{\mathcal{M}}, J_{\mathcal{M}}\right)$ and $\left(\Delta_{\mathcal{N}}, J_{\mathcal{N}}\right)$.

Lemma 4.9. Let $\mathcal{N} \subseteq \mathcal{M} \subseteq B(\mathcal{H})$ be von Neumann algebras with the common cyclic separating vector $\Omega \in \mathcal{H}$. Then $(\mathcal{M}, \mathcal{N}, \Omega)$ is a \pm half-sided modular inclusion if and only if the corresponding standard subspaces $V_{\mathcal{N}}:=\overline{\mathcal{N}_{h} \Omega} \subseteq V_{\mathcal{M}}:=\overline{\mathcal{M}_{h} \Omega}$ define a \pm half-sided modular inclusion.

Proof. Since $\overline{\Delta_{\mathcal{M}}^{-i t} \mathcal{N}_{h} \Delta_{\mathcal{M}}^{i t} \Omega}=\Delta_{\mathcal{M}}^{-i t} V_{\mathcal{N}}$, relation (16) implies

$$
\Delta_{\mathcal{M}}^{-i t} V_{\mathcal{N}} \subseteq V_{\mathcal{N}} \quad \text { for } \quad \pm t \geq 0 .
$$

If, conversely, the latter condition is satisfied, then $\Delta_{\mathcal{M}}^{-i t} \mathcal{N}_{h} \Delta_{\mathcal{M}}^{i t} \Omega \subseteq \Delta_{\mathcal{M}}^{-i t} V_{\mathcal{N}} \subseteq V_{\mathcal{N}}$, so that Lemma 4.7 implies (16).

The preceding lemma has a very interesting consequence because it translates directly between half-sided modular inclusions of von Neumann algebras and half-sided modular inclusions of the corresponding standard subspaces. It immediately implies that a triple $(\mathcal{M}, \mathcal{N}, \Omega)$ consisting of two von Neumann algebras $\mathcal{M}$ and $\mathcal{N}$ with a common cyclic separating vector $\Omega$ defines a modular intersection in the sense of Wi97] if and only if $V_{\mathcal{M}}$ and $V_{\mathcal{N}}$ have a modular intersection.

\footnotetext{
${ }^{4}$ Here we switched signs, compared to Bo97, Wi93, to make the concept compatible with the sign convention in the context of standard subspaces [Lo08].
} 
Clearly, every result on half-sided modular inclusions on standard subspaces, such as Borchers' Theorem 3.12 ([Bo00, Thms. II.5.2, VI.2.2]), Wiesbrock's Theorem 3.15 ([Wi93, AZ05]), and Theorem 3.19 (Wi98, Lemma 10]) yield corresponding results on half-sided modular inclusions of von Neumann algebras which preceded the corresponding results on standard subspaces.

It is remarkable that this transfer also works in the other direction: every result on half-sided modular inclusions of von Neumann algebras can be used to obtain a corresponding result on standard subspaces. For this transfer one can use the second quantization procedure described in some detail in Section 6 below. It associates to every standard subspace $V \subseteq \mathcal{H}$ a von Neumann algebra $\mathcal{R}(V) \subseteq B\left(\mathcal{F}_{+}(\mathcal{H})\right)$ on the bosonic Fock space $\mathcal{F}_{+}(\mathcal{H})$ for which the vacuum $\Omega$ is a cyclic separating vector and for which the modular objects are obtained by second quantization. Here we consider the antiunitary representation

$$
\Gamma: \operatorname{AU}(\mathcal{H}) \rightarrow \operatorname{AU}\left(\mathcal{F}_{+}(\mathcal{H})\right), \quad \Gamma(U)\left(v_{1} \vee \cdots \vee v_{n}\right):=U v_{1} \vee \cdots \vee U v_{n}
$$

obtained by second quantization. If $\gamma_{V}: \mathbb{R}^{\times} \rightarrow \mathrm{AU}(\mathcal{H})$ is the antiunitary representation associated to $V$, then $\widetilde{\gamma}_{V}:=\Gamma \circ \gamma_{V}$ is the corresponding antiunitary representation on the Fock space $\mathcal{F}_{+}(\mathcal{H})$ (cf. Proposition 3.2).

If $\Delta_{V}^{-i t / 2 \pi} H=\gamma_{V}\left(e^{t}\right) H \subseteq H$ holds for $t \geq 0$, then

$$
\mathcal{R}\left(\gamma_{V}\left(e^{t}\right) H\right)=\widetilde{\gamma}_{V}\left(e^{t}\right) \mathcal{R}(H) \widetilde{\gamma}_{V}\left(e^{-t}\right) \subseteq \mathcal{R}(H)
$$

implies that $(\mathcal{R}(V), \mathcal{R}(H), \Omega)$ is a \pm half-sided modular inclusion whenever $H \subseteq V$ is.

If, conversely, $(\mathcal{R}(V), \mathcal{R}(H), \Omega)$ is a \pm half-sided modular inclusion, then

$$
\mathcal{R}\left(\gamma_{V}\left(e^{t}\right) H\right)=\widetilde{\gamma}_{V}\left(e^{t}\right) \mathcal{R}(H) \widetilde{\gamma}_{V}\left(e^{-t}\right) \subseteq \mathcal{R}(H)
$$

implies that $\gamma_{V}\left(e^{t}\right) H \subseteq H$ by Theorem 6.4(i). Therefore $H \subseteq V$ is a half-sided modular inclusion of the same type. As the subgroup of $\mathrm{AU}\left(\mathcal{F}_{+}(\mathcal{H})\right)$ generated by the corresponding one-parameter groups $\widetilde{\gamma}_{V}\left(\mathbb{R}^{\times}\right)$is contained in the subgroup $\Gamma(\mathrm{AU}(\mathcal{H}))$ which is the range of the second quantization homomorphism $\Gamma: \operatorname{AU}(\mathcal{H}) \rightarrow \operatorname{AU}\left(\mathcal{F}_{+}(\mathcal{H})\right.$ ), anything that we can say about subgroups generated by these groups and conditions relating to modular objects can be translated into a corresponding result on standard subspaces and the antiunitary one-parameter groups $\gamma_{V}$ on $\mathcal{H}$.

According to this principle, any result on half-sided modular inclusions of von Neumann algebras has a "one-particle version" concerning standard subspaces and vice versa (cf. $\$ \$ 3.4$ 3.5). The advantage of the one-particle version is that it has a simpler formulation and that standard subspaces are completely encoded in the antiunitary representations $\gamma_{V}$ of $\mathbb{R}^{\times}$, hence in an antiunitary representation of a group $\left(G, G_{1}\right)$ generated by the image of homomorphism $\left(\mathbb{R}^{\times}, \mathbb{R}_{+}^{\times}\right) \rightarrow\left(G, G_{1}\right)$. Therefore one can hope that any results on standard subspaces, half-sided modular inclusions and the corresponding groups can be expressed in terms of antiunitary representations of suitable involutive pairs of Lie groups $\left(G, G_{1}\right)$. This was one of the key motivations for us to write this note.

REMARK 4.10. (a) A typical result of this type is Wiesbrock's Theorem on half-sided modular inclusions (cf. Theorem 3.15 and Wi93, Wi97, AZ05]). On the level of modular inclusions of von Neumann algebras $(\mathcal{M}, \mathcal{N}, \Omega)$, Wiesbrock provides the additional infor- 
mation that, if $\mathcal{M}$ is a factor, then it is of type $\mathrm{III}_{1}$ (see Wi93, Thm. 12] which uses Lo82]). It would be interesting to see if and how this can be formulated and derived on the level of standard subspaces and antiunitary representations. The discussion of modular nuclearity in [Lo08, §6.3] may indicate a possible way how this can be done.

(b) In [GLW98, Thm. 4.11] (see also Wi93c, Lemmas 3,4ff] and [Wi93b, Thm. 2]), similar structures related to multiple modular inclusions are studied, namely quadruples $\left(\mathcal{M}_{0}, \mathcal{M}_{1}, \mathcal{M}_{2}, \Omega\right)$, where the $\mathcal{M}_{j}$ are von Neumann algebras with the common separating cyclic vector $\Omega$ such that the $\mathcal{M}_{j}$ commute pairwise and, in cyclic order, $\mathcal{M}_{j} \subseteq \mathcal{M}_{j+1}^{\prime}$ is a half sided modular inclusion. From this structure, which arises from partitions of $\mathbb{S}^{1}$ into three intervals, one derives antiunitary positive energy representations of $\mathrm{PGL}_{2}(\mathbb{R})$ as in Theorem 3.19 (GLW98, Thm. 1.2]).

(c) In Wi93b] it is shown that the von Neumann version of Theorem 3.19(b) characterizes conformal quantum fields on the circle in terms of modular data associated to three intervals.

(d) In [KW01] configurations of 6 von Neumann algebras $\left(\mathcal{M}_{i j}\right)_{1 \leq i<j \leq 4}$ are used to generate unitary representations of the group $\mathrm{SO}_{1,3}(\mathbb{R})^{\uparrow}$ and further of the connected Poincaré group $P(4)_{+}^{\uparrow}$.

4.3. Borchers triples. In this subsection we briefly discuss generalization of Borchers pairs to higher dimensional situations, where the semigroup $\mathbb{R}_{+}$acting on a standard subspace is replaced by a wedge $W$ in Minkowski space or by the subsemigroup of $P(d)_{+}$ mapping such a wedge into itself.

Definition 4.11. In $d$-dimensional Minkowski space $\mathbb{R}^{1, d-1}$, we consider the right wedge

$$
W_{R}:=\left\{x=\left(x_{0}, \ldots, x_{d-1}\right) \in \mathbb{R}^{d}: x_{1}>\left|x_{0}\right|\right\} .
$$

To fix notation for the following, we write $W_{R}=W_{R}^{2} \oplus E_{R}$, where

$$
E_{R}=\left\{\left(x_{0}, \mathbf{x}\right): x_{0}=x_{1}=0\right\} \cong \mathbb{R}^{d-2}
$$

is the edge of the wedge and $W_{R}^{2}$ is the standard right wedge in $\mathbb{R}^{2}$.

A subset of the form $W=g W_{R}, g \in P(d)$, is called a wedge. We write $\mathcal{W}$ for the set of wedges in $\mathbb{R}^{1, d-1}$.

The following lemma contains some details on $\mathcal{W}$ as an ordered homogeneous space. For item (iii), we recall the generator $b_{0}$ of the Lorentz boost from Example 2.27 (see also [BGL02, §2]).

LEMMA 4.12. The wedge space $\mathcal{W}$ has the following properties:

(i) The stabilizer $P(d)_{W_{R}}=\left\{g \in P(d): g W_{R}=W_{R}\right\}$ of the standard right wedge has the form

$$
P(d)_{W_{R}} \cong E(d-2) \times \mathrm{O}_{1,1}(\mathbb{R})_{W_{R}^{2}},
$$

where $E(d-2)$ denotes the euclidean group on $E_{R} \cong \mathbb{R}^{d-2}$.

(ii) $r_{W}:=g R_{01} g^{-1}$ for $W=g W$ and $R_{01}=\operatorname{diag}(-1,-1,1, \ldots, 1)$ yields a consistent definition of wedge reflections $\left(r_{W}\right)_{W \in \mathcal{W}}$.

(iii) The subgroup $P(d)^{\uparrow}$ acts transitively on $\mathcal{W}$, and the following are equivalent for $g \in P(d)^{\uparrow}:$ 
(a) $g W_{R}=W_{R}$.

(b) $\operatorname{Ad}_{g} b_{0}=b_{0}$ and $g$ commutes with the wedge reflection $r_{W_{R}}=R_{01}$.

(c) $\operatorname{Ad}_{g} b_{0}=b_{0}$.

The set of all elements satisfying these conditions is

$$
P(d)_{W_{R}}^{\uparrow} \cong E(d-2) \times \mathrm{SO}_{1,1}(\mathbb{R})^{\uparrow} .
$$

In particular, the subgroup $\mathrm{SO}_{1,1}(\mathbb{R})^{\uparrow}$ is central in $P(d)_{W_{R}}^{\uparrow}$. For $d>2$, even the identity component acts transitively on $\mathcal{W}$ with stabilizer

$$
P(d)_{+, W_{R}}^{\uparrow} \cong E(d-2)_{+} \times \mathrm{SO}_{1,1}(\mathbb{R})^{\uparrow} .
$$

(iv) For $\gamma_{W_{R}}: \mathbb{R}^{\times} \rightarrow P(d)_{+}$, defined by $\gamma_{W_{R}}\left(e^{t}\right):=e^{t b_{0}}$ and $\gamma_{W_{R}}(-1):=r_{W_{R}}$, we have a bijection

$$
\mathcal{W} \rightarrow C_{\gamma_{W_{R}}}, \quad g W_{R} \mapsto \gamma_{W}:=\gamma_{W_{R}}^{g} \quad \text { for } \quad g \in P(d)_{+}^{\uparrow}, \quad \gamma_{W_{R}}^{g}(t)=g \gamma_{W_{R}}(t) g^{-1}
$$

and the map

$$
\mathcal{W} \rightarrow C_{r_{W_{R}}}=\left\{r_{W}: W \in \mathcal{W}\right\}, \quad W \mapsto r_{W}
$$

corresponding to evaluation in -1 is a two-fold covering map.

(v) We have a bijection $\mathcal{W} \rightarrow \operatorname{Ad}\left(P(d)^{\uparrow}\right) b_{0}, g W_{R} \mapsto \operatorname{Ad}(g) b_{0}$ of $\mathcal{W}$ onto an adjoint orbit of $P(d)^{\uparrow}$.

(vi) The stabilizer $P(d)_{W_{R}}$ is open in the centralizer of $r_{W_{R}}$ in $P(d)$. In particular $\left(P(d), P(d)_{W_{R}}\right)$ is a symmetric pair and $\mathcal{W}$ is a symmetric space.

(vii) The semigroup $S_{W_{R}}:=\left\{g \in P(d): g W_{R} \subseteq W_{R}\right\}$ is given by $\overline{W_{R}} \rtimes \mathrm{O}_{1, d-1}(\mathbb{R})_{W_{R}}$.

Proof. (i) The stabilizer group contains the translation group corresponding to the edge $E_{R}$ and $g W_{R}=W_{R}$ implies $g(0) \in E_{R}$, so that

$$
P(d)_{W_{R}} \cong E_{R} \rtimes \mathrm{O}_{1, d-1}(\mathbb{R})_{W_{R}} .
$$

Further,

$$
\mathrm{O}_{1, d-1}(\mathbb{R})_{W_{R}}=\mathrm{O}_{d-2}(\mathbb{R}) \times \mathrm{O}_{1,1}(\mathbb{R})_{W_{R}^{2}}=\mathrm{O}_{d-2}(\mathbb{R}) \times\left(\mathrm{SO}_{1,1}(\mathbb{R})^{\uparrow}\left\{\mathbf{1}, R_{1}\right\}\right),
$$

where $R_{1}=\operatorname{diag}(1,-1,1, \ldots, 1)$. We thus obtain (i).

(ii) follows from the fact that the wedge reflection $R_{01}$ commutes with $P(d)_{W_{R}}$.

(iii) That $P(d)^{\uparrow}$ acts transitively follows from the fact that the stabilizer $P(d)_{W_{R}}$ contains the reflection $R_{1}$ satisfying $R_{1} V_{+}=-V_{+}$. If $d>2$, then the stabilizer $P(d)_{W_{R}}$ intersects all four connected components of $P(d)$, so that even $P(d)_{+}^{\uparrow}$ acts transitively. For $d=2$ we obtain two orbits because $\pm W_{R}$ lie in different orbits of $P(2)_{+}^{\uparrow}$.

It remains to verify the equivalence of (a), (b) and (c). From (18) we derive that (a) implies (b) and hence (c). That $g=(b, a)$ commutes with $R_{01}$ is equivalent to $b \in E_{R}$ and $a=a_{1} \oplus a_{2}$ with $a_{1}$ acting on the first two coordinates and $a_{2}$ on $E_{R}$. That, in addition, $g$ commutes with $b_{0}$ restricts $a_{1} \in \mathrm{O}_{1,1}(\mathbb{R})^{\uparrow}$ to an element of $\mathrm{SO}_{1,1}(\mathbb{R})^{\uparrow}$. Finally, we observe that, if $g$ commutes with $b_{0}$, then the eigenspace decomposition of $\operatorname{ad} b_{0}$ on $\mathfrak{p}(d)$ implies that $g=(b, a)$ with $b \in E_{R}$ and $a=a_{1} \oplus a_{2}$ with $a_{1} \in \mathrm{SO}_{1,1}(\mathbb{R})^{\uparrow}$.

(iv) The first part follows from the equivalence of (a) and (b) in (iii). For the second part, we observe with (iii) above that the stabilizer of $W_{R}$ is a subgroup of index 2 in the centralizer of $r_{W_{R}}$. 
(v) follows from the equivalence of (a) and (c) in (iii).

(vi) The centralizer of $r_{W_{R}}$ in $P(d)$ is the subgroup $E(d-2) \times \mathrm{O}_{1,1}(\mathbb{R})$ in which the stabilizer group $P(d)_{W_{R}}$ is open. This means that $\left(P(d), P(d)_{W_{R}}\right)$ is a symmetric pair.

(vii) For a closed convex subset $C \subseteq \mathbb{R}^{d}$, its recession cone

$$
\lim (C):=\left\{x \in \mathbb{R}^{d}: x+C \subseteq C\right\}=\left\{x \in \mathbb{R}^{d}:(\exists c \in C) c+\mathbb{R}_{+} x \subseteq C\right\}
$$

is a closed convex cone ([Ne0, Prop. V.1.6]), and each affine map $g=(b, a) \in$ $\mathbb{R}^{d} \rtimes \mathrm{GL}_{d}(\mathbb{R}) \cong \operatorname{Aff}\left(\mathbb{R}^{d}\right)$ satisfies

$$
\lim (g C)=\lim (a C)=a \lim (C) .
$$

If $g=(b, a) \in S_{W_{R}}$, then $g$ maps $\overline{W_{R}}$ into itself, so that $b=g(0) \in \overline{W_{R}}$. Further (20) implies that $\overline{W_{R}} \supseteq \lim \left(g W_{R}\right)=a \overline{W_{R}}$, and hence $a W_{R} \subseteq W_{R}$. It follows that $a E_{R} \subseteq E_{R}$, so that $a E_{R}=E_{R}$ as $a$ is injective and $\operatorname{dim} E_{R}<\infty$. This in turn implies that $a$ commutes with $r_{W_{R}}$, so that $a=a_{1} \oplus a_{2}$ as above, where $a_{2} \in \mathrm{O}\left(E_{R}\right)$ and $a_{1} W_{R}^{2} \subseteq W_{R}^{2}$. As $a_{1} W_{R}^{2}$ is a quarter plane bounded by light rays, we get $a_{1} W_{R}^{2}=W_{R}^{2}$, and finally $a W_{R}=W_{R}$.

Definition 4.13. ([Le15, Def. 2.7]) A d-dimensional standard pair $(V, U)$ with translation symmetry relative to $W \in \mathcal{W}$ consists of a standard subspace $V \subseteq \mathcal{H}$ and a strongly continuous unitary positive energy representation $U$ of the translation group $\mathbb{R}^{d}$ (cf. Definition 2.35) such that $U_{x} V \subseteq V$ whenever $x+W \subseteq W$.

Here is the corresponding concept for von Neumann algebras:

Definition 4.14. ([BLS11, §4]) A (causal) Borchers triple $(\mathcal{M}, U, \Omega)$ relative to the wedge $W \subseteq \mathbb{R}^{d}$ consists of

(B1) a von Neumann algebra $\mathcal{M} \subseteq B(\mathcal{H})$,

(B2) a positive energy representation $(U, \mathcal{H})$ of the translation group $\mathbb{R}^{d}$ such that $U_{x} \mathcal{M} U_{x}^{*} \subseteq \mathcal{M}$ if $x+W \subseteq W$, and

(B3) a $U$-invariant unit vector $\Omega \in \operatorname{cs}(\mathcal{M})$.

REmark 4.15. Let $\mathcal{M} \subseteq B(\mathcal{H})$ be a von Neumann algebra, $U: \mathbb{R}^{d} \rightarrow \mathrm{U}(\mathcal{H})$ be a continuous unitary representation and $\Omega \in \mathcal{H}^{U} \cap \operatorname{cs}(\mathcal{M})$. We consider the corresponding standard subspace $V:=\overline{\mathcal{M}}_{h} \Omega$. Then $U_{x} V \subseteq V$ is equivalent to $U_{x} \mathcal{M} U_{x}^{*} \subseteq \mathcal{M}$ by Lemma 4.7. Therefore $(\mathcal{M}, U, \Omega)$ is a Borchers triple with respect to $W$ if and only if $(V, U)$ is a standard pair with respect to $W$.

The following theorem can be obtained by translating Bo92 from the context of Borchers triples to standard pairs by arguing as in $\$ 4.2$. We give a direct proof based on our Theorem 3.12

THEOREM 4.16 (Borchers' standard pair Theorem). Let $(V, U)$ be a d-dimensional standard pair with translation symmetry relative to $W_{R}$ and $\gamma_{W_{R}}: \mathbb{R}^{\times} \rightarrow \mathrm{SO}_{1, d-1}(\mathbb{R})$ be the corresponding homomorphism with $\gamma_{W_{R}}\left(e^{t}\right)=e^{t b_{0}}$ and $\gamma_{W_{R}}(-1)=r_{W_{R}}=R_{01}$ (Lemma $4.12(\mathrm{iv}))$. Then the antiunitary representation $\left(U^{V}, \mathcal{H}\right)$ of $\mathbb{R}^{\times}$corresponding to $V$ satisfies

$$
U_{t}^{V} U_{x} U_{t^{-1}}^{V}=U_{\gamma_{W_{R}}(t) x} \quad \text { for } \quad x \in \mathbb{R}^{d}, t \in \mathbb{R}^{\times},
$$

so that we obtain an antiunitary representation of $\mathbb{R}^{d} \rtimes \mathrm{SO}_{1,1}(\mathbb{R})$ by $\left(b, \gamma_{W_{R}}(t)\right) \mapsto U_{b} U_{t}^{V}$. 
Conversely, every antiunitary positive energy representation $(U, \mathcal{H})$ of $\mathbb{R}^{d} \rtimes \mathrm{SO}_{1,1}(\mathbb{R})$ defines a standard pair $\left(V_{\gamma_{W_{R}}},\left.U\right|_{\mathbb{R}^{d}}\right)$.

Proof. First we write $\mathbb{R}^{d}=\mathbb{R}^{1,1} \oplus \mathbb{R}^{d-2}$, so that $W_{R}=W_{R}^{2} \oplus \mathbb{R}^{d-2}$, where $W_{R}^{2} \subseteq \mathbb{R}^{1,1}$ is the standard right wedge. For the light-like vectors $\ell_{ \pm}:=(1, \pm 1,0, \ldots, 0)$ we then have $W_{R}^{2}=\mathbb{R}_{+}^{\times} \ell_{+}-\mathbb{R}_{+}^{\times} \ell_{-}$. By assumption, $U_{t \ell_{ \pm}}=e^{i t P_{ \pm}}$with $P_{ \pm} \geq 0$. The strong continuity of $U$ implies

$$
U_{x} V \subseteq V \quad \text { for all } \quad x \in \bar{W}=\left([0, \infty) \ell_{+}-[0, \infty) \ell_{-}\right) \oplus \mathbb{R}^{d-2} .
$$

Now Theorem 3.12 yields

$$
U_{e^{t}}^{V} U_{s \ell_{ \pm}} U_{e^{-t}}^{V}=U_{e^{ \pm t}} s \ell_{ \pm} \quad \text { for } \quad t, s \in \mathbb{R} .
$$

Further, $U_{x} V=V$ for $x=\left(0,0, x_{2}, \ldots, x_{d-1}\right)$ implies that $U_{x}$ commutes with $\Delta_{V}$. Combing all this, the first assertion follows.

For the converse, let $(U, \mathcal{H})$ be an antiunitary positive energy representation of the group $\mathbb{R}^{d} \rtimes \mathrm{SO}_{1,1}(\mathbb{R})$ and $V=V_{\gamma_{W_{R}}}$ be the standard subspace corresponding to $\gamma^{V}:=$ $U \circ \gamma_{W_{R}}$ by (Proposition 3.2). Since $\gamma_{W_{R}}$ commutes with $E_{R}$, the subgroup $U_{E_{R}}$ commutes with $\gamma^{U}$ and leaves $V$ invariant. That $U_{x} V \subseteq V$ for $x \in W_{R}^{2}$ follows from the positive energy condition and Theorem $\underline{3.13}$ (ii).

Here is a variant of this concept where the translation group is replaced by the Poincaré group:

Definition 4.17. A $d$-dimensional standard pair $(V, U)$ with Poincaré symmetry relative to $W_{R} \in \mathcal{W}$ consists of a standard subspace $V \subseteq \mathcal{H}$ and a strongly continuous unitary positive energy representation $U$ of the connected Poincaré group $P(d)_{+}^{\uparrow}$, such that

(i) $U_{g} V \subseteq V$ for all $g \in P(d)_{+}^{\uparrow}$ with $g W_{R} \subseteq W_{R}$ and

(ii) $U_{g} V \subseteq V^{\prime}$ for all $g \in P(d)_{+}^{\uparrow}$ with $g W_{R} \subseteq-W_{R}$.

LEMma 4.18. Let $(U, \mathcal{H})$ be an antiunitary positive energy representation of $P(d)_{+}$for $d \geq 3$ and $V \subseteq \mathcal{H}$ be the standard subspace corresponding to the canonical homomorphism $\gamma_{W_{R}}: \mathbb{R}^{\times} \rightarrow P(d)_{+}$. Then $(V, U)$ is a standard pair with Poincaré symmetry.

Proof. If $g W_{R}=W_{R}$, then $g \in P(d)_{+}$commutes with $\gamma_{W_{R}}$ by Lemma 4.12(iii), so that $U_{g} V=V$. Further $U_{x} V \subseteq V$ for $x \in W_{R}$ (and hence also for $x \in \overline{W_{R}}$ by continuity) follows from the second part of Theorem 4.16. In view of Lemma 4.12)(vii), this implies that $U_{g} V \subseteq V$ if $g W_{R} \subseteq W_{R}$.

If $g W_{R} \subseteq-W_{R}$, then the element $r:=R_{12}=\operatorname{diag}(1,-1,-1,1, \ldots, 1) \in P(d)_{+}^{\uparrow}$ satisfies $r g W_{R} \subseteq r\left(-W_{R}\right)=W_{R}$, so that the above argument leads to $U_{g} V=U_{r} U_{r g} V \subseteq$ $U_{r} V$. Now $\gamma_{W_{R}}^{r}=\gamma_{W_{R}}^{\vee}$ yields $U_{r} V=V^{\prime}=V_{\gamma_{W_{R}}^{\vee}}$, so that $U_{g} V \subseteq V^{\prime}$.

REMARK 4.19. (a) In [BLS11, standard pairs with Poincaré symmetry are used to obtain Borchers triples by second quantization (cf. Section 6). Composing with a deformation process due to Rieffel, this construction yields non-free quantum fields in arbitrary large dimensions.

(b) The main point of the notion of a Borchers triple is that they can be used to construct a representation of the Poincaré group $P(d)_{+}^{\uparrow}$ by generating it with modular 
one-parameter groups of a finite set of von Neumann algebras with a common cyclic separating vector (Bo96, Wi93c, Wi98, SW00, KW01]).

(c) For $d=1$, we think of $\mathbb{R}=\mathbb{R}^{d}$ as the underlying space as a light ray in Minkowski space, so that the Poincaré group is replaced by the affine group Aff(R). In this context unitary "endomorphisms" of irreducible one-dimensional standard pairs (Definition 4.13) are unitary operators $W \in \mathrm{U}(\mathcal{H})$ commuting with the one-parameter group $U$ satisfying $W V \subseteq V$. If $P$ is the momentum operator determined by $U_{t}=e^{i t P}$, then these are precisely the operators of the form $W=\varphi(P)$, where $\varphi$ is a symmetric inner function on the upper half plane $\mathbb{C}_{+} \subseteq \mathbb{C}$ and $P$ is the momentum operator. A symmetric inner function is a bounded holomorphic function on $\mathbb{C}_{+}$satisfying

$$
\varphi(p)^{-1}=\overline{\varphi(p)}=\varphi(-p) \quad \text { for almost all } \quad p \in \mathbb{R}
$$

([LW11, Cor. 2.4]; see also Example 3.16). That these functions can be used to construct Borchers triples was shown by Tanimoto in [Ta12].

Much more could be said about the structured related to standard subspaces, halfsided modular inclusions, modular intersections etc.. For more details and an in depth study of these concepts, we refer to Bo97, and Wiesbrock's work Wi93c, Wi97b, Wi98.

4.4. Modular geometry. In this subsection we discuss some of the geometric structures arising from a single von Neumann algebra $\mathcal{M} \subseteq B(\mathcal{H})$ which has cyclic separating vectors. Any such vector $\xi$ leads to a standard subspace $V_{\xi}=\overline{\mathcal{M}_{h} \xi}$ and corresponding modular objects $\left(\Delta_{\xi}, J_{\xi}\right)$ (Theorem 4.2). Fixing a cyclic separating vector $\Omega$, the associated natural cone provides a means to analyze the orbits of the group generated by $\mathrm{U}(\mathcal{M}), \mathrm{U}\left(\mathcal{M}^{\prime}\right)$ and the modular conjugations on the data.

Definition 4.20. We consider a von Neumann algebra $\mathcal{M} \subseteq B(\mathcal{H})$ for which the set $\operatorname{cs}(\mathcal{M})$ of cyclic and separating unit vectors is non-empty. We fix an element $\Omega \in \operatorname{cs}(\mathcal{M})$ and the corresponding modular objects $(\Delta, J)$ (Theorem 4.2). We recall the natural positive cone

$$
\mathcal{P}:=\overline{\{A j(A) \Omega: A \in \mathcal{M}\}}, \quad \text { where } \quad j(A):=J A J
$$

([BR87, Def. 2.5.25]) and write

$$
\operatorname{cs}(\mathcal{M})_{+}:=\mathcal{P} \cap \operatorname{cs}(\mathcal{M})
$$

for the set of cyclic separating unit vectors in $\mathcal{P}$. We further write

$$
\operatorname{mc}(\mathcal{M}):=\left\{J_{\xi}: \xi \in \operatorname{cs}(\mathcal{M})\right\}
$$

for the corresponding set of modular conjugations. We further consider the set

$$
\operatorname{ms}(\mathcal{M})=\left\{V_{\xi}=\overline{\mathcal{M}_{h} \xi}: \xi \in \operatorname{cs}(\mathcal{M})\right\} \subseteq \operatorname{Stand}(\mathcal{H})
$$

of modular standard subspaces for $\mathcal{M}$ and note that $\Delta_{V_{\xi}}=\Delta_{\xi}$ and $J_{V_{\xi}}=J_{\xi}$.

We write $\mathcal{Z}:=\mathcal{M} \cap \mathcal{M}^{\prime}$ for the center of $\mathcal{M}$.

Proposition 4.21. The following assertions hold:

(i) (Polar decomposition of $\operatorname{cs}(\mathcal{M}))$ The map $\mathrm{U}\left(\mathcal{M}^{\prime}\right) \times \operatorname{cs}(\mathcal{M})_{+} \rightarrow \operatorname{cs}(\mathcal{M}),(U, \xi) \mapsto U \xi$ is a bijection. 
(ii) The unitary groups $\mathrm{U}(\mathcal{M})$ and $\mathrm{U}\left(\mathcal{M}^{\prime}\right)$ both act transitively on $\operatorname{mc}(\mathcal{M})$ by conjugation. For $J \in \operatorname{mc}(\mathcal{M})$, the stabilizer in both groups is the discrete central subgroup

$$
\mathrm{U}\left(\mathcal{M}^{\prime}\right)_{J}=\mathrm{U}(\mathcal{M})_{J}=\operatorname{Inv}(\mathrm{U}(\mathcal{Z}))=\left\{z \in \mathrm{U}(\mathcal{Z}): z^{2}=\mathbf{1}\right\}
$$

of central unitary involutions. The orbit map $\sigma: \mathrm{U}(\mathcal{M}) \rightarrow \operatorname{mc}(\mathcal{M}), \sigma(U):=U U^{-1}$ is a covering morphism of Banach-Lie groups if we identify $\operatorname{mc}(\mathcal{M})$ with the quotient $\mathrm{U}(\mathcal{M}) / \operatorname{ker} \sigma$.

(iii) $\operatorname{grp}(\operatorname{mc}(\mathcal{M}))=\operatorname{Comm}(\mathrm{U}(\mathcal{M})) \operatorname{mc}(\mathcal{M})\{\mathbf{1}, J\}$, where $\operatorname{Comm}(\mathrm{U}(\mathcal{M}))$ is the commutator subgroup of $\mathrm{U}(\mathcal{M})$.

(iv) $\operatorname{cs}(\mathcal{M})_{J}:=\left\{\xi \in \operatorname{cs}(\mathcal{M}): J_{\xi}=J\right\}=\operatorname{Inv}(\mathrm{U}(\mathcal{Z})) \operatorname{cs}(\mathcal{M})_{+}$.

(v) The stabilizer of $J$ in the group $\mathrm{U}(\mathcal{M}) U\left(\mathcal{M}^{\prime}\right)$ is $\{U j(U): U \in \mathrm{U}(\mathcal{M})\} \operatorname{Inv}(\mathrm{U}(\mathcal{Z}))$.

(vi) For $\xi \in \operatorname{cs}(\mathcal{M})$, we have $V_{\xi}=V_{\Omega}$ if and only if there exists a selfadjoint operator $Z$ affiliated with $\mathcal{Z}$, i.e., commuting with $\mathrm{U}(\mathcal{M}) \mathrm{U}\left(\mathcal{M}^{\prime}\right)$, such that $\xi=Z \Omega$.

Proof. (i) For any $\xi \in \operatorname{cs}(\mathcal{M})$, there exists a unit vector $\widetilde{\xi} \in \mathcal{P}$ defining the same state of $\mathcal{M}$ ([BR87, Thm. 2.5.31]). By the GNS Theorem, there exists a $U \in \mathrm{U}\left(\mathcal{M}^{\prime}\right)$ with $\xi=U \widetilde{\xi}$. Since the elements of $\operatorname{cs}(\mathcal{M})_{+}$are also separating for $\mathcal{M}^{\prime}$, their stabilizer in $\mathrm{U}\left(\mathcal{M}^{\prime}\right)$ is trivial.

To verify injectivity, it remains to see that every $\mathrm{U}\left(\mathcal{M}^{\prime}\right)$-orbit in $\operatorname{cs}(\mathcal{M})$ meets $\operatorname{cs}(\mathcal{M})_{+}$ exactly once. Let $U \in \mathrm{U}\left(\mathcal{M}^{\prime}\right)$ and $\xi \in \operatorname{cs}(\mathcal{M})_{+}$be such that $U \xi \in \mathcal{P}$. As $J_{\xi}=J$ for every $\xi \in \mathcal{P}$ by [BR87, Prop. 2.5.30], we obtain $U J U^{-1}=J_{U \xi}=J$. Then $j(U)=U$ leads to $U \in \mathcal{M} \cap \mathcal{M}^{\prime}$ and hence to $U=j(U)=U^{-1}$ (Theorem 4.2(c)), so that $U^{2}=1$. Then $\mathcal{M}_{ \pm 1}:=\{M \in \mathcal{M}: U M= \pm M\}$ are ideals of $\mathcal{M}$ and $\mathcal{M} \cong \mathcal{M}_{+} \oplus \mathcal{M}_{-}$is a direct sum of von Neumann algebras. Now $\xi=\xi_{+} \oplus \xi_{-}$decomposes accordingly with $\xi_{ \pm} \in \operatorname{cs}\left(\mathcal{M}_{ \pm}\right)$. As $U \xi=\xi_{+}-\xi_{-}$and $\mathcal{P}=\mathcal{P}_{+} \oplus \mathcal{P}_{-}$, it follows that $\xi_{-} \in \mathcal{P}_{-} \cap-\mathcal{P}_{-}=\{0\}([$ BR87, Prop. 2.5.28]) and thus $\mathcal{M}_{-}=\{0\}$ and $U=\mathbf{1}$.

(ii) If $\Omega_{1}, \Omega_{2} \in \operatorname{cs}(\mathcal{M})$, then [BR87, Lemma 2.5.35] implies the existence of a unitary element $U \in \mathrm{U}\left(\mathcal{M}^{\prime}\right)$ with $U J_{\Omega_{1}} U^{-1}=J_{\Omega_{2}}$. Exchanging the roles of $\mathcal{M}$ and $\mathcal{M}^{\prime}$, it also follows that $\mathrm{U}(\mathcal{M})$ acts transitively on $\operatorname{mc}(\mathcal{M})$.

For $J \in \operatorname{mc}(\mathcal{M})$ we have $J \mathcal{M} J=\mathcal{M}^{\prime}$, so that, for $U \in \mathrm{U}(\mathcal{M})$, the relation $U J U^{-1}=J$ implies $U=J U J \in \mathcal{Z}$. As in (i), this leads to $J U J=U^{*}=U^{-1}$, so that $U^{2}=\mathbf{1}$. Conversely, any involution in $\mathrm{U}(\mathcal{Z})$ stabilizes $J$.

Clearly, $\sigma$ is a surjective equivariant map whose kernel is discrete in the norm topology. As the stabilizer subgroup of $J$ in $\mathrm{U}(\mathcal{M})$ is discrete and central, the quotient $\mathrm{U}(\mathcal{M}) / \operatorname{ker} \sigma$ carries a natural Banach-Lie group structure for which $\sigma$ becomes a covering homomorphism.

(iii) We consider the group $G:=\mathrm{U}\left(\mathcal{M}^{\prime}\right)$ and the representation of $(G \times G) \rtimes\{\mathbf{1}, \tau\}$ on $\mathcal{H}$ given by $U\left(g, h, \tau^{\varepsilon}\right)=g J h J J^{\varepsilon}$. Then Proposition 4.21 shows that

$$
U\left(C_{(e, e, \tau)}\right)=\left\{g J g^{-1} J J: g \in \mathrm{U}\left(\mathcal{M}^{\prime}\right)\right\}=\left\{g J g^{-1}: g \in \mathrm{U}\left(\mathcal{M}^{\prime}\right)\right\}=\operatorname{mc}(\mathcal{M}) .
$$

Now the assertion follows from Lemma A.3.

(iv) We have already seen in (i) that $J_{\xi}=J$ for every $\xi \in \operatorname{cs}(\mathcal{M})_{+}$. If $\xi=U \widetilde{\xi}$ for some $U \in \mathrm{U}\left(\mathcal{M}^{\prime}\right)$ and $\widetilde{\xi} \in \operatorname{cs}(\mathcal{M})_{+}$as in (i), then $J_{\xi}=U J U^{-1}$ equals $J$ if and only if $U \in \mathcal{M} \cap \mathcal{M}^{\prime}$ is an involution. This proves (iv). 
(v) Since $J$ commutes with each operator of the form $J U J U=j(U) U=U j(U)$, the stabilizer contains all these elements and also $\operatorname{Inv}(\mathcal{Z})$, as we have already seen above. If, conversely, $U \in \mathrm{U}(\mathcal{M})$ and $W \in \mathrm{U}\left(\mathcal{M}^{\prime}\right)$ are such that $U W$ commutes with $J$, then $U W=U J U J(J U J)^{-1} W$ with $(J U J)^{-1} W \in \mathrm{U}\left(\mathcal{M}^{\prime}\right)_{J}=\operatorname{Inv}(\mathrm{U}(\mathcal{Z}))$ by (ii).

(vi) We shall use the theory of KMS states (cf. [BR96]). We recall that, for a continuous action $\alpha: \mathbb{R} \rightarrow \operatorname{Aut}(\mathcal{A})$ of $\mathbb{R}$ on a $C^{*}$-algebra $\mathcal{A}$, a state $\omega$ of $\mathcal{A}$ is called an $\alpha$-KMS state if, for every pair of hermitian elements $A, B \in \mathcal{A}$, the function

$$
\psi: \mathbb{R} \rightarrow \mathbb{C}, \quad \psi(t):=\omega\left(A \alpha_{t}(B)\right)
$$

extends analyticallty to a holomorphic function on the strip $\mathcal{S}:=\{z \in \mathbb{C}: 0<\operatorname{Im} z<1\}$, extends continuously to its closure and satisfies $\psi(i+t)=\overline{\psi(t)}$ for $t \in \mathbb{R}$.

First we observe that $\omega(A):=\langle\Omega, A \Omega\rangle$ is a KMS state with respect to the modular automorphism group $\alpha_{t}(A)=\Delta^{i t} A \Delta^{-i t}$ (Takesaki's Theorem, [BR96, Thm. 5.3.10]). Now let $\xi \in \operatorname{cs}(\mathcal{M})$ with $V_{\xi}=V_{\Omega}$, i.e., $J_{\xi}=J$ and $\Delta_{\xi}=\Delta$. Then, for the same reason, the state $\omega_{\xi}(A):=\langle\xi, A \xi\rangle$ is also an $\alpha$-KMS state. By [BR96, Prop. 5.3.29], there exists a unique positive selfadjoint operator $T \geq 0$ affiliated with $\mathcal{Z}$ such that

$$
\langle\xi, A \xi\rangle=\omega_{\xi}(A)=\omega(\sqrt{T} A \sqrt{T})=\langle\Omega, \sqrt{T} A \sqrt{T} \Omega\rangle=\langle\sqrt{T} \Omega, A \sqrt{T} \Omega\rangle \quad \text { for } \quad A \in \mathcal{M} .
$$

Therefore $\xi$ and $\sqrt{T} \Omega$ define the same state. Further $\sqrt{T} \Omega$ is also contained in the natural cone $\mathcal{P}$ ([BR87, Prop. 2.5.26]).

As we have seen in (i), there exists a unique $U \in \mathrm{U}\left(\mathcal{M}^{\prime}\right)$ with $U \xi \in \operatorname{cs}(\mathcal{M})_{+}$. As $J=J_{U \xi}=U J_{\xi} U^{-1}=U^{-1} J U$, it follows from (ii) that $U \in \operatorname{Inv}(\mathrm{U}(\mathcal{Z}))$. As $U \xi$ and $\sqrt{T} \Omega$ define the same state and both are contained in $\mathcal{P}$, BR87, Thm. 2.5.31] yields $U \xi=\sqrt{T} \Omega$, i.e., $\xi=U \sqrt{T} \Omega$. Now the assertion follows with $Z:=U \sqrt{T}$.

Suppose, conversely, that $\xi=Z \Omega$ with a selfadjoint operator affiliated to $\mathcal{Z}$. Decomposing $\mathcal{H}, \mathcal{M}$ and $\Omega$ as a direct sum corresponding to bounded spectral projections of $Z$ (which are central in $\mathcal{M}$ as well), we may w.l.o.g. assume that $Z$ is bounded. Since $\xi$ is separating, $\operatorname{ker} Z=\{0\}$, so that we may further assume that $Z$ is invertible. As $Z$ commutes with $J$ and $\Delta$, it commutes with $S=J \Delta^{1 / 2}$, and thus $Z$ leaves $V=\operatorname{Fix}(S)$ invariant. This shows that $V_{\xi}=Z V=V$.

Remark 4.22. (a) Proposition 4.21(iv) describes the fibers of the map

$$
\operatorname{cs}(\mathcal{M}) \rightarrow \operatorname{mc}(\mathcal{M}), \quad \xi \mapsto J_{\xi}
$$

This map is $\mathrm{U}\left(\mathcal{M}^{\prime}\right)$-equivariant, so that the space $\operatorname{cs}(\mathcal{M})$ is a homogeneous $\mathrm{U}\left(\mathcal{M}^{\prime}\right)$-bundle over the symmetric space $\operatorname{mc}(\mathcal{M})$.

(b) Proposition 4.21 (vi) describes the fibers of the $\operatorname{map} \operatorname{cs}(\mathcal{M}) \rightarrow \operatorname{ms}(\mathcal{M})$ in terms of the center $\mathcal{Z}$. If $\mathcal{M}$ is a factor, i.e., $\mathcal{Z}=\mathbb{C} \mathbf{1}$, then we see in particular that $V_{\xi}=V$ implies $\xi= \pm \Omega$ (because $\|\xi\|=1$ ).

LEMma 4.23 (Stabilizer subgroup of $V=V_{\Omega}$ ). The stabilizer of $V$ in the group $G:=$ $\mathrm{U}(\mathcal{M}) \mathrm{U}\left(\mathcal{M}^{\prime}\right)$ consists of all elements of the form $g=u j(u) z$ with $z \in \operatorname{Inv}(\mathrm{U}(\mathcal{Z}))$ and $u \in \mathrm{U}(\mathcal{M})$ fixed by the modular automorphisms $\alpha_{t}(M)=\Delta^{i t} M \Delta^{-i t}$.

Proof. Since standard subspaces are completely determined by their modular objects, 
the stabilizer of $V$ in $G$ is

$$
G_{V}=G_{J} \cap G_{\Delta}=\left\{g \in G: g J g^{-1}=J, g \Delta g^{-1}=\Delta\right\} .
$$

By Proposition 4.21(v), any $g \in G_{J}$ is of the form $g=u j(u) z$ with $u \in \mathrm{U}(\mathcal{M})$ and $z \in$ $\operatorname{Inv}(\mathrm{U}(\mathcal{Z}))$. As $z$ is central, it commutes with the modular unitaries $\Delta^{i t}$ (Theorem $4.2(\mathrm{c})$ ), i.e., $z \in G_{V}$. An element of the form $g=u j(u)$ is fixed by each $\alpha_{t}$ if and only if

$$
\alpha_{t}(u) J \alpha_{t}(u) J=u J u J, \quad \text { resp. } \quad u^{-1} \alpha_{t}(u)=J u \alpha_{t}(u)^{-1} J .
$$

Then $u^{-1} \alpha_{t}(u) \in \mathcal{M} \cap J \mathcal{M} J=\mathcal{Z}$. To see that this implies that $\alpha_{t}(u)=u$, we consider the commutative von Neumann subalgebra $\mathcal{A} \subseteq \mathcal{M}$ generated by the center $\mathcal{Z}$ and $u$. As each $\alpha_{t}$ fixes the center pointwise, we have $\alpha_{t}(\mathcal{A})=\mathcal{A}$ for every $t \in \mathbb{R}$. Then the state of $\mathcal{A}$ given by $\omega(A):=\langle\Omega, A \Omega\rangle$ is a KMS state with respect to $\left.\alpha_{t}\right|_{\mathcal{A}}$, so that the restrictions $\left.\alpha_{t}\right|_{\mathcal{A}}$ are the unique automorphisms corresponding to this KMS states ([BR96. Thm. 5.3.10]). Since $\mathcal{A}$ is abelian, the uniqueness of the automorphism group implies its triviality. We conclude that each $\alpha_{t}$ fixes $u$ if $g \in G_{V}$.

If, conversely, $\alpha_{t}(u)=u$, then $\alpha_{t}$ fixes $g$. This implies that $g$ commutes with $S=$ $J \Delta^{1 / 2}$, hence preserves $V=\operatorname{Fix}(S)$.

EXAmple 4.24. (a) If $\mathcal{M}$ is a factor, then $\operatorname{Inv}(\mathrm{U}(\mathcal{Z}))=\{ \pm 1\}$, so that $\mathrm{U}(\mathcal{M})_{J}=\{ \pm \mathbf{1}\}$ and $\operatorname{mc}(\mathcal{M}) \cong \mathrm{U}(\mathcal{M}) /\{ \pm \mathbf{1}\}$.

(b) For $\mathcal{M}=B(\mathcal{K})$ acting on $\mathcal{H}=B_{2}(\mathcal{K})$ by left multiplications, we have $J A=A^{*}$ (Example 4.6(b)) and by $(\mathrm{a})$, we have $\operatorname{mc}(B(\mathcal{K})) \cong \mathrm{U}(\mathcal{K}) /\{ \pm \mathbf{1}\}$. For any $0<\Omega=\Omega^{*} \in$ $\operatorname{cs}(\mathcal{M})$ we have $\mathcal{P}=\left\{A \in B_{2}(\mathcal{K}): A \geq 0\right\}$.

If $\Omega=\sqrt{D}$ holds for the trace class operator $D>0$, then the centralizer of $\Delta$ in $\mathrm{U}(\mathcal{M})$ is

$$
\left\{g \in \mathrm{U}(\mathcal{M}): g \Delta g^{-1}=\Delta\right\} \cong\left\{g \in \mathrm{U}(\mathcal{K}): g D g^{-1}=D\right\},
$$

and since $D$ is diagonalizable, this subgroup consists of those unitaries leaving all eigenspaces of $D$ invariant. In particular

$$
\left\{A \in B_{2}(\mathcal{K}): A=A^{*},[A, D]=0\right\} \subseteq V \cap V^{\prime}
$$

shows that $V \cap V^{\prime}$ is much larger than $\mathbb{R} \Omega$. For every elements $A=A^{*} \notin \mathcal{P} \cup-\mathcal{P}$ with ker $A=\{0\}$, we have $J A=A$ but $J_{A} \neq J$ (Proposition 4.21(iv)).

(c) For $\mathcal{M}=L^{\infty}(X, \mathfrak{S}, \mu), \mu$ finite, acting on $\mathcal{H}=L^{2}(X, \mu)$ by multiplication operators, we find for $J f=\bar{f}$ that $\mathrm{U}(\mathcal{M})_{J}$ is the set of involutions in $\mathrm{U}(\mathcal{M})$. As the squaring map $U \mapsto U^{2}$ is a morphism of Banach-Lie groups, the Banach symmetric space $\operatorname{mc}(\mathcal{M})$ is diffeomorphic to the unitary group and we have a short exact sequence

$$
\mathbf{1} \rightarrow \operatorname{Inv}(\mathrm{U}(\mathcal{M})) \rightarrow \mathrm{U}(\mathcal{M}) \rightarrow \operatorname{mc}(\mathcal{M}) \rightarrow \mathbf{1}
$$

5. Nets of standard subspaces and von Neumann algebras. In this section we briefly discuss some elementary properties of nets of standard subspaces $\left(V_{\ell}\right)_{\ell \in L}$ and their connection with antiunitary representations $(U, \mathcal{H})$. The connection with nets of von Neumann algebras and QFT is made in \$5.2. Nets of standard subspaces are considerably simpler than nets of von Neumann algebras and naturally determined by an antiunitary representation, of the group generated by all subgroups $U^{V_{\ell}}\left(\mathbb{R}^{\times}\right) \subseteq \mathrm{AU}(\mathcal{H})$ (Proposition 3.2), but this group need not be finite dimensional. 
5.1. Nets of standard subspaces. Let $\mathcal{V}:=\left(V_{\ell}\right)_{\ell \in L}$ be a family of standard subspaces of the Hilbert space $\mathcal{H}$. We assume that the map $\ell \mapsto V_{\ell}$ is injective, so that the index set $L$ is only a notational convenience and we could equally well work directly with the subset $\left\{V_{\ell}: \ell \in L\right\} \subseteq \operatorname{Stand}(\mathcal{H})($ cf. BGL02, SW03]).

Definition 5.1. A net automorphism is an $\alpha \in \mathrm{AU}(\mathcal{H})$ permuting the standard subspaces $V_{\ell}$. We write $\operatorname{Aut}(\mathcal{V}) \subseteq \mathrm{AU}(\mathcal{H})$ for the subgroup of net automorphisms. A net automorphism is called an internal symmetry if it preserves each $V_{\ell}$ separately. The corresponding subgroup of $\operatorname{Aut}(\mathcal{V})$ is denoted $\operatorname{Inn}(\mathcal{V})$.

We write $\left(\Delta_{\ell}, J_{\ell}\right)$ for the modular objects corresponding to $V_{\ell}$ and consider the modular symmetry group

$$
\mathcal{J}:=\operatorname{grp}\left(\left\{J_{\ell}: \ell \in L\right\}\right) \subseteq \operatorname{AU}(\mathcal{H})
$$

generated by the modular conjugations. A natural assumption enriching the underlying geometry is the condition of geometric modular action

$(\mathrm{CGMA}) \mathcal{J} \subseteq \operatorname{Aut}(\mathcal{V})$

(see [BS93, BDFS00] for the von Neumann context). The condition

(MS) $\Delta_{V_{\ell}}^{i t} \in \mathcal{J}$ for all $t \in \mathbb{R}, \ell \in L$

is called the modular stability condition ([BDFS00, [Bo00, $\S \S \mathrm{IV} .5 .6 / 7]$ ).

From now on we assume that (CGMA) is satisfied. We obtain an action $\sigma: \operatorname{Aut}(\mathcal{V}) \times L \rightarrow L,(g, \ell) \mapsto \sigma_{g}(\ell)$ on the index set $L$ by

$$
g V_{\ell}=V_{\sigma_{g}(\ell)} \text {. }
$$

This further implies

$$
g J_{\ell} g^{-1}=J_{\sigma_{g}(\ell)} \quad \text { and } \quad g \Delta_{\ell} g^{-1}=\Delta_{\sigma_{g}(\ell)} .
$$

In particular, (CGMA) implies that

$$
\mathcal{S}:=\left\{J_{\ell}: \ell \in L\right\}
$$

is a conjugation invariant set of generators of $\mathcal{J}$. This fact opens the door to the construction of geometric structures from the group $\mathcal{J}$ and its generating set $\mathcal{S}$ in specific situations.

LEMma 5.2. The subgroup of $\mathcal{J}$ of those elements acting trivially on $L$ is its center

$$
\mathcal{Z}:=\left\{g \in \mathcal{J}:(\forall \ell \in L) g V_{\ell}=V_{\ell}\right\}=\operatorname{Inn}(\mathcal{V}) \cap \mathcal{J}=\operatorname{ker} \sigma .
$$

Proof. For $g \in \mathcal{J}$, the relation $\sigma_{g}=\operatorname{id}_{L}$ implies that $g$ commutes with every element $J_{\ell} \in \mathcal{S}$ by (22). As $\mathcal{J}$ is generated by $\mathcal{S}$, the assertion follows.

By Lemma 5.2, the action of $\mathcal{J}$ on the index set describes this group as a central extension of the group $\mathcal{J} / \mathcal{Z}$ that acts faithfully on the set $L$ which is supposed to carry geometric information (cf. BDFS00, and Remark 5.5 below).

An immediate consequence of (CGMA) is that the net is invariant under the passage to the symplectic orthogonal space $V_{\ell} \mapsto V_{\ell}^{\prime}=J_{\ell} V_{\ell}$ (cf. \$3.2). In particular, we have a duality map $\ell \mapsto \ell^{\prime}:=\sigma_{J_{\ell}}(\ell)$ on $L$. We also have a natural order structure $\leq$ on $L$ by

$$
\ell_{1} \leq \ell_{2} \quad \text { if } \quad V_{\ell_{1}} \subseteq V_{\ell_{2}} .
$$


REMARK 5.3. The key properties of the triple $\left(L, \leq,^{\prime}\right)$, given by the partial order $\leq$ and the duality map $\ell \mapsto \ell^{\prime}$ are that

(A1) $\ell_{1} \leq \ell_{2}$ implies $\ell_{2}^{\prime} \leq \ell_{1}^{\prime}$, and

(A2) $\ell_{1} \leq \ell_{2}^{\prime}$ if and only if $\ell_{2} \leq \ell_{1}^{\prime}$.

From (A2) we immediately derive $\ell \leq \ell^{\prime \prime}$ and by combining this with (A1), we obtain $\ell^{\prime}=\ell^{\prime \prime \prime}$ for every $\ell \in L$, i.e., the duality map restricts to an involution on its range.

EXAmples 5.4. (a) For a subset $S$ of the Minkowski space $\mathbb{R}^{1, d-1}$, we define the causal complement by

$$
S^{\prime}:=\left\{x \in \mathbb{R}^{1, d-1}:(\forall y \in S)[x-y, x-y]<0\right\} .
$$

Then $S^{\prime}=\bigcap_{s \in S}\{s\}^{\prime}$, which immediately leads to (A1/2). Here $S \subseteq T^{\prime}$ means that $S$ and $T$ are space-like separated, and $S^{\prime \prime}$ is the causal completion of $S$. For $g \in P(d)$ and $S \subseteq \mathbb{R}^{1, d-1}$, we have $(g S)^{\prime}=g S^{\prime}$.

For the standard right wedge $W_{R} \subseteq \mathbb{R}^{1, d-1}$, we have $W_{R}^{\prime}=-\overline{W_{R}}$ and $W_{R}^{\prime \prime}=W_{R}$, and for the positive light cone $V_{+}$we have $V_{+}^{\prime}=\emptyset$ and $V_{+}^{\prime \prime}=\mathbb{R}^{1, d-1}$. For $x-y \in V_{+}$, the causal completion

$$
\{x, y\}^{\prime \prime}=\left(x-\overline{V_{+}}\right) \cap\left(y+\overline{V_{+}}\right)=\overline{\mathcal{O}_{x, y}}
$$

is the closure of the double cone $\mathcal{O}_{x, y}$ (cf. Remark 5.13(b)).

(b) If $(X, \leq)$ is a partially ordered space, then we define

$$
\{x\}^{\prime}:=\{y \in X: x \not \leq y, y \not \leq x\} \quad \text { and } \quad S^{\prime}:=\bigcap_{s \in S}\{s\}^{\prime} .
$$

Then the set $L$ of subsets of $X$, endowed with the inclusion order, satisfies $(\mathrm{A} 1 / 2)$.

(c) For a complex Hilbert space $\mathcal{H}$, the set of real subspace $V \subseteq \mathcal{H}$, endowed with the inclusion order and the symplectic orthogonal space $V^{\prime}=i V^{\perp \mathbb{R}}$ satisfies $(\mathrm{A} 1 / 2)$.

(d) For a complex Hilbert space $\mathcal{H}$, the set of von Neumann subalgebras $\mathcal{M} \subseteq B(\mathcal{H})$, endowed with the inclusion order and the commutant map $\mathcal{M} \mapsto \mathcal{M}^{\prime}$ satisfies $(\mathrm{A} 1 / 2)$.

REMARK 5.5. (a) In QFT, one expects that the structure $\left(L, \leq,,^{\prime}\right)$ encodes physical information and one would like to recover information on the geometry of spacetime from this structure. In this context, causal complements, resp., the notion of being spacelike separated, appears more fundamental than the causal order if we want to recover a spacetime $M$ from the triple $\left(L, \leq,^{\prime}\right)$, where $L$ consists of certain subsets of $M$ but does not contain one-point sets (cf. Ke96]).

If the modular stability condition is satisfied, i.e., if $\mathcal{J}$ contains also the modular unitaries, this group is supposed to encode the dynamics of the quantum theory, the isometry group of the corresponding spacetime and a (projective) unitary representation of this group ([Su05, §6.4]). This connects naturally with the approach of Connes and Rovelli who "construct" the dynamics of a quantum statistical system by a modular one-parameter group $\Delta^{i t}([\mathrm{CR} 94])$.

Here an interesting result concerning the detection of known group from this viewpoint is the characterization of the Poincaré group in terms of structure preserving maps on the set $\mathcal{W}$ of wedges in $\mathbb{R}^{1, d-1}$ (BDFS00, [Bo00, §IV.5], Lemma 4.12). 
(b) In [Ke98, §4] the relation of the causal structure on spacetime and how it can be determined by data encoded in nets of $C^{*}$-algebras is discussed very much in the spirit of this section. We refer to Ra17] for an approach to quantum field theory based on modular theory of operator algebras that does not assume an a priori given underlying spacetime. Instead, one would like to generate the spacetime geometry from operator theoretic data.

(c) In SW03 this program is carried out to a large extent by specifying a set of axioms formulated in terms of the modular conjugations $J_{\ell}$, such that the index set $L$ corresponds to the set $\mathcal{W}$ of wedges in three-dimensional Minkowski space $\mathbb{R}^{1,2}, J_{W}$ corresponds to the the orthogonal reflection $r_{W} \in P(3)_{+}$in the edge of $W$ and $\mathcal{J} \cong P(3)_{+}$(cf. Lemma 4.12).

In this case, the subset $\mathrm{SO}_{1,2}(\mathbb{R})^{\uparrow} W_{R} \subseteq \mathcal{W}$ identifies naturally with the anti-deSitter space $\mathrm{AdS}^{2} \cong \mathrm{SO}_{1,2}(\mathbb{R})^{\uparrow} / \mathrm{SO}_{1,1}(\mathbb{R})^{\uparrow}$, which can be realized as an adjoint orbit in the Lie algebra $\mathfrak{s o}_{1,2}(\mathbb{R}) \cong \mathfrak{s l}_{2}(\mathbb{R})$ (cf. Lemma 4.12(v)).

The following construction is of fundamental importance in our approach. It is inspired by the modular localization approach to QFT developed in [BGL02, Thm. 2.5]:

Proposition 5.6. (Nets of standard subspaces from antiunitary representations; the BGL construction) Let $(U, \mathcal{H})$ be an antiunitary representation of the Lie group pair $\left(G, G_{1}\right)$ and associate to $\gamma:\left(\mathbb{R}^{\times}, \mathbb{R}_{+}^{\times}\right) \rightarrow\left(G, G_{1}\right)$ the standard subspace $V_{\gamma}$ with $U^{V_{\gamma}}=$ $U \circ \gamma$ (Proposition 3.2$)$. Then, for every non-empty subset $\Gamma \subseteq \operatorname{Hom}\left(\left(\mathbb{R}^{\times}, \mathbb{R}_{+}^{\times}\right),\left(G, G_{1}\right)\right)$ invariant under conjugation with elements of $G$ and under inversion, we thus obtain a net $\left(V_{\gamma}\right)_{\gamma \in \Gamma}$ of standard subspaces which satisfies (CGMA) for the group $\mathcal{J}$ which is the image under $U$ of the subgroup of $G$ generated by the conjugation invariant set of involutions $\{\gamma(-1): \gamma \in \Gamma\}$.

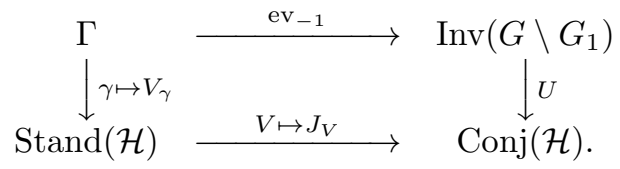

Proof. This follows from the observation that, for $\gamma^{g}(t):=g \gamma(t) g^{-1}$, we have $U_{g} V_{\gamma}=V_{\gamma^{g}}$ for $g \in G_{1}$ and $U_{g} V_{\gamma}=V_{\gamma^{g}}^{\prime}$ for $g \notin G_{1}$, so that $G$ acts naturally by automorphisms on the net $\left(V_{\gamma}\right)_{\gamma \in \Gamma}$.

REMARK 5.7. (a) Evaluation in -1 leads to a fibration

$$
\mathrm{ev}-1_{1}: \operatorname{Hom}\left(\left(\mathbb{R}^{\times}, \mathbb{R}_{+}^{\times}\right),\left(G, G_{1}\right)\right) \rightarrow \operatorname{Inv}\left(G \backslash G_{1}\right) .
$$

An involution $r \in G \backslash G_{1}$ is contained in the image if and only if there exists an $x \in \mathfrak{g}$ fixed by $\operatorname{Ad}(r)$, i.e., if $\mathfrak{g}^{\operatorname{Ad}(r)}=\operatorname{ker}(\operatorname{Ad}(r)-\mathbf{1}) \neq\{0\}$. This is always the case if $\mathfrak{g}$ is non-abelian, i.e., if $-\mathrm{id}_{\mathfrak{g}}$ is not an automorphism. Then the fiber over $r$ can be identified with the Lie subalgebra $\mathfrak{g}^{\operatorname{Ad}(r)}$.

(b) In many situations one considers minimal sets

$$
\Gamma=C_{\gamma} \cup C_{\gamma^{\vee}}, \quad \text { where } \quad C_{\gamma}:=\left\{\gamma^{g}: g \in G\right\} .
$$

Then ev-1 $\left(C_{\gamma}\right)=C_{r}$ is the conjugacy class of the involution $r:=\gamma(-1) \in G \backslash G_{1}$, hence in particular a symmetric space (cf. Appendix A.3). An important example in QFT is $\gamma=\gamma_{W_{R}}$ for $G=P(d)_{+}($Lemma 4.12) . 
(c) In the context of Proposition 5.6, the relation $V_{\gamma}^{\prime}=V_{\gamma} \vee$ shows that duality is naturally built into the construction. However, in general it may not be so easy to determine when $V_{\gamma_{1}} \subseteq V_{\gamma_{2}}$. In [BGL02, Thm. 3.4] it is shown that, for $G=P(d)_{+}$and homomorphisms $\left(\gamma_{W}\right)_{W \in \mathcal{W}}$ corresponding to wedges, the relation $W_{1} \subseteq W_{2}$ is equivalent to $V_{\gamma_{W_{1}}} \subseteq V_{\gamma_{W_{2}}}$ if and only if $U$ is a positive energy representation.

The preceding discussion suggests a closer look at conjugacy classes of involutions $\tau \in G \backslash G_{1}$. We write $C_{\tau} \subseteq G$ for the conjugacy class of $G$.

Lemma 5.8. Let $G_{1}$ be a connected Lie group with Lie algebra $\mathfrak{g}$ and $\tau \in \operatorname{Aut}\left(G_{1}\right)$ be an involutive automorphism. Then the conjugacy class $C_{\tau} \subseteq G:=G_{1} \rtimes\{\mathbf{1}, \tau\}$ generates the subgroup $\operatorname{grp}\left(C_{\tau}\right)=B\{\mathbf{1}, \tau\}$, where $B$ is the integral subgroup whose Lie algebra is the ideal $\mathfrak{b}:=\mathfrak{g}^{-\tau}+\left[\mathfrak{g}^{-\tau}, \mathfrak{g}^{-\tau}\right]$. In particular, $C_{\tau}$ generates $G$ if and only if $\mathfrak{g}^{\tau}=\left[\mathfrak{g}^{-\tau}, \mathfrak{g}^{-\tau}\right]$.

Proof. Let $H=\operatorname{grp}\left(C_{\tau}\right) \subseteq G$ be the subgroup generated by $C_{\tau}$. As $\tau \in H$, we have $H=B\{e, \tau\}$ for $B:=H \cap G_{1}$. Then $B$ is generated by the elements of the form $g \tau(g)^{-1}$, $g \in G_{1}$, hence in particular arcwise connected. For $x \in \mathfrak{g}^{-\tau}$, we therefore obtain $\exp (2 x)=$ $\exp (x) \tau(\exp (-x)) \in B$, so that the Lie algebra $\mathfrak{b}$ of $B$ contains $\mathfrak{g}^{-\tau}$ and hence also $\mathfrak{b}=\mathfrak{g}^{-\tau}+\left[\mathfrak{g}^{-\tau}, \mathfrak{g}^{-\tau}\right]$, which is an ideal of $\mathfrak{g}_{1}$.

Let $\widetilde{G}_{1}$ denote the universal covering group of $G_{1}$. Then $\widetilde{B}:=\left\langle\exp _{\widetilde{G}_{1}} \mathfrak{b}\right\rangle$ is a normal integral subgroup of $\widetilde{G}_{1}$, hence closed. As $\tau$ acts trivially on the quotient group $\widetilde{G}_{1} / \widetilde{B}$, all elements of the form $g \tau(g)^{-1}$ are contained in $\widetilde{B}$. Therefore $B:=\left\langle\exp _{G} \mathfrak{b}\right\rangle$ contains the arcwise connected subgroup $H \cap G_{1}$, and thus $H \cap G_{1}=B$. This implies the lemma.

ExAmple 5.9. $G=\operatorname{Aff}(\mathbb{R})$ and $\tau=(x,-1)$ with $C_{\tau}=\mathbb{R} \times\{-1\}$, and this conjugacy class generates $G$.

LEMMA 5.10. Let $\tau=r_{W} \in P(d)_{+}$be a wedge reflection for some $W \in \mathcal{W}$. Then

(i) The conjugacy class $C_{\tau}$ of $\tau$ generates $P(d)_{+}$if and only if $d>2$.

(ii) The conjugacy class $C_{\tau}$ of $\tau$ in the conformal group $\mathrm{SO}_{2, d}(\mathbb{R})$ generates the whole group for any $d>0$.

Proof. (i) Since all wedges $W \in \mathcal{W}$ are conjugate to the standard right wedge $W_{R}$, it suffices to consider $\tau=r_{W_{R}}=R_{01}=\operatorname{diag}(-1,-1,1, \ldots, 1)$. If $d=2$, then $R_{01}=-\mathbf{1}$, so that $\operatorname{grp}\left(C_{\tau}\right)=\mathbb{R}^{2} \rtimes\{ \pm \mathbf{1}\}$ is a proper subgroup of $P(2)_{+}$.

The case $d=2$ already implies that $\operatorname{grp}\left(C_{\tau}\right)$ contains all translations in the directions of all Lorentzian 2-planes, hence all translations. Therefore it suffices to show that the conjugacy class of $R_{01}$ in $\mathrm{SO}_{1, d}(\mathbb{R})$ generates the whole group. In view of Lemma 5.8, this follows from the simplicity of the real Lie algebra $\mathfrak{g}=\mathfrak{s o}_{1, d-1}(\mathbb{R})$.

(ii) We consider $\mathrm{SO}_{2, d}(\mathbb{R})$ as a group acting on $\mathbb{R}^{1, d-1}$ by rational maps (cf. HN12, $\S 17.4])$. We have already seen above that the group $\operatorname{grp}\left(C_{\tau}\right)$ generated by the conjugacy class $C_{\tau}$ in $\mathrm{SO}_{2, d}(\mathbb{R})$ contains the Poincaré group $P(d)_{+}$, which is a parabolic subgroup of $\mathrm{SO}_{2, d}(\mathbb{R})$ and it intersects both connected components. By the same argument, it contains the opposite parabolic subgroup, and both subgroups generate $\mathrm{SO}_{2, d}(\mathbb{R})$ because it has only two connected components (cf. [Be96]).

If $d$ is odd, then $\mathrm{SO}_{2, d}(\mathbb{R}) \cong \mathrm{O}_{2, d}(\mathbb{R}) /\{ \pm \mathbf{1}\}$ is the full conformal group of $\mathbb{R}^{1, d-1}$, but if $d$ is even, then the kernel $\{ \pm \mathbf{1}\}$ of the action of $\mathrm{O}_{2, d}(\mathbb{R})$ is contained in the identity 
component, so that $\operatorname{Conf}\left(\mathbb{R}^{1, d}\right) \cong \mathrm{O}_{2, d}(\mathbb{R}) /\{ \pm \mathbf{1}\}$ has four connected components ( $\S 17.4])$. Therefore the conjugacy class of a wedge reflection does not generate the whole conformal group.

Remark 5.11. In [BGL02, Thm. 4.7], Brunetti, Guido and Longo describe a one-to-one correspondence between antiunitary positive energy representations of $P(d)_{+}$and certain nets of closed real subspaces $V_{\mathcal{O}}$ indexed by certain open subsets $\mathcal{O} \subseteq \mathbb{R}^{d}$, for which the subspaces $\left(V_{W}\right)_{W \in \mathcal{W}}$ corresponding to wedges are standard and the modular covariance condition

$$
\Delta_{W}^{-i t / 2 \pi} V_{\mathcal{O}}=V_{\gamma_{W}(t) \mathcal{O}}
$$

holds for the homomorphisms $\gamma_{W}: \mathbb{R}^{\times} \rightarrow P(d)_{+}$and the modular unitaries of $V_{W}$.

The uniqueness of the local net, once the unitary representation is given, is discussed in [BGL02, Rem. 4.8] (see also BGL93]). For the converse, i.e., the uniqueness of the unitary representation, once the local net is given, we refer to BGL93. In Mu01, Mund shows that, for any representation $(U, \mathcal{H})$ of $P(d)_{+}^{\uparrow}$ that is a finite direct sum of irreducible representations of strictly positive mass, there is only one covariant net of standard subspaces; which therefore coincides with the one obtained in Proposition 5.6 from any antiunitary extension of $U$ to $P(d)_{+}$.

Example 5.12. (Nets arising from a single von Neumann algebra)

(a) Let $\mathcal{M} \subseteq B(\mathcal{H})$ be a von Neumann algebra for which $\operatorname{cs}(\mathcal{M}) \neq \emptyset$ and consider the corresponding set

$$
\mathcal{V}:=\operatorname{ms}(\mathcal{M})=\left\{V_{\xi}: \xi \in \operatorname{cs}(\mathcal{M})\right\}
$$

of standard subspaces (Definition 4.20 ). Fix fix a cyclic separating vector $\Omega$ and the corresponding modular objects $(\Delta, J)$ and consider the group

$$
G:=\mathrm{U}(\mathcal{M}) \mathrm{U}\left(\mathcal{M}^{\prime}\right)\{\mathbf{1}, J\} \subseteq \mathrm{AU}(\mathcal{H}) .
$$

It is easy to see that this group permutes the standard subspaces in $\mathcal{V}$. From Proposition 4.21 (ii) we derive that

$$
\operatorname{mc}(\mathcal{M})=\left\{g J g^{-1}: g \in G\right\}
$$

is the conjugacy class of $J$ in $G$. We also note that the $G$-orbit $\left\{g \mathcal{M} g^{-1}: g \in G\right\}=$ $\left\{\mathcal{M}, \mathcal{M}^{\prime}\right\}$ of $\mathcal{M}$ in the set of von Neumann subalgebras of $B(\mathcal{H})$ consists only of two elements.

(b) Consider the group

$$
G^{\sharp}:=\mathrm{U}(\mathcal{M}) \mathrm{U}\left(\mathcal{M}^{\prime}\right) \gamma\left(\mathbb{R}^{\times}\right) \quad \text { for } \quad \gamma(-1):=J \quad \text { and } \quad \gamma\left(e^{t}\right):=\Delta^{-i t / 2 \pi} .
$$

That $G^{\sharp}$ is a group follows from the fact that $\gamma\left(\mathbb{R}_{+}^{\times}\right)$normalizes $\mathrm{U}(\mathcal{M})$ and $\mathrm{U}\left(\mathcal{M}^{\prime}\right)$, whereas conjugation by $J=\gamma(-1)$ exchanges both. This group is strictly larger than $\mathrm{U}(\mathcal{M}) \mathrm{U}\left(\mathcal{M}^{\prime}\right)\{\mathbf{1}, J\}$ if the modular automorphisms $\alpha_{t}(M):=\Delta^{i t} M \Delta^{-i t}$ of $\mathcal{M}$ are not inner.

If $\xi \in \operatorname{cs}(\mathcal{M})$ is different from $\Omega$, then Connes' Radon Nikodym Theorem 5 implies the existence of a strongly continuous path of unitaries $\left(u_{t}\right)_{t \in \mathbb{R}}$ in $\mathrm{U}(\mathcal{M})$ such that the

\footnotetext{
${ }^{5}$ See [Bla06, Thm. III.4.7.5], BR96, Thm. 5.3.34], and in particular [F198 for a quite direct proof.
} 
corresponding modular automorphism group $\alpha_{t}^{\xi}(M)=\Delta_{\xi}^{i t} M \Delta_{\xi}^{-i t}$ satisfies

$$
\alpha_{t}^{\xi}(M)=u_{t} \alpha_{t}(M) u_{t}^{*} \quad \text { for } \quad M \in \mathcal{M}, t \in \mathbb{R} .
$$

This implies that $\Delta_{\xi}^{-i t} u_{t} \Delta^{i t} \in \mathrm{U}\left(\mathcal{M}^{\prime}\right)$, so that $G^{\sharp}$ also contains the operators $\Delta_{\xi}^{i t}$. Hence the net of standard subspaces of $\mathcal{H}$ specified by the conjugacy class of the antiunitary representation $\gamma \in \operatorname{Hom}\left(\mathbb{R}^{\times}, G^{\sharp}\right)$ coincides with the orbit $G^{\sharp} V=G V \subseteq \operatorname{Stand}(\mathcal{H})$.

5.2. Nets of von Neumann algebras. The context that actually motivates the consideration of families of standard subspaces are families $\left(\mathcal{M}_{\ell}\right)_{\ell \in L}$ of von Neumann algebras on some Hilbert space $\mathcal{H}$. In the theory of algebras of local observables, one considers $\ell$ as indicating the "laboratory" in which observables corresponding to $\mathcal{M}_{\ell}$ can be measured, and then $L$ is the set of laboratories (cf. Ha96, Ar99, Bo97]).

We write $\mathcal{M} \subseteq B(\mathcal{H})$ for the von Neumann algebra generated by all the algebras $\mathcal{M}_{\ell}$. We shall discuss several properties of these families and relate them to antiunitary representations and some results in Algebraic Quantum Field Theory (AQFT). Our first assumption is the Reeh-Schlieder property:

(RS) There exists a unit vector $\Omega$ that is cyclic and separating for each $\mathcal{M}_{\ell}$.

By the Tomita-Takesaki Theorem, (RS) leads to a family of standard subspaces given

$$
V_{\ell}:=\overline{\mathcal{M}_{\ell, h} \Omega}
$$

and the map $\ell \mapsto V_{\ell}$ is injective if and only if the map $\ell \mapsto \mathcal{M}_{\ell}$ is injective (Lemma 4.7). This leads us to the setting of the preceding subsection, so that everything said there applies in particular here. As each $J_{\ell}$ fixes $\Omega$, it is fixed by the whole group $\mathcal{J}$. For $g \in \mathcal{J}$ and $\mathcal{M}_{\ell}^{g}:=g \mathcal{M}_{\ell} g^{-1}$, we therefore have $g V_{\ell}=\overline{\mathcal{M}_{\ell, h}^{g} \Omega}$, so that Lemma 4.7 implies that $g V_{\ell}=V_{\widetilde{\ell}}$ for some $\tilde{\ell} \in L$ is equivalent to $g \mathcal{M}_{\ell} g^{-1}=\mathcal{M}_{\widetilde{\ell}}$. Hence the condition of geometric modular action (CGMA) from the preceding section is equivalent to the following ([BDFS00]):

(CGMA) Conjugation with elements of the group $\mathcal{J}$ permutes the von Neumann algebras $\left(\mathcal{M}_{\ell}\right)_{\ell \in L}$.

The relation $J_{\ell} \mathcal{M}_{\ell} J_{\ell}=\mathcal{M}_{\ell}^{\prime}$ then implies that the net $\left(\mathcal{M}_{\ell}\right)_{\ell \in L}$ is invariant under the passage to the commutant.

REMARK 5.13. (a) In quantum field theory, where $L$ often is the set $\mathcal{W}$ of wedges in Minkowski space $\mathbb{R}^{1,3}$, BDFS00, Thm. 5.2.6] asserts that (CGMA) basically is equivalent to the duality condition $\mathcal{M}\left(W^{\prime}\right)=\mathcal{M}(W)^{\prime}$ for every $W \in \mathcal{W}$. Then one obtains an antiunitary representation of the Poincaré group $P(4)_{+}$fixing $\Omega$ and acting covariantly on the net. Further, $U_{r_{W}}=J_{W}$ (cf. Lemma 4.12) and the spectrum of the translation subgroup is either contained in $\overline{V_{+}}$or in $-\overline{V_{+}}$, i.e., we either have positive or negative energy representations.

(b) For $x, y \in \mathbb{R}^{1,3}$ and $x-y \in V_{+}$, the open causal interval

$$
\mathcal{O}_{x, y}:=\left(x-V_{+}\right) \cap\left(y+V_{+}\right)
$$

is called a double cone. There are various Reeh-Schlieder Theorems, that provide sufficient conditions for the vacuum vector to be cyclic and separating for an algebras $\mathcal{M}(\mathcal{O})$ 
of local observables attached to an open subset of Minkowski space ([Bo92, RS61, Bo68). The most classical results concern the cyclicity of the vacuum for double cone algebras $\mathcal{M}\left(\mathcal{O}_{x, y}\right)$. Since every wedge contains double cones, the vacuum is also cyclic and separating for wedge algebras $\mathcal{M}(W), W \in \mathcal{W}$. This leads to modular objects $\left(\Delta_{W}, J_{W}\right)$, so that the condition $(\mathrm{RS})$ in $\$ 5.2$ holds for the index set $L=\mathcal{W}$.

(c) For nets of von Neumann algebras $\mathcal{M}(\mathcal{O})$ of local oberservables associated to regions $\mathcal{O}$ in some spacetime $M$, it is important to specify those regions behaving well with respect to our assumptions. In [Sa97] they are called test regions. This requires in particular that the vacuum vector $\Omega$ should be cyclic for $\mathcal{M}(\mathcal{O})$ (the Reeh-Schlieder property) and that a suitable duality holds $\mathcal{M}(\mathcal{O})^{\prime}=\mathcal{M}\left(\mathcal{O}^{\prime}\right)$, where $\mathcal{O}^{\prime}$ is the (interior of the) causal complement of $\mathcal{O}$. Prototypical examples of test domains are wedges $W$ in Minkowski space (or its conformal completion) [BGL02, Thm. 2.5], but in many situations larger classes also have these properties, such as double cones or spacelike cones, i.e., translates of convex cones $\mathbb{R}_{+} \mathcal{D}$, where $\mathcal{D}$ is a double cone not containing 0 . In this context the CGMA is a natural additional requirement for test regions that ties the corresponding modular structure to spacetime geometry.

(d) For a Haag-Kastler net $\mathcal{A}(\mathcal{O})$ (as in the introduction), the (CGMA) for the net $\pi_{\omega}(\mathcal{A}(\mathcal{O}))^{\prime \prime}$ of von Neumann algebras specified by a state $\omega$ of $\mathcal{A}$ can be seen as a requirement that selects states which are particularly natural (cf. BDFS00, p. 485]).

Under certain assumptions on the corresponding net of local observables, the Bisognano-Wichmann Theorem ([BW76, So10] $)$ asserts that the antiunitary representation $(U, \mathcal{H})$ of $P(4)_{+}$obtained from the PCT Theorem, where $\Theta=U_{-1}$ is the antiunitary PCT operator, has the property that the boost generator $b_{0}$ from (5) in Example 2.27 satisfies

$$
\Delta_{W_{R}}^{-i t / 2 \pi}=U\left(e^{t b_{0}}\right) \quad \text { and } \quad J_{W_{R}}=U\left(r_{W_{R}}\right)=\Theta U(\operatorname{diag}(1,1,-1,-1)) .
$$

The first relation is called the modular covariance relation (cf. [Mu01, p. 911]). In GL95, Props. 2.8,2.9] Guido and Longo show that modular covariance implies covariance of the corresponding modular conjugations which in turn implies the PCT Theorem. In the context of standard subspaces, the Bisognano-Wichmann Theorem and the PCT Theorem were derived in by Mund ([Mu01, Thm. 5]).

ExAmples 5.14. (Conformal invariance) Beyond the Bisognano-Wichmann Theorem, the following geometric implementations of modular automorphism groups are known:

(a) In Bu78, Buchholz shows that, for a free scalar massless field on $\mathbb{R}^{1, d-1}$ (which automatically enjoys conformal symmetry), for $d>2$ the dilation group $\gamma_{V_{+}}(a)(x)=$ $a x, a \in \mathbb{R}^{\times}$, corresponds to the modular objects of the light cone algebra $\mathcal{M}\left(V_{+}\right)$. As we shall see in (b) below, the light cone is conformally equivalent to the right wedge $W_{R}$. Therefore $\gamma_{V_{+}}$is conjugate in the conformal group to the homomorphism $\gamma_{W_{R}}: \mathbb{R}^{\times} \rightarrow \operatorname{Conf}\left(\mathbb{R}^{1, d-1}\right)$, corresponding to the right wedge $W_{R}$, which occurs in the Bisognano-Wichmann Theorem.

(b) In HL82, Hislop and Longo obtain similar results for double cones in the context of massless scalar fields by conjugating them conformally to light cones and then apply 
Bu78. More concretely, the relativistic ray inversion

$$
\rho: x=(t, \mathbf{x}) \mapsto \frac{1}{[x, x]}(t, \mathbf{x}), \quad[x, x]=t^{2}-\mathbf{x}^{2}
$$

(which is an involution), exchanges the translated right wedge

$$
W_{R}+\frac{r}{2} e_{1}=\left\{\left(x_{0}, \mathbf{x}\right): x_{1}>\frac{r}{2}+\left|x_{0}\right|\right\}
$$

with the double cone

$$
\mathcal{O}_{\frac{e_{0}-e_{1}}{r}, \frac{-e_{0}-e_{1}}{r}}=\left(\frac{e_{0}-e_{1}}{r}-V_{+}\right) \cap\left(-\frac{e_{0}+e_{1}}{r}+V_{+}\right) .
$$

It also exchanges the double cone $\mathcal{O}_{r e_{0}, 0}=\left(r e_{0}-V_{+}\right) \cap V_{+}$and the light cone $\frac{e_{0}}{r}+V_{+}$(see Gu11, p. 111]). With these explicit transformations, one also obtains the corresponding one-parameter groups of automorphisms and the corresponding conformal involutions. For the light cone $V_{+}$, we know from (a) that the corresponding automorphism group is given by the dilations $\gamma_{V_{+}}(t) x=t x$. So it follows in particular, that it is conformally conjugate to the Lorentz boosts $\gamma_{W}: \mathbb{R}^{\times} \rightarrow P(4)_{+}$corresponding to a wedge $W$ (Lemma 4.12).

As a consequence of this discussion, the modular automorphism groups corresponding to the local observable algebras associated to double cones, light cones and wedges are conjugate under the conformal group $\operatorname{Conf}\left(\mathbb{R}^{1, d-1}\right) \cong \mathrm{O}_{2, d}(\mathbb{R}) /\{ \pm \mathbf{1}\}$. In particular, they correspond to a single conjugacy class of homomorphism $\gamma: \mathbb{R}^{\times} \rightarrow \operatorname{Conf}\left(\mathbb{R}^{1, d-1}\right)$ which is most simply represented by $\gamma_{V_{+}}$.

Example 5.15. (cf. Example 2.28) In the one-dimensional Minkowski space $\mathbb{R}$, the order intervals are represented by the open interval $(-1,1)$ transformed by the Cayley map $c(x):=\frac{1+x}{1-x}$ to $(0, \infty)=V_{+}$and the involution $\sigma(x):=x^{-1}$ maps $(-1,1)$ to its (conformal) complement. These are the geometric transformations corresponding to the modular operators on the double cone algebra $\mathcal{M}\left(\mathcal{O}_{1,-1}\right)$ for $d=1$.

EXAMPLE 5.16. Interesting examples of nets of von Neumann algebras with (CGMA) arise from [BDFS00, Thm. 4.3.9], where the index set is the set $\mathcal{W}$ of wedges in $\mathbb{R}^{1,3}$. Under suitable continuity assumptions, one obtains a continuous antiunitary representation of $P(4)_{+}$with

$$
U_{r_{W}}=J_{W} \quad \text { and } \quad \mathcal{J}=U_{P(4)_{+}} .
$$

Here a key point is that $P(4)_{+}$is generated by the conjugacy class of the wedge reflection $r_{W_{R}}$ (Lemma 5.10).

REMARK 5.17. A key observation in the work of Borchers and Wiesbrock is that von Neumann algebras of local observables corresponding to two wedges having a light ray in common define modular intersections ([Wi98, Prop. 7]). That one can deal with them as pairs without any direct reference to the intersection (cf. Theorem 3.23) is crucial because the modular group of the intersection need not be implemented geometrically Bo96. This is of particular interest for QFT on de Sitter space d $\mathrm{S}^{d}$ whose isometry group $\mathrm{O}_{1, d}(\mathbb{R})$ has no positive energy representations for $d>2$. 
6. Second quantization and modular localization. In this section we explain how Second Quantization, i.e., the passage from a (one-particle) Hilbert space $\mathcal{H}$ to the corresponding Fock spaces $\mathcal{F}_{ \pm}(\mathcal{H})$ (bosonic and fermionic) provides for each standard subspace $V \subseteq \mathcal{H}$ pairs $\left(\mathcal{R}^{ \pm}(V), \Omega\right)$, where $\mathcal{R}^{ \pm}(V)$ is a von Neumann algebra on $\mathcal{F}_{ \pm}(\mathcal{H})$ and the vacuum vector $\Omega \in \mathcal{F}_{ \pm}(\mathcal{H})$ is cyclic.

Let $\mathcal{H}$ be a complex Hilbert space and let

$$
\mathcal{F}(\mathcal{H}):=\widehat{\bigoplus}_{n=0}^{\infty} \mathcal{H}^{\widehat{\otimes} n}
$$

be the full Fock space over $\mathcal{H}$. We write $\mathcal{F}_{+}(\mathcal{H})$ for the subspace of symmetric tensors, the bosonic Fock space, and $\mathcal{F}_{-}(\mathcal{H})$ for the subspace of skew-symmetric tensors, the fermionic Fock space. Both spaces carry a natural representation

$$
\Gamma_{ \pm}: \operatorname{AU}(\mathcal{H}) \rightarrow \operatorname{AU}\left(\mathcal{F}_{ \pm}(\mathcal{H})\right)
$$

of the antiunitary group $\mathrm{AU}(\mathcal{H})$ given by

$$
\Gamma_{+}(U)\left(v_{1} \vee \cdots \vee v_{n}\right):=U v_{1} \vee \cdots \vee U v_{n}, \quad \Gamma_{-}(U)\left(v_{1} \wedge \cdots \wedge v_{n}\right):=U v_{1} \wedge \cdots \wedge U v_{n} .
$$

Moreover, the bosonic Fock space carries a unitary representation of the Heisenberg group Heis $(\mathcal{H})$ 6.1) and its subgroups can be used to derive a net of von Neumann algebras on $\mathcal{F}_{+}(\mathcal{H})$. A similar construction can be carried out for the fermionic Fock space in terms of the natural representation of the $C^{*}$-algebra $\operatorname{CAR}(\mathcal{H})$, a $C^{*}$-algebra defined by the canonical anticommutation operators. Both constructions are functorial and associate to every antiunitary representation $(U, \mathcal{H})$ of $\left(G, G_{1}\right)$ on $\mathcal{H}$ a covariant family $\left(\mathcal{M}_{\gamma}\right)_{\gamma \in \Gamma}$ of von Neumann algebras on $\mathcal{F}_{ \pm}(\mathcal{H})$, where $\Gamma$ is as in Proposition 5.6 .

6.1. Bosonic Fock space. We start with the construction of the von Neumann algebras on the bosonic Fock space. For $v_{1}, \ldots, v_{n} \in \mathcal{H}$, we define

$$
v_{1} \cdots v_{n}:=v_{1} \vee \cdots \vee v_{n}:=\frac{1}{\sqrt{n !}} \sum_{\sigma \in S_{n}} v_{\sigma(1)} \otimes \cdots \otimes v_{\sigma(n)}
$$

and $v^{n}:=v^{\vee n}$, so that

$$
\left\langle v_{1} \vee \cdots \vee v_{n}, w_{1} \vee \cdots \vee w_{n}\right\rangle=\sum_{\sigma \in S_{n}}\left\langle v_{\sigma(1)}, w_{1}\right\rangle \cdots\left\langle v_{\sigma(n)}, w_{m}\right\rangle
$$

For every $v \in \mathcal{H}$, the series $\operatorname{Exp}(v):=\sum_{n=0}^{\infty} \frac{1}{n !} v^{n}$ defines an element in $\mathcal{F}_{+}(\mathcal{H})$ and the scalar product of two such elements is given by

$$
\langle\operatorname{Exp}(v), \operatorname{Exp}(w)\rangle=\sum_{n=0}^{\infty} \frac{n !}{(n !)^{2}}\langle v, w\rangle^{n}=e^{\langle v, w\rangle} .
$$

These elements span a dense subspace of $\mathcal{F}_{+}(\mathcal{H})$, and therefore we have for each $x \in \mathcal{H}$ a unitary operator on $\mathcal{F}_{+}(\mathcal{H})$ determined by the relation

$$
U_{x} \operatorname{Exp}(v)=e^{-\langle x, v\rangle-\frac{\|x\|^{2}}{2}} \operatorname{Exp}(v+x) \quad \text { for } \quad x, v \in \mathcal{H} .
$$

A direct calculation then shows that

$$
U_{x} U_{y}=e^{-i \operatorname{Im}\langle x, y\rangle} U_{x+y} \quad \text { for } \quad x, y \in \mathcal{H} .
$$


To obtain a unitary representation, we have to replace the additive group of $\mathcal{H}$ by the Heisenberg group

$$
\operatorname{Heis}(\mathcal{H}):=\mathbb{T} \times \mathcal{H} \quad \text { with } \quad(z, v)\left(z^{\prime}, v^{\prime}\right):=\left(z z^{\prime} e^{-i \operatorname{Im}\left\langle v, v^{\prime}\right\rangle}, v+v^{\prime}\right) .
$$

For this group, we obtain with (25) a unitary representation

$$
U: \operatorname{Heis}(\mathcal{H}) \rightarrow \mathrm{U}\left(\mathcal{F}_{+}(\mathcal{H})\right) \text { by } \quad U_{(z, v)}:=z U_{v} .
$$

In this physics literature, all this is expressed in terms of the so-called Weyl operators

$$
W(v):=U_{i v / \sqrt{2}}, \quad v \in \mathcal{H}
$$

satisfying the Weyl relations

$$
W(v) W(w)=e^{-i \operatorname{Im}\langle v, w\rangle / 2} W(v+w), \quad v, w \in \mathcal{H} .
$$

Definition 6.1. To each real subspace $V \subseteq \mathcal{H}$, we assign the von Neumann algebra $\mathcal{R}(V):=\mathcal{R}^{+}(V):=W(V)^{\prime \prime} \subseteq B\left(\mathcal{F}_{+}(\mathcal{H})\right)$ on the bosonic Fock space of $\mathcal{H}$.

LEMMA 6.2. We have

(i) $\mathcal{R}(\mathcal{H})=B\left(\mathcal{F}_{+}(\mathcal{H})\right)$, resp., the representation of $\operatorname{Heis}(\mathcal{H})$ on $\mathcal{F}_{+}(\mathcal{H})$ is irreducible.

(ii) $\mathcal{R}(V) \subseteq \mathcal{R}(W)^{\prime}$ if and only if $V \subseteq W^{\prime}$ (locality).

(iii) $\mathcal{R}(V)=\mathcal{R}(\bar{V})$.

(iv) $\Omega=\operatorname{Exp}(0) \in \mathcal{F}_{+}(\mathcal{H})$ is cyclic for $\mathcal{R}(V)$ if and only if $V+i V$ is dense in $\mathcal{H}$.

(v) $\Omega \in \mathcal{F}_{+}(\mathcal{H})$ is separating for $\mathcal{R}(V)$ if and only if $\bar{V} \cap i \bar{V}=\{0\}$.

(vi) $\Omega \in \operatorname{cs}(\mathcal{R}(V))$ if and only if $\bar{V}$ is standard.

Proof. (i) is well-known ( BR96, Prop. 5.2.4(3)]).

(ii) follows directly from the Weyl relations (26).

(iii) follows from the fact that $\mathcal{H} \rightarrow B\left(\mathcal{F}_{+}(\mathcal{H})\right), v \mapsto W_{v}$ is strongly continuous and $\mathcal{R}(V)$ is closed in the weak operator topology.

(iv) Assume that $\mathcal{K}:=\overline{V+i V} \neq \mathcal{H}$. Then $\mathcal{R}(V) \Omega \subseteq \mathcal{F}_{+}(\mathcal{K})$, so that $\Omega$ cannot be cyclic.

Suppose, conversely, that $\mathcal{K}=\mathcal{H}$ and that $f \in(\mathcal{R}(V) \Omega)^{\perp}$. Then the holomorphic function $\widehat{f}(v):=\langle f, \operatorname{Exp}(v)\rangle$ on $\mathcal{H}$ vanishes on $V$, hence also on $V+i V$, and since this subspace is dense in $\mathcal{H}$, we obtain $f=0$ because $\operatorname{Exp}(\mathcal{H})$ is total in $\mathcal{F}_{+}(\mathcal{H})$. We conclude that $\Omega$ is cyclic.

(v) In view of (iii), we may assume that $V$ is closed. Let $0 \neq w \in V \cap i V$. To see that $\Omega$ is not separating for $\mathcal{R}(V)$, it suffices to show that, for the one-dimensional Hilbert space $\mathcal{H}_{0}:=\mathbb{C} w$, the vector $\Omega$ is not separating for $\mathcal{R}(\mathbb{C} w)=B\left(\mathcal{F}_{+}(\mathbb{C} w)\right.$ ) (which follows from the irreducibility of the representation of $\operatorname{Heis}(\mathbb{C} w)$ on $\mathcal{F}_{+}(\mathbb{C} w)$ ). This is obviously the case because $\operatorname{dim} \mathcal{F}_{+}(\mathbb{C} w)>1$.

Suppose that $\mathcal{K}=\{0\}$. As $\mathcal{K}=V^{\prime \prime} \cap\left(i V^{\prime \prime}\right)=\left(V^{\prime}+i V^{\prime}\right)^{\prime}$, it follows that $V^{\prime}+i V^{\prime}$ is dense in $\mathcal{H}$. By (ii), $\Omega$ is cyclic for $\mathcal{R}\left(V^{\prime}\right)$ which commutes with $\mathcal{R}(V)$. Therefore $\Omega$ is separating for $\mathcal{R}(V)$.

(vi) follows from (iv) and (v).

REMARK 6.3. (a) $\mathcal{R}(V)$ is commutative if and only if $V \subseteq V^{\prime}$. For a standard subspace $V$ the relation $V^{\prime}=J V$ shows that this is equivalent to $V=V^{\prime}$, respectively to $\Delta=\mathbf{1}$ 
(Lemma 3.7).

(b) The imaginary part $\omega(\xi, \eta):=\operatorname{Im}\langle\xi, \eta\rangle$ turns $\mathcal{H}$ into a symplectic manifold $(\mathcal{H}, \omega)$. From this perspective, we may consider the algebras $\mathcal{R}(V)$ as "quantizations" of the algebra of measurable functions on the Lagrangian subspace $E:=\mathcal{H}^{J}$. If $V=V^{\prime}$, then $\mathcal{F}_{+}(\mathcal{H}) \cong L^{2}\left(E^{*}, \gamma\right)$, where $\gamma$ is a Gaussian probability measure on the algebraic dual space $E^{*}$ of $E$, endowed with the smallest $\sigma$-algebra for which all evaluation maps are measurable. Then the commutative von Neumann algebra $\mathcal{R}(V)$ is isomorphic to $L^{\infty}\left(E^{*}, \gamma\right)$. In general, if $V \nsubseteq V^{\prime}$, then $\mathcal{R}(V)$ is non-commutative and the degree of noncommutativity depends on the non-degeneracy of $\omega$ on $V$. It is "maximal" if $V \cap V^{\prime}=\{0\}$, which implies that $\mathcal{R}(V)$ is a factor by the following theorem.

TheOREm 6.4. ([Ar63, Thm. 1]) For closed real subspaces $V, W, V_{j}$ of $\mathcal{H}$, the following assertions hold:

(i) $\mathcal{R}(V) \subseteq \mathcal{R}(W)$ if and only if $V \subseteq W$ (isotony).

(ii) $R\left(\bigvee_{j \in J} V_{j}\right)=\bigvee_{j \in J} \mathcal{R}\left(V_{j}\right)$, where $\bigvee_{j \in J} V_{j}$ denotes the closed subspace generated by the $V_{j}$ and $\bigvee_{j \in J} \mathcal{R}\left(V_{j}\right)$ denotes the von Neumann algebra generated by the $\mathcal{R}\left(V_{j}\right)$.

(iii) $R\left(\bigcap_{j \in J} V_{j}\right)=\bigcap_{j \in J} \mathcal{R}\left(V_{j}\right)$.

(iv) $\mathcal{R}(V)^{\prime}=\mathcal{R}\left(V^{\prime}\right)$ (duality).

(v) $\mathcal{R}(V) \cap \mathcal{R}\left(V^{\prime}\right)=\mathcal{R}\left(V \cap V^{\prime}\right)$. In particular, the algebra $\mathcal{R}(V)$ is a factor if and only if $V \cap V^{\prime}=\{0\}$.

6.2. Fermionic Fock space. On the fermionic Fock space, the construction of the von Neumann algebras is slightly different but similar in spirit.

For $v_{1}, \ldots, v_{n} \in \mathcal{H}$, we define

$$
v_{1} \wedge \cdots \wedge v_{n}:=\frac{1}{\sqrt{n !}} \sum_{\sigma \in S_{n}} \operatorname{sgn}(\sigma) v_{\sigma(1)} \otimes \cdots \otimes v_{\sigma(n)},
$$

so that

$$
\left\langle v_{1} \wedge \cdots \wedge v_{n}, w_{1} \wedge \cdots \wedge w_{n}\right\rangle=\sum_{\sigma \in S_{n}} \operatorname{sgn}(\sigma)\left\langle v_{\sigma(1)}, w_{1}\right\rangle \cdots\left\langle v_{\sigma(n)}, w_{m}\right\rangle
$$

In $\mathcal{F}_{-}^{0}(\mathcal{H}) \cong \mathbb{C}$ we pick a unit vector $\Omega$, called the vacuum.

Definition 6.5. The $C A R$-algebra $\operatorname{CAR}(\mathcal{H})$ of $\mathcal{H}$ is a $C^{*}$-algebra, together with a continuous antilinear map $a: \mathcal{H} \rightarrow \operatorname{CAR}(\mathcal{H})$ satisfying the canonical anticommutation relations

$$
\left\{a(f), a(g)^{*}\right\}=\langle f, g\rangle \mathbf{1} \quad \text { and } \quad\{a(f), a(g)\}=0 \quad \text { for } \quad f, g \in \mathcal{H}
$$

and which has the universal property that, for any $C^{*}$-algebra $\mathcal{A}$ and any antilinear map $a^{\prime}: \mathcal{H} \rightarrow \mathcal{A}$ satisfying the above anticommutation relations, there exists a unique homomorphism $\varphi: \operatorname{CAR}(\mathcal{H}) \rightarrow \mathcal{A}$ with $\varphi \circ a=a^{\prime}$. This determines the pair $(\operatorname{CAR}(\mathcal{H}), a)$ up to isomorphism (BR96, Thm. 5.2.8]). We write $a^{*}(f):=a(f)^{*}$ and observe that this defines a complex linear map $a^{*}: \mathcal{H} \rightarrow \operatorname{CAR}(\mathcal{H})$.

REMARK 6.6. The $C^{*}$-algebra $\operatorname{CAR}(\mathcal{H})$ has an irreducible representation $\left(\pi_{0}, \mathcal{F}_{-}(\mathcal{H})\right)$ on the fermionic Fock space $\mathcal{F}_{-}(\mathcal{H})\left(\left[\right.\right.$ BR96, Prop. 5.2.2(3)]). The image $c(f):=\pi_{0}(a(f))$ 
acts by $c(f) \Omega=0$ and

$$
c(f)\left(f_{1} \wedge \cdots \wedge f_{n}\right)=\sum_{j=1}^{n}(-1)^{j-1}\left\langle f, f_{j}\right\rangle f_{1} \wedge \cdots \wedge f_{j-1} \wedge f_{j+1} \wedge \cdots \wedge f_{n} .
$$

Accordingly, we have

$$
c^{*}(f) \Omega=f \quad \text { and } \quad c^{*}(f)\left(f_{1} \wedge \cdots \wedge f_{n}\right)=f \wedge f_{1} \wedge \cdots \wedge f_{n} .
$$

Consider the hermitian operators

$$
b(f):=c(f)+c^{*}(f) \in \operatorname{CAR}(\mathcal{H})
$$

and note that

$$
\{b(f), b(g)\}=\left\{c(f), c^{*}(g)\right\}+\left\{c^{*}(f), c(g)\right\}=\langle f, g\rangle \mathbf{1}+\langle g, f\rangle \mathbf{1}=2 \beta(f, g) \mathbf{1},
$$

where

$$
\beta(f, g)=\operatorname{Re}\langle f, g\rangle \quad \text { for } \quad f, g \in \mathcal{H}
$$

is the real scalar product on $\mathcal{H}$.

Definition 6.7. Let $\mathcal{H}=\mathcal{H}_{\overline{0}} \oplus \mathcal{H}_{\overline{1}}$ be a 2-graded Hilbert space. Accordingly, $B(\mathcal{H})$ inherits a grading and therefore a Lie superbracket which on homogeneous elements is given by

$$
[A, B]_{\tau}:=A B-(-1)^{|A||B|} B A,
$$

where $|A|$ denotes the degree of a homogeneous element $A$. For a subset $E \subseteq B(\mathcal{H})$, we accordingly define the super-commutant by

$$
E^{\sharp}:=\left\{A \in B(\mathcal{H}):(\forall M \in E)[A, M]_{\tau}=0\right\} .
$$

For each homogeneous $M \in B(\mathcal{H})$, the operator $D_{M}(A):=[M, A]_{\tau}$ is a superderivation of the $\mathbb{Z}_{2}$-graded associative algebra $B(\mathcal{H})$ in the sense that

$$
D_{M}(A B)=D_{M}(A) B+(-1)^{|M||A|} A D_{M}(B) .
$$

It follows in particular that, if $E$ is spanned by homogeneous elements, then $E^{\sharp}$ is a von Neumann algebra adapted to the 2-grading of $B(\mathcal{H})$. Let $Z v=(-1)^{|v|} v(|v| \in\{0,1\})$ denote the parity operator on $\mathcal{H}$ and $\widetilde{Z} v=(-i)^{|v|} v$ (also known as the Klein twist $\left.\widetilde{Z}=\frac{1+i Z}{1+i \mathbf{1}}\right)$ which satisfies $\widetilde{Z}^{2}=Z$. For $A$ and $M$ odd we then have

$$
\left[\widetilde{Z}^{ \pm 1} A \widetilde{Z}^{\mp 1}, M\right]= \pm i Z\{A, M\}=-i Z[A, M]_{\tau} .
$$

This leads to

$$
E^{\sharp}=\widetilde{Z} E^{\prime} \widetilde{Z}^{-1}=\widetilde{Z}^{-1} E^{\prime} \widetilde{Z}
$$

for any graded subspace $E \subseteq B(\mathcal{H})$.

As in [Fo83, we associated to every real linear subspace $V \subseteq \mathcal{H}$ a von Neumann subalgebra

$$
\mathcal{R}(V):=\mathcal{R}^{-}(V):=b(V)^{\prime \prime} \subseteq B\left(\mathcal{F}_{-}(\mathcal{H})\right) .
$$

We list some properties of this assignment (cf. [Fo83, Prop. 2.5] for (iv) and (v)):

LEMMA 6.8. We have

(i) $\mathcal{R}(\mathcal{H})=B\left(\mathcal{F}_{-}(\mathcal{H})\right)$, resp., the representation of $\mathrm{CAR}(\mathcal{H})$ on $\mathcal{F}_{-}(\mathcal{H})$ is irreducible. 
(ii) $\mathcal{R}(V)=\mathcal{R}(\bar{V})$.

(iii) $\mathcal{R}(V)$ and $\mathcal{R}(W)$ super-commute if and only if $V \perp_{\beta} W$ (twisted duality).

(iv) The vacuum $\Omega$ is cyclic for $\mathcal{R}(V)$ if and only if $V+i V$ is dense in $\mathcal{H}$.

(v) The vacuum $\Omega$ is separating for $\mathcal{R}(V)$ if and only if $\bar{V} \cap i \bar{V}=\{0\}$.

(vi) $\Omega \in \operatorname{cs}(\mathcal{R}(V))$ if and only if $\bar{V}$ is standard.

Proof. (i) is well-known ([BR96, Prop. 5.2.2(3)]).

(ii) follows from the fact that $b: \mathcal{H} \rightarrow B\left(\mathcal{F}_{-}(\mathcal{H})\right)$ is continuous.

(iii) follows immediately from (30).

(iv) We explain how this can be derived from [BJL02, Prop. 3.4], where a different setting is used: Consider a conjugation $\Gamma$ on a complex Hilbert space $\mathcal{K}$ and a corresponding basis projection $P$, i.e., $\Gamma P \Gamma=\mathbf{1}-P$. For $v \in \mathcal{K}^{\Gamma}$ we then have the orthogonal decomposition $v=P v+(\mathbf{1}-P) v$, where both summands are exchanged by $\Gamma$, hence have the same length. Therefore the map

$$
\Phi: \mathcal{K}^{\Gamma} \rightarrow P \mathcal{K}, \quad \Phi(v)=\sqrt{2} P v
$$

is an isometry between the real Hilbert space $\mathcal{K}^{\Gamma}$ and the complex Hilbert space $\mathcal{H}:=P \mathcal{K}$. The antilinear map

$$
a: \mathcal{K} \rightarrow \operatorname{CAR}(\mathcal{H}), \quad a(f):=c^{*}(P \Gamma f)+c(P f)
$$

then satisfies

$$
a(\Gamma f)=a(f)^{*} \quad \text { for } \quad f \in \mathcal{K}
$$

and $a$ is the unique antilinear extension of the map $\left.a\right|_{\mathcal{K}^{\Gamma}}=b \circ P: \mathcal{K}^{\Gamma} \rightarrow \operatorname{CAR}(\mathcal{H})$.

For any $\Gamma$-invariant subspace $\mathcal{V} \subseteq \mathcal{K}$, we therefore have

$$
a(\mathcal{V})=b\left(P \mathcal{V}^{\Gamma}\right)_{\mathbb{C}}=b\left(\Phi\left(\mathcal{V}^{\Gamma}\right)\right)_{\mathbb{C}}
$$

and thus, for the real subspace $V:=\Phi\left(\mathcal{V}^{\Gamma}\right)=P\left(\mathcal{V}^{\Gamma}\right) \subseteq \mathcal{H}$,

$$
a\left(\mathcal{V}^{\Gamma}\right)^{\prime \prime}=a(\mathcal{V})^{\prime \prime}=b\left(\Phi\left(\mathcal{V}^{\Gamma}\right)\right)^{\prime \prime}=\mathcal{R}(V)^{\prime \prime} .
$$

As $V+i V=P\left(\mathcal{V}_{\mathbb{C}}^{\Gamma}\right)=P(\mathcal{V})$, BJL02, Prop. 3.4] implies that $P(\mathcal{V})$ is dense in $P(\mathcal{H})$ if and only if $\Omega$ is $\mathcal{R}(V)$-cyclic, and (iv) follows.

(v) In view of (ii), we may assume that $V$ is closed. Let $0 \neq w \in W:=V \cap i V$. To see that $\Omega$ is not separating for $\mathcal{R}(V)$, it suffices to show that, for the one-dimensional Hilbert space $\mathcal{H}_{0}:=\mathbb{C} w$, the vector $\Omega$ is not separating for $\mathcal{R}(\mathbb{C} w)=B\left(\mathcal{F}_{-}(\mathbb{C} w)\right)$. This follows from the irreducibility of the representation of $\operatorname{CAR}(\mathbb{C} w) \cong M_{2}(\mathbb{C})$ on $\mathcal{F}_{-}(\mathbb{C} w) \cong \mathbb{C}^{2}$ which has no separating vector (see (i)).

Suppose, conversely, that $W=\{0\}$. As $W=\left(V^{\perp}+i V^{\perp}\right)^{\perp}$, the subspace $V^{\perp}+i V^{\perp}$ is dense in $\mathcal{H}$. By (iii), $\Omega$ is cyclic for $\mathcal{R}\left(V^{\perp}\right)$ which anticommutes with $\mathcal{R}(V)$. Therefore $\Omega$ is separating for $\mathcal{R}(V)$.

The following theorem is the fermionic version of the duality result in Theorem 6.4(iii) ([BJL02, Thm. 7.1], [Fo83, Thm. 2.4(v)]).

Theorem 6.9 (Fermionic Duality Theorem).

$$
\mathcal{R}\left(V^{\perp_{\beta}}\right)=\mathcal{R}(V)^{\sharp}=\left\{A \in B\left(\mathcal{F}_{-}(\mathcal{H})\right):(\forall v \in V)[A, b(v)]_{\tau}=0\right\}=\widetilde{Z}^{-1} \mathcal{R}(V)^{\prime} \widetilde{Z}
$$

for every real linear subspace $V \subseteq \mathcal{H}$. 
To match our notation with Foit's in Fo83, we note that Foit's operator $V:=\frac{1}{\sqrt{2}}(\mathbf{1}-i Z)$ satisfies $V=e^{-\pi i / 4} \widetilde{Z}^{-1}$, so that $\widetilde{Z}^{-1} A \widetilde{Z}=V A V^{*}$ for every operator $A$ on $\mathcal{F}_{-}(\mathcal{H})$.

6.3. From antiunitary representations to local nets. For a closed real subspace $V$ of the Hilbert space $\mathcal{H}$, we write $\mathcal{R}^{ \pm}(V) \subseteq B\left(\mathcal{F}_{ \pm}(\mathcal{H})\right)$ for the associated von Neumann algebras on the bosonic and fermionic Fock space.

Proposition 6.10. For a closed real subspace $V \subseteq \mathcal{H}$, the vacuum $\Omega$ is cyclic and separating for the von Neumann algebras $\mathcal{R}^{ \pm}(V) \subseteq B\left(\mathcal{F}_{ \pm}(\mathcal{H})\right)$ if and only if $V$ is a standard subspace of $\mathcal{H}$. The corresponding modular objects $\left(\Delta_{V}^{ \pm}, J_{V}^{ \pm}\right)$on $\mathcal{F}_{ \pm}(\mathcal{H})$ are obtained by second quantization from the modular objects $\left(\Delta_{V}, J_{V}\right)$ associated to $V$, in the sense that

$$
\Delta_{V}^{ \pm}=\Gamma_{ \pm}\left(\Delta_{V}\right), \quad J_{V}^{+}=\Gamma_{+}\left(J_{V}\right) \quad \text { and } \quad J_{V}^{-}=\widetilde{Z} \Gamma_{-}\left(i J_{V}\right)
$$

Proof. The first assertion follows from Lemmas 6.2 and 6.8. For the identification of the modular objects, we refer to [FG89, Thm. 1.4] (see also [EO73]) in the bosonic case and to [Fo83, Prop. 2.8] for the fermionic case (see also [BJL02, Cor. 5.4], [Lle09, Thm. 4.13]).

Remark 6.11. (a) The twists arising in Theorem 6.9 and Proposition 6.10 arise from the fact that the fermionic situation has to take the 2-grading on $\mathcal{F}_{-}(\mathcal{H})$ into account. In particular Theorem 6.9 takes its most natural form $\mathcal{R}\left(V^{\perp_{\beta}}\right)=\mathcal{R}(V)^{\sharp}$ if the commutant is defined in terms of the super bracket.

(b) If $\mathcal{M}$ is a $\mathbb{Z}_{2}$-graded von Neumann algebra on the $\mathbb{Z}_{2}$-graded Hilbert space $\mathcal{H}=$ $\mathcal{H}_{0} \oplus \mathcal{H}_{1}$ and $\Omega \in \mathcal{H}_{0}$ is a cyclic separating vector, then the theory of Lie superalgebras suggests to consider the antilinear involution $\left(x_{0}+x_{1}\right)^{\sharp}:=x_{0}^{*}-i x_{1}^{*}$ instead of the operator adjoint. Then the corresponding unitary Lie superalgebra is

$$
\mathfrak{u}(\mathcal{M})=\left\{x \in \mathcal{M}: x^{\sharp}=-x\right\}=\left\{x=x_{0}+x_{1} \in \mathcal{M}: x_{0}^{*}=-x_{0}, x_{1}^{*}=-i x_{1}\right\} .
$$

Accordingly, modular theory can be based on the unbounded antilinear operator defined by $\widetilde{S}(M \Omega):=M^{\sharp} \Omega=\widetilde{Z} S(M \Omega)$ for $M \in \mathcal{M}$. The polar decomposition $\overline{\widetilde{S}}=\widetilde{J} \Delta^{1 / 2}$ results in the pair $(\widetilde{J}, \Delta)$ of modular objects, where $\Delta$ is unchanged, but $\widetilde{J}=\widetilde{Z} J$. This leads to the relation

$$
\widetilde{J} \mathcal{M} \widetilde{J} \mathcal{M}^{\prime} \widetilde{Z}^{-1}=\mathcal{M}^{\sharp},
$$

which is a super version of $J \mathcal{M} J=\mathcal{M}^{\prime}$.

We also obtain with (35)

$$
\widetilde{J_{V}^{-}}=\widetilde{Z}^{2} \Gamma_{-}\left(i J_{V}\right)=Z \Gamma_{-}\left(i J_{V}\right)=\Gamma_{-}\left(-i J_{V}\right) .
$$

To obtain a situation where the modular objects on $\mathcal{F}^{-}(\mathcal{H})$ are simply given by second quantization, one may consider the von Neumann algebras $\widetilde{\mathcal{R}}^{-}(V):=\mathcal{R}^{-}(\zeta V)$ for $\zeta:=$ $e^{\pi i / 4}$ instead. The standard subspace $\widetilde{V}:=\zeta V$ satisfies $\Delta_{\widetilde{V}}=\Delta_{V}$ and $J_{\widetilde{V}}=i J_{V}$, so that the modular conjugation corresponding to $\widetilde{\mathcal{R}}^{-}(V)$ is

$$
\widetilde{J_{\zeta V}^{-}}=\Gamma_{-}\left(-i J_{\zeta V}\right)=\Gamma_{-}\left(J_{V}\right)
$$

REMARK 6.12. Let $(U, \mathcal{H})$ be an antiunitary representation of $\left(G, G_{1}\right)$ on $\mathcal{H}$ and $\gamma: \mathbb{R}^{\times} \rightarrow G$ be a homomorphism with $\gamma(-1) \notin G_{1}$, so that it specifies a standard 
subspace $V_{\gamma} \subseteq \mathcal{H}$ (Proposition [5.6). Consider the antiunitary representation

$$
\Gamma_{ \pm}: \mathrm{AU}(\mathcal{H}) \rightarrow \operatorname{AU}\left(\mathcal{F}_{ \pm}(\mathcal{H})\right)
$$

of the antiunitary group of $\mathcal{H}$ on the corresponding Fock spaces. Then $\Gamma_{ \pm} \circ U$ is an antiunitary representation of $\left(G, G_{1}\right)$ on $\mathcal{F}_{ \pm}(\mathcal{H})$, so that we also obtain standard subspaces $V_{\gamma}^{ \pm} \subseteq \mathcal{F}_{ \pm}(\mathcal{H})$. The pair $\left(\mathcal{R}^{+}\left(V_{\gamma}\right), \Omega\right)$ then satisfies

$$
V_{\gamma}^{+}=\overline{\mathcal{R}^{+}\left(V_{\gamma}\right)_{h} \Omega}
$$

and in the fermionic case the pair $\left(\mathcal{R}^{-}\left(V_{\gamma}\right), \Omega\right)$ leads to the correct modular operator $\Delta_{V_{\gamma}}^{-}$, but to the modular conjugation $\widetilde{Z} \Gamma_{-}\left(i J_{V_{\gamma}}\right)$.

7. Perspectives. For a detailed exposition of the results mentioned below, we refer to the forthcoming paper NÓ17.

7.1. The Virasoro group. In $\operatorname{Diff}\left(\mathbb{S}^{1}\right)$ we consider the involution on $\mathbb{S}^{1} \cong \mathbb{T} \subseteq \mathbb{C}$, given by $r(z)=\bar{z}$. We consider the group $G:=\operatorname{Diff}\left(\mathbb{S}^{1}\right) \cong \operatorname{Diff}\left(\mathbb{S}^{1}\right)_{0} \rtimes\{\mathbf{1}, r\}$. One can show that all projective unitary positive energy representations of Diff $\left(\mathbb{S}_{1}\right)_{0}$ extend naturally to projective antiunitary representations of $G$. To obtain antiunitary representations, one has to replace $G$ by a central extension Vir $\rtimes\{\mathbf{1}, r\}$, where Vir is the simply connected Virasoro group.

Another closely related "infinite dimensional" group that occurs in the context of modular localization is the free product $\mathrm{PSL}_{2}(\mathbb{R}) *_{\mathrm{Aff}(\mathbb{R})_{0}} \mathrm{PSL}_{2}(\mathbb{R})$ of two copies of $\mathrm{PSL}_{2}(\mathbb{R})$ over the connected affine group (GLW98]).

7.2. Euclidean Jordan algebras. Minkowski spaces are particular examples of simple euclidean Jordan algebras, namely those of rank 2 (cf. [FK94). Many of the geometric structures of Minkowski spaces and their conformal completions are also available for general simple euclidean Jordan algebras, where the role of the lightcone $V_{+}$is played by the open cone of invertible squares. There also exists a natural causal compactification $\widehat{V}$ which carries a causal structure. The corresponding conformal group $G:=\operatorname{Conf}(V)$ has an index 2 subgroup $G_{1}$ preserving the causal structure on $\widehat{V}$; other group elements reverse it. In $\widehat{V}$, the set $\mathcal{W}^{c}:=\left\{g V_{+}: g \in \operatorname{Conf}(V)\right\}$ specializes for Minkowski spaces to the set of conformal wedge domains, which include in particular the light cone and double cones (cf. Example [5.14). Moreover, the homomorphism

$$
\gamma_{V_{+}}: \mathbb{R}^{\times} \rightarrow \mathrm{GL}(V), \quad \gamma(t) v=t v
$$

is naturally specified because $\gamma_{V_{+}}\left(\mathbb{R}_{+}^{\times}\right)$is central in the identity component of the stabilizer $G_{V_{+}}$. Therefore any antiunitary positive energy representation of $G$ yields a net of standard subspaces indexed by $\mathcal{W}^{c}$. In [NÓ17] we obtain a classification of these representations along the lines of $₫ 2.2$. The subsemigroups $S_{V_{+}}:=\left\{g \in G: g V_{+} \subseteq V_{+}\right\} \subseteq G$ also leads to a natural generalization of Borchers pairs in this context.

7.3. Hermitian groups. The conformal group $\operatorname{Conf}(V)$ of a euclidean Jordan algebra can be identified with the group AAut $\left(T_{V_{+}}\right)$of holomorphic and antiholomorphic automorphisms of the corresponding tube domain $T_{V_{+}}=V_{+}+i V$. This suggests that some of the crucial structure relevant for antiunitary representations can still be obtained for 
the groups $G:=\operatorname{AAut}(\mathcal{D})$ of all holomorphic and antiholomorphic automorphisms of a bounded symmetric domain $\mathcal{D}$. The irreducible antiunitary positive energy representations can also be parametrized in a natural way by writing $G=G_{1} \rtimes\{\mathrm{id}, \sigma\}$, where $\sigma$ is an antiholomorphic involution of $\mathcal{D}$ (NÓ17]). Here there are many homomorphisms $\gamma:\left(\mathbb{R}^{\times}, \mathbb{R}_{+}^{\times}\right) \rightarrow\left(G, G_{1}\right)$ with $\gamma(-1)=\sigma$, but one cannot expect $\gamma\left(\mathbb{R}_{+}^{\times}\right)$to be central in $G^{\sigma}$, which can be achieved for tube type domains (coming from euclidean Jordan algebras).

7.4. Analytic extension. We have seen that, for antiunitary representations of $\operatorname{Aff}(\mathbb{R})$, the positive energy condition appears quite naturally from the order structure on the set of standard subspaces. If $(U, \mathcal{H})$ is an antiunitary representation of $G$ containing copies of Aff $(\mathbb{R})$ coming from half-sided modular inclusions, it follows that the closed convex cone

$$
C_{U}:=\{x \in \mathfrak{g}:-i \mathrm{~d} U(x) \geq 0\}
$$

is non-trivial. This further leads to an analytic extension of the representation to the domain $G \exp \left(i C_{U}\right)$ (see $[\mathrm{Ne} 00$ for details on this process).

On the other hand, antiunitary representations of $\mathbb{R}^{\times}$correspond to modular objects $(\Delta, J)$ and the orbit maps of elements $v \in V$ extend to the strip $\{z \in \mathbb{C}: 0 \leq \operatorname{Im} z \leq \pi\}$ (Remark 3.31). Composing families of homomorphisms $\gamma: \mathbb{R}^{\times} \rightarrow G$ with an antiunitary representation, we therefore expect analytic continuation of $U$ to natural complex domains containing $G$ in their boundary.

It would be very interesting to combine these two types of analytic continuations in a uniform manner, in the same spirit as the KMS condition is a generalization of the ground state condition (corresponding to positive energy) ([BR96]). One may further expect that this leads to "euclidean realizations" of antiunitary representations of $G$ by unitary representations of a Cartan dual group in the sense of the theory of reflection positivity developed in in NÓ14, NÓ16; see also Sch06 for relations with modular theory. Maybe it can even be combined with the analytic extensions to the crown of a Riemannian symmetric space ([KS05]).

7.5. Geometric standard subspaces. In QFT, the algebras $\mathcal{R}(V)$ are supposed to correspond to regions in some spacetime $M$. Therefore one looks for standard subspaces $V(\mathcal{O})$ that are naturally associated to a domain in some spacetime, such as Hardy spaces (Example 3.16) or the standard subspaces $K(\mathcal{O})$ constructed in [FG89] for free fields. From the perspective of antiunitary group representations, a natural class of representations of a pair $\left(G, G_{1}\right)$ are those realized in spaces $\mathcal{H}_{D} \subseteq C^{-\infty}(M)$ of distributions on a manifold $M$ on which $G_{1}$ acts. Here $\mathcal{H}_{D}$ is the Hilbert space completion of the space $C_{c}^{\infty}(M)$ of test functions with respect to the scalar product given by a positive definite distribution $D$ on $M \times M$ via

$$
\langle\xi, \eta\rangle_{D}=\int_{M \times M} \overline{\xi(x)} \eta(y) d D(x, y)
$$

(cf. NÓ14). We associate to each open subset $\mathcal{O} \subseteq M$ a subset $\mathcal{H}_{D}(\mathcal{O})$ generated by the space $C_{c}^{\infty}(\mathcal{O})$ of test functions supported in $\mathcal{O}$. In this context it is an interesting problem to find natural antiunitary extensions of the representation of $G_{1}$ to $G$ such that 
some of the corresponding standard subspaces (Proposition 3.2) have natural geometric descriptions. In this context the detailed analysis of KMS conditions for unitary representations of $\mathbb{R}$ in [NÓ16] should be a crucial tool because one typically expects standard subspaces to be described in terms of analytic continuations of distributions on some domain $\mathcal{O} \subseteq M$ to a complex manifold containing $\mathcal{O}$, which links this problem to 77.4 (cf. NÓ17b]). As one also wants the modular unitaries to act geometrically on the manifold $M$, the case $G_{1}=\mathbb{R}$ acting by translations on $\mathbb{R}$ considered in [NÓ16] is of key importance.

Conversely, one may also consider Hilbert spaces $\mathcal{H}$ of holomorphic functions on a complex manifold $M$ on which $G$ acts in such a way that $G_{1}$ acts by holomorphic maps and $G \backslash G_{1}$ by antiholomorphic ones. Then any $\gamma \in \operatorname{Hom}\left(\left(\mathbb{R}^{\times}, \mathbb{R}_{+}^{\times}\right),\left(G, G_{1}\right)\right)$ leads to a standard subspace of $\mathcal{H}$. Many natural examples of this type arise from $\S \S 7.2$ and 7.3 . In particular, the representation of $\operatorname{AU}(\mathcal{H})$ on $\mathcal{F}_{+}(\mathcal{H})$ is of this type if we identify $\mathcal{F}_{+}(\mathcal{H})$ with the Hilbert space of holomorphic functions on $\mathcal{H}$ with the reproducing kernel $K(\xi, \eta)=$ $e^{\langle\xi, \eta\rangle}$ (cf. [Ne0]).

7.6. Dual pairs in the Heisenberg group. Let $\mathcal{H}$ be a complex Hilbert space and $V \subseteq \mathcal{H}$ be a real linear subspace. We consider the corresponding subgroup $\operatorname{Heis}(V):=$ $\mathbb{T} \times V \subseteq \operatorname{Heis}(\mathcal{H})(6.1)$. The centralizer of this subgroup in $\operatorname{Heis}(\mathcal{H})$ coincides with Heis $\left(V^{\prime}\right)$. If $V$ is closed, we thus obtain a dual pair (Heis $(V)$, Heis $\left.\left(V^{\prime}\right)\right)$ of subgroups in $\operatorname{Heis}(\mathcal{H})$ in the sense that both subgroups are their mutual centralizers.

Remark 7.1. For a closed linear subspace $V \subseteq \mathcal{H}$, we have $\operatorname{Herm}(\mathcal{H})=\overline{\operatorname{Heis}(V) \operatorname{Heis}\left(V^{\prime}\right)}$ if and only if $V+V^{\prime}$ is dense in $\mathcal{H}$, which is equivalent to $\left(V+V^{\prime}\right)^{\prime}=V \cap V^{\prime}=\{0\}$ (cf. Lemma 3.7). If this is the case, then $\mathcal{R}(V) \subseteq B\left(\mathcal{F}_{+}(\mathcal{H})\right)$ is a factor by Theorem6.4. Accordingly, the restriction of the irreducible Fock representation $\left(U, \mathcal{F}_{+}(\mathcal{H})\right)$ of $\operatorname{Heis}(\mathcal{H})$ to $\operatorname{Heis}(V)$ is a factor representation and the same holds for Heis $\left(V^{\prime}\right)$. We thus obtain many interesting types of factor representations of Heisenberg groups of the type Heis $(V)$ simply by restricting an irreducible representation of Heis $(\mathcal{H})$. In vD71] this approach is used to realize quasi-free representations of $\operatorname{Heis}(V)$ in a natural way.

Remark 7.2. (a) Suppose that $G$ is a group which is the product $G=G_{1} G_{2}$ of two subgroups $G_{1}$ and $G_{2}$ such that $G_{1}=Z_{G}\left(G_{2}\right)$ and $G_{2}=Z_{G}\left(G_{1}\right)$. Then $G_{1} \cap G_{2}=Z(G)$ and every unitary representation $(U, \mathcal{H})$ of $G$ restricts to factor representations of the subgroups $G_{j}$.

(b) A typical example arises from a von Neumann algebra $\mathcal{M} \subseteq B(\mathcal{H})$ in symmetric form, i.e., there exists a conjugation $J$ with $J \mathcal{M} J=\mathcal{M}^{\prime}$ (Definition 4.4). Then $G:=$ $\mathrm{U}(\mathcal{M}) \mathrm{U}\left(\mathcal{M}^{\prime}\right)$ is a product of two subgroups $G_{1}:=\mathrm{U}(\mathcal{M})$ and $G_{2}:=\mathrm{U}\left(\mathcal{M}^{\prime}\right)$ satisfying this condition. The representation of $G$ on $\mathcal{H}$ is multiplicity free because $G^{\prime}=\mathcal{M} \cap \mathcal{M}^{\prime}$ is the center of $\mathcal{M}$, hence abelian. It is irreducible if and only if $\mathcal{M}$ is a factor, and then the representations of the subgroups $G_{1}$ and $G_{2}$ are factor representations. Note that the representation of $G$ extends to an antiunitary representation of $G \rtimes\{\mathbf{1}, j\}$, where $j(g)=J g J$.

REMARK 7.3. Similar structures also arise for infinite dimensional Lie groups such as $\operatorname{Diff}\left(\mathbb{S}^{1}\right)$, (doubly extended) loop groups and oscillator groups because modular objects 
provide information on restrictions of irreducible representations to factorial representations of subgroups (cf. Wa98 for loop groups). So one should also try to develop the theory of modular localization for antiunitary representations of infinite dimensional Lie groups.

7.7. A representation theoretic perspective on modular localization. The analysis of ordered families of von Neumann algebras with a common cyclic separating vector carried out by Borchers in Bo97. should also have a natural counterpart in the context of standard subspaces, in the spirit of the translation mechanism described in Subsection 4.2. It would be interesting to see if the corresponding results can be formulated entirely in group theoretic terms, concerning multiplicative one-parameter groups of some pair $\left(G, G_{1}\right)$ (cf. Proposition 5.6). As we have seen in $\S \$ 3.4$ and 3.5 , this works perfectly well for half-sided modular inclusions and modular intersections,

The same could be said about Wiesbrock's program, concerning the generation of Haag-Kastler nets from finite configurations of von Neumann algebras with common cyclic separating vectors ([Wi93c, Wi97b, Wi98, KW01]).

A. Appendices. In this short appendix we collect some general lemmas used in the main text.

\section{A.1. A lemma on von Neumann algebras.}

Lemma A.1. Let $\mathcal{M} \subseteq \mathcal{H}$ be a von Neumann algebra, $\alpha: \mathcal{M} \rightarrow \mathcal{M}$ a real-linear weakly continuous automorphism and $U \in \mathrm{U}(\mathcal{M})$ be a unitary element. Then the following assertions hold:

(a) If $\alpha$ is complex linear and $\alpha(U)=U$, then there exists a $V \in \mathrm{U}(\mathcal{M})$ with $\alpha(V)=V$ and $V^{2}=U$.

(b) If $\alpha$ is complex linear and $\alpha(U)=U^{-1}$ with $\operatorname{ker}(U+\mathbf{1})=\{0\}$, then there exists a $V \in \mathrm{U}(\mathcal{M})$ with $\alpha(V)=V^{-1}$ and $V^{2}=U$.

(c) If $\alpha$ is antilinear and $\alpha(U)=U^{-1}$, then there exists a $V \in \mathrm{U}(\mathcal{M})$ with $\alpha(V)=V^{-1}$ and $V^{2}=U$.

(d) If $\alpha$ is antilinear and $\alpha(U)=U$ with $\operatorname{ker}(U+\mathbf{1})=\{0\}$, then there exists a $V \in$ $\mathrm{U}(\mathcal{M})$ with $\alpha(V)=V$ and $V^{2}=U$.

Proof. (a) Let $P$ be the spectral measure of $U$ on the circle $\mathbb{T} \subseteq \mathbb{C}$ with $U=\int_{\mathbb{T}} z d P(z)$. As $P$ is uniquely determined by $U$ and $\alpha$ is complex linear, we obtain $\alpha(P(E))=P(E)$ for every measurable subset $E \subseteq \mathbb{T}$. For every measurable function $S: \mathbb{T} \rightarrow \mathbb{T}$ with $S(z)^{2}=z$ for $z \in \mathbb{T}$, we obtain by $V:=\int_{\mathbb{T}} S(z) d P(z)$ a square root of $U$ fixed by $\alpha$.

(b) Now our assumptions implies that $P(\{-1\})=0$, so that we find a unique spectral measure $\widetilde{P}$ on the open interval $(-\pi, \pi)$ with $U=\int_{-\pi}^{\pi} e^{i \theta} d \widetilde{P}(\theta)$. From $\alpha(U)=U^{-1}$ we derive $\alpha(\widetilde{P}(E))=\widetilde{P}(-E)$ for every measurable subset $E \subseteq(-\pi, \pi)$. Therefore $V:=$ $\int_{-\pi}^{\pi} e^{i \theta / 2} d P(\theta)$ is a square root of $U$ satisfying $\alpha(V)=V^{-1}$.

(c) We consider the von Neumann algebra $\mathcal{N} \subseteq \mathcal{M}$ generated by $U$. Then $\mathcal{N}$ is abelian and $\alpha$-invariant. Therefore $\beta(N):=\alpha\left(N^{*}\right)$ defines a complex linear automorphism of $\mathcal{N}$. It satisfies $\beta(U)=U$, so that the existence of $V$ follows from (a).

(d) We argue as in (c), but now (b) applies. 
ExAmple A.2. For $\mathcal{M}=\mathbb{C}, \alpha=\mathrm{id}_{\mathbb{C}}$ and $U=\mathbf{- 1}$ there exists no unitary $V \in \mathrm{U}(\mathcal{M})$ with $V^{2}=U$ and $V=\alpha(V)=V^{-1}$. This shows that the extra assumption in (b) is really needed.

\section{A.2. Two lemmas on groups.}

LemmA A.3. Let $G$ be a group and $G^{\sharp}:=(G \times G) \rtimes\{\mathbf{1}, \tau\}$, where $\tau(g, h)=(h, g)$ is the flip automorphism. We consider the subset $D:=\left\{\left(g, g^{-1}\right): g \in G\right\}$. Then the following assertions hold:

(a) $\operatorname{grp}(D)=\left(G^{\prime} \times\{e\}\right) D$, where $G^{\prime} \subseteq G$ is the commutator subgroup.

(b) The conjugacy class $C_{(e, e, \tau)}$ of the involution $(e, e, \tau) \in G^{\sharp}$ coincides with $D \times\{\tau\}$ and

$$
\operatorname{grp}\left(C_{(e, \tau)}\right)=\left(\left(G^{\prime} \times\{e\}\right) D\right) \times\{\mathbf{1}, \tau\} .
$$

Proof. (a) For $g, h \in G$, the relation

$$
\left(g, g^{-1}\right)\left(h, h^{-1}\right)=\left(g h, g^{-1} h^{-1}\right)=\left(g h g^{-1} h^{-1}, e\right)\left(h g,(h g)^{-1}\right)
$$

implies that $G^{\prime} \times\{e\} \subseteq \operatorname{grp}(D)$. Conversely, it is easy to see that $\left(G^{\prime} \times\{e\}\right) D$ is a subgroup of $G \times G$.

(b) The first assertion follows from $(g, h, \mathbf{1})(e, e, \tau)(g, h, \mathbf{1})^{-1}=\left(g h^{-1}, h g^{-1}, \tau\right)$. The second assertion follows from (a).

LemmA A.4. Let $G_{1} \subseteq G$ be a subgroup of index two, $r \in G \backslash G_{1}$, and $\varphi: G_{1} \rightarrow H$ be a group homomorphism. If $h \in H$ satisfies $h^{2}=\varphi\left(r^{2}\right)$ and $h \varphi(g) h^{-1}=\varphi\left(r g r^{-1}\right)$ for $g \in G_{1}$, then $\widehat{\varphi}(g r):=\varphi(g) h$ and $\widehat{\varphi}(g):=\varphi(g)$ for $g \in G_{1}$ defines an extension of $\varphi$ to a homomorphism $\widehat{\varphi}: G \rightarrow H$.

Proof. First we observe that $\widehat{\varphi}(g u)=\widehat{\varphi}(g) \widehat{\varphi}(u)$ obviously holds for $g \in G_{1}$ and $u \in G$. For $g \in G_{1}$ we further have

$$
\widehat{\varphi}(r g)=\widehat{\varphi}\left(r g r^{-1} r\right)=\varphi\left(r g r^{-1}\right) h=h \varphi(g) h^{-1} h=h \varphi(g)=\widehat{\varphi}(r) \widehat{\varphi}(g) .
$$

Finally, we note that, for $g \in G_{1}$,

$$
\widehat{\varphi}(r g r)=\widehat{\varphi}\left(r g r^{-1} r^{2}\right)=\varphi\left(r g r^{-1}\right) \varphi\left(r^{2}\right)=h \varphi(g) h^{-1} \varphi\left(r^{2}\right)=h \varphi(g) h=\widehat{\varphi}(r) \widehat{\varphi}(g r) .
$$

This implies that $\widehat{\varphi}$ is a group homomorphism.

\section{A.3. Symmetric spaces.}

Definition A.5. (a) Let $M$ be a set and

$$
\mu: M \times M \rightarrow M, \quad(x, y) \mapsto x \cdot y=: s_{x}(y)
$$

be a map with the following properties:

(S1) $x \cdot x=x$ for all $x \in M$, i.e., $s_{x}(x)=x$.

(S2) $x \cdot(x \cdot y)=y$ for all $x, y \in M$, i.e., $s_{x}^{2}=\operatorname{id}_{M}$.

(S3) $s_{x}(y \cdot z)=s_{x}(y) \cdot s_{x}(z)$ for all $x, y \in M$, i.e., $s_{x} \in \operatorname{Aut}(M, \mu)$.

Then we call $(M, \mu)$ a reflection space.

(b) If $M$ be a smooth manifold and $\mu: M \times M \rightarrow M$ is a smooth map turning $(M, \mu)$ into a reflection space, then it is called a symmetric space (in the sense of Loos) if, in addition, each $x$ is an isolated fixed point of $s_{x}$ ([Lo69]). 
ExAmple A.6. (a) Any group $G$ is a reflection space with respect to the product

$$
g \bullet h:=s_{g}(h):=g h^{-1} g .
$$

The subset $\operatorname{Inv}(G)$ of involutions in $G$ is a reflection subspace on which the product takes the form $s_{g}(h):=g h g=g h g^{-1}$. More generally, the subset

$$
G_{2}:=\left\{g \in G: g^{2} \in Z(G)\right\}
$$

is a reflection subspace of $G$ because $g^{2}, h^{2} \in Z(G)$ implies

$$
\left(g h^{-1} g\right)^{2}=g h^{-1} g^{2} h^{-1} g=g^{2} h^{-2} g^{2} \in Z(G) .
$$

This calculation even shows that the square map $G_{2} \rightarrow Z(G), g \mapsto g^{2}$ is a homomorphism of reflection spaces.

(b) Suppose that $G$ is a Lie group and $\tau \in \operatorname{Aut}(G)$ an involution. For any open subgroup $H \subseteq G^{\tau}=\operatorname{Fix}(\tau)$, we obtain on the coset space $M=G / H$ the structure of a symmetric space by

$$
x H \bullet y H:=x \tau(x)^{-1} \tau(y) H .
$$

Acknowledgments. K.-H. Neeb acknowledges supported by DFG-grant NE 413/9-1. The research of G. Ólafsson was supported by NSF grant DMS-1101337. Both authors thank Arthur Jaffe for supporting a visit at Harvard University, where some of this work was done.

\section{References}

[Ar63] Araki, H., A lattice of von Neumann algebras associated with the quantum theory of a free Bose field, J. Math. Phys. 4 (1963), 1343-1362

[Ar64] - von Neumann algebras of local observables for free scalar field, J. Mathematical Phys. 5 (1964), 1-13

[Ar99] - "Mathematical Theory of Quantum Fields," Int. Series of Monographs on Physics, Oxford Univ. Press, Oxford, 1999

[AW63] Araki, H., and E. J. Woods, Representations of the canonical commutation relations describing a nonrelativistic infinite free Bose gas, J. Math. Phys. 4 (1963), 637-662

[AW68] - A classification of factors, Publ. RIMS, Kyoto Univ. Ser. A 3 (1968), 51-130

[AZ05] Araki, H., and L. Zsidó, Extension of the structure theorem of Borchers and its application to half-sided modular inclusions, Rev. Math. Phys. 17:5 (2005), 491-543

[Ba64] Bargmann, V., Note on Wigner's Theorem on Symmetry Operations, Journal of Mathematical Physics 5:7 (1964), 862-868

[BJL02] Baumgärtel, H., Jurke, M., and F. Lledo, Twisted duality of the CAR-algebra, J. Math. Physics 43:8 (2002), 4158-4179

[BCL10] Bertozzini, P., R. Conti, and W. Lewkeeratiyutkul, Modular theory, Noncommutative Geometry and Quantum Gravity, in "Symmetry, Integrability and Geometry: Methods and Applications," SIGMA 6 (2010), 067; 47pp

[Be96] Bertram, W., Un théorème de Liouville pour les algèbres de Jordan, Bull. Soc. Math. France 124:2 (1996), 299-327

[BW76] Bisognano, J. J., and E. H. Wichmann, On the duality condition for quantum fields, J. Math. Phys. 17 (1976), 303-321 
[Bla06] Blackadar, B., "Operator Algebras," Encyclopaedia of Mathematical Sciences Vol. 122, Springer-Verlag, Berlin, 2006.

[Bo68] Borchers, H.-J., On the converse of the Reeh-Schlieder theorem, Comm. Math. Phys. 10 (1968), 269-273

[Bo92] - The CPT-Theorem in two-dimensional theories of local observables, Comm. Math. Phys. 143 (1992), 315-332

[Bo95] - On the use of modular groups in quantum field theory, Ann. Ins. H. Poincaré 63:4 (1995), 331-382

[Bo96] - Half-sided modular inclusion and the construction of the Poincaré group, Commun. Math. Phys. 179 (1996), 703-723

[Bo97] - On the lattice of subalgebras associated with the principle of half-sided modular inclusion, Lett. Math. Phys. 40:4 (1997), 371-390

[Bo98] - Half-sided translations and the type of von Neumann algebras, Lett. Math. Phys. 44 (1998), 283-290

[Bo00] - On revolutionizing quantum field theory with Tomita's modular theory, J. Math. Phys. 41 (2000), 3604-3673; extended version with complete proofs available under ftp://ftp.theorie.physik.uni-goettingen.de/pub/papers/lqp/99/04/esi773.ps.gz

[BR87] Bratteli, O., and D. W. Robinson, "Operator Algebras and Quantum Statistical Mechanics I," 2nd ed., Texts and Monographs in Physics, Springer-Verlag, 1987

[BR96] —, "Operator Algebras and Quantum Statistical Mechanics II," 2nd ed., Texts and Monographs in Physics, Springer-Verlag, 1996

[BtD85] Bröcker, T., and T. tom Dieck, "Representations of Compact Lie Groups," Springer, Graduate Texts in Mathematics 98, 1985

[BGL93] Brunetti, R., Guido, D., and R. Longo, Modular structure and duality in conformal quantum field theory, Comm. Math. Phys. 156 (1993), 210-219

[BGL02] —, Modular localization and Wigner particles, Rev. Math. Phys. 14 (2002), 759-785 [Bu78] Buchholz, D., On the structure of local quantum fields with non-trivial interaction, in "Proceedings of the Int. Conf. on Operator Algebras, Ideals and their Application in Theoretical Physics," Baumgärtel, Lassner, Pietsch, Uhlmann (eds.), 146-153; Teubner Verlagsgesellschaft, Leipzig

[BDFS00] Buchholz, D., O. Dreyer, M. Florig, and S. J. Summers, Geometric modular action and spacetime symmetry groups, Rev. Math. Phys. 12:4 (2000), 475-560

[BLS11] Buchholz, D., Lechner, G., and S. J. Summers, Warped convolutions, Rieffel deformations and the construction of quantum field theories, Comm. Math. Phys. 304:1 (2011), 95-123

[BS93] Buchholz, D., and S. J. Summers, An algebraic characterization of vacuum states in Minkowski space, Comm. Math. Phys. 155:3 (1993), 449-458

[Co73] Connes, A., Une classification des facteurs de type III, Annales scientifiques de lÉ.N.S. 4iéme série 6:2 (1973), 133-252

[Co74] - Caractérisation des espaces vectoriels ordonnés sous-jacents aux algèbres de von Neumann, Annales de l'institut Fourier 24:4 (1974), 121-155

[CR94] Connes, A., and C. Rovelli, Von Neumann Algebra Automorphisms and TimeThermodynamics Relation in General Covariant Quantum Theories, Class. Quant. Grav. 11 (1994), 2899-2918

[vD71] van Daele, Quasi-equivalence of quasi-free states on the Weyl algebra, Commun. Math. Phys. 21 (1971), 171-191

[EO73] Eckmann, J.-P., and K. Osterwalder, An application of Tomita's theory of modular 
[FK94] Faraut, J., and A. Koranyi, "Analysis on symmetric cones," Oxford Math. Monographs, Oxford University Press, 1994

[FG89] Figliolini, F., and D. Guido, The Tomita operator for the free scalar field, Ann. Inst. H. Poincaré, Phys. Théor. 51 (1989), 419-435

[F198] Florig, M., On Borchers' Theorem, Lett. Math. Phys. 46 (1998), 289-293

[Fo83] Foit, J., Abstract twisted duality for quantum free Fermi fields, Publ. Res. Inst. Math. Sci. 19:2 (1983), 729-741

[Gu11] Guido, D., Modular theory for the von Neumann algebra of local quantum physics, Contemp. Math. 534 (2011), 97-120

[GL95] Guido, D., and R. Longo, An algebraic spin and statistics theorem, Commun. Math. Physics 172 (1995), 517-533

[GLW98] Guido, D., Longo, R., and H.-W. Wiesbrock, Extensions of conformal nets and superselection structures, Commun. Math. Physics 192 (1998), 217-244

[Ha96] Haag, R., "Local Quantum Physics. Fields, Particles, Algebras," Second edition, Texts and Monographs in Physics, Springer-Verlag, Berlin, 1996

[HN12] Hilgert, J., and K.-H. Neeb, "Structure and Geometry of Lie Groups", Springer, 2012

[HÓ96] Hilgert, J., and G. Ólafsson. Causal Symmetric Spaces, Geometry and Harmonic Analysis. Perspectives in Mathematics 18, Academic Press, 1996

[HL82] Hislop, P., and R. Longo, Modular structure of the local observables associated with the free massless scalar field theory, Comm. Math. Phys. 84:1 (1982), 71-85

[JM17] Jäkel, C., and J. Mund, The Haag-Kastler axioms for the $P(\varphi)_{2}$ model on the de Sitter space, arXiv:math-ph:1701.08231

[KW01] Kähler, R., and H.-W. Wiesbrock Modular theory and the reconstruction of fourdimensional quantum field theories, J. Math. Phys. 42:1 (2001), 74-86

[Ka97] Kaup, W., On real Cartan factors, manuscripta math. 92 (1997), 191-222

[Ke96] Keyl, M., Causal spaces, causal complements and their relations to quantum field theory, Rev. Math. Phys. 8:2 (1996), 229-270

[Ke98] - How to describe the space-time structure with nets of $C^{*}$-algebras, Internat. J. of Theoretical Physics 37:1 (1998), 375-385

[Kl11] Klotz, M., Banach Symmetric Spaces, Preprint, arXiv:math.DG:0911.2089

[KS05] Krötz, B., and R. J. Stanton, Holomorphic extension of representations: (II) geometry and harmonic analysis, Geom. Funct. Anal. 15 (2005), 190-245

[Le15] Lechner, G., Algebraic constructive quantum field theory: Integrable models and deformation techniques, Preprint, arXiv:math.ph:1503.03822

[LL14] Lechner, G., and R. Longo, Localization in Nets of Standard Spaces, Comm. Math. Phys., to appear

[Lle09] Lledó, F., Modular theory by example, Preprint, arXiv:math.OA:0901.1004v1

[Lo82] Longo, R., Algebraic and modular structure of von Neumann algebras of physics, in "Operator algebras and applications, Part 2 (Kingston, Ont., 1980)," pp. 551-566, Proc. Sympos. Pure Math., 38, Amer. Math. Soc., Providence, R.I., 1982

[Lo08] - Real Hilbert subspaces, modular theory, SL(2, R) and CFT in "Von Neumann Algebras in Sibiu", 33-91, Theta Ser. Adv. Math. 10, Theta, Bucharest,

[LW11] Longo, R., and E. Witten, An algebraic construction of boundary quantum field theory, Comm. Math. Phys. 303:1 (2011), 213-232

[Lo69] Loos, O., "Symmetric spaces I: General theory," W. A. Benjamin, Inc., New York, 
Amsterdam, 1969

[Mo17] Morinelli, V., The Bisognano-Wichmann property on nets of standard subspaces, some sufficient conditions, arXiv:math-ph:1703.06831

[Mu01] Mund, J., The Bisognano-Wichmann theorem for massive theories, Ann. Henri Poincaré 2:5 (2001), 907-926

[MSY06] Mund, J., Schroer, B., and J. Yngvason, String-localized quantum fields and modular localization, Comm. Math. Phys. 268:3 (2006), 621-672

The Bisognano-Wichmann theorem for massive theories, Ann. Henri Poincaré 2:5 (2001), 907-926

[Ne00] Neeb, K.-H., "Holomorphy and Convexity in Lie Theory," Expositions in Mathematics 28, de Gruyter Verlag, Berlin, 2000

[NÓ14] Neeb, K.-H., G. Ólafsson, Reflection positivity and conformal symmetry, J. Funct. Anal. 266 (2014), 2174-2224

[NO15] - Reflection positive one-parameter groups and dilations, Complex Analysis and Operator Theory 9:3 (2015), 653-721

[NÓ16] - KMS conditions, standard subspaces and reflection positivity on the circle group, arXiv:math-ph:1611.00080; submitted

[NÓ17] - Antiunitary representations of hermitian groups, in preparation

[NÓ17b] - Reflection positivity on spheres and hyperboloids, in preparation

[OM16] Oppio, M., and V. Moretti, Quantum theory in real Hilbert space: How the complex Hilbert space structure emerges from Poincaré symmetry, Preprint arXiv:mathph:1611.09029v1 28 Nov 2016

[Ra17] Raasakka, M., Spacetime-free approach to quantum theory and effective spacetime structure, arXiv:1605.03942v2 [gr-qc] 24 Jan 2017

[RS73] Reed, S., and B. Simon, "Methods of Modern Mathematical Physics I: Functional Analysis," Academic Press, New York, 1973

[RS61] Reeh, H., and S. Schlieder, Bemerkungen zur Unitäräquivalenz von Lorentzinvarianten Feldern, Nuovo Cimento 22 (1961), 1051-1068

[Sa97] Salehi, H., Problems of dynamics in generally covariant quantum field theory, Internat. J. Theoret. Phys. 36:1 (1997), 143-155

[Sch97] Schroer, B., Wigner representation theory of the Poincaré group, localization, statistics and the S-matrix, Nuclear Phys. B 499-3 (1997), 519-546

[Sch06] - Positivity and Integrability (Mathematical Physics at the FU-Berlin), Preprint, arXiv:hep-th/0603118

[Sch09] - Localization and the interface between quantum mechanics, quantum field theory and quantum gravity II. The search of the interface between QFT and QG, Stud. Hist. Philos. Sci. B Stud. Hist. Philos. Modern Phys. 41:4 (2010), 293-308

[SW00] Schroer, B., and H.-W. Wiesbrock, Modular theory and geometry, Rev. Math. Phys. 12:1 (2000), 139-158

[So10] Solveen, C., The Bisognano-Wichmann Theorem and nets on $\mathbb{R}^{4}$, manuscript from http://math.mit.edu/ eep/CFTworkshop

[StVa02] Stalder, Y., and A. Valette, Le lemme de Schur pour les représentations orthogonales, Expo. Math. 20 (2002), 279-285

[Su05] Summers, S., Tomita-Takesaki modular theory, arXiv:0511.034v1 [math-ph] 9 Nov 2005

[SW03] Summers, S., and R. K. White, On deriving space-time from quantum observables and states, Commun. Math. Phys. 237 (2003), 203-220 
[Ta12] Tanimoto, Y., Construction of wedge local nets of observables through Longo-Witten endomorphisms, Comm. Math. Phys. 314:2 (2012), 443-469

[Tr97] Trebels, S., Über die geometrische Wirkung modularer Automorphismen, PhD Thesis, Univ. Göttingen, 1997

[Va85] Varadarajan, V. S., "Geometry of Quantum Theory," Springer Verlag, 1985

[Wa98] Wassermann, A., Operator algebras and conformal field theory. III. Fusion of positive energy representations of $L S U(N)$ using bounded operators, Invent. Math. 133 (1998), 467-538

[Wi92] Wiesbrock, H.-W., A comment on a recent work of Borchers, Lett. Math. Phys. 25 (1992), 157-159

[Wi93] -, Half-sided modular inclusions of von Neumann algebras, Commun. Math. Phys. 157 (1993), 83-92

[Wi93b] - Conformal quantum field theory and half-sided modular inclusions of von Neumann algebras, Comm. Math. Phys. 158:3 (1993), 537-543

[Wi93c] - Symmetries and half-sided modular inclusions of von Neumann algebras, Lett. Math. Phys. 28:2 (1993), 107-114

[Wi97] - Half-sided modular inclusions of von Neumann algebras, Erratum, Commun. Math. Phys. 184 (1997), 683-685

[Wi97b] - Symmetries and modular intersections of von Neumann algebras, Lett. Math. Phys. 39:2 (1997), 203-212

[Wi98] - Modular intersections of von Neumann algebras in quantum field theory, Comm. Math. Phys. 193:2 (1998), 269-285

[Wig59] Wigner, E., "Group Theory and its Applications to the Quantum Mechanics of Atomic Spectra," Academic Presse, 1959 